\title{
The Effect of 2D and 3D Menus on Memory Retention in User Interface Design
}

\author{
A Thesis \\ presented to \\ the Faculty of California Polytechnic State University, \\ San Luis Obispo
}

\author{
In Partial Fulfillment \\ of the Requirements for the Degree \\ Master of Science in Industrial Engineering
}

by

Angela M. Muscat

June, 2013 
(C) 2013

Angela M. Muscat

ALL RIGHTS RESERVED

Page ii 
COMMITTEE MEMBERSHIP

TITLE:

AUTHOR:

DATE SUBMITTED:

COMMITTEE CHAIR:

COMMITTEE MEMBER:

COMMITTEE MEMBER:
The Effect of 2D and 3D Menus on Memory Retention in User Interface Design

Angela M. Muscat

June, 2013

Dr. Reza Pouraghabagher, Ph.D.

Industrial and Manufacturing Engineering Department

California Polytechnic State Univ., San Luis Obispo

Dr. Lizabeth Schlemer, Ph.D.

Industrial and Manufacturing Engineering

Department

California Polytechnic State Univ., San Luis Obispo

Dr. Roya Javadpour, Ph.D.

Industrial and Manufacturing Engineering

Department

California Polytechnic State Univ., San Luis Obispo 


\begin{abstract}
The Effect of 2D and 3D Menus on Memory Retention in User Interface Design Angela M. Muscat
\end{abstract}

The increasing use of 3D user interface elements, particularly 3D menus, demonstrates the need to expand research in the field of Human Computer Interaction (HCI) as it pertains to 3D user interfaces. The results of this thesis contribute to the understanding of the cognitive impacts of using 3D menus. Multiple application areas for 3D menus have been identified where memory retention is a critical success factor, but little research has been done in the area of memory retention for 3D menus. The purpose of this thesis is to investigate if the use of 3D carousel menus increases retention of information over $2 \mathrm{D}$ menus and if is there a gender effect with these results. A three factor split-plot (one-between subject factor and two-within subject factors) experiment was designed to test if menu dimension, content type, and gender are significant factors in memory retention and to determine if there are any interactions between these factors. The results of the experiment revealed that dimension and gender are not significant factors in the retention of information and none of the interactions of dimension (2D vs. 3D), gender, and content were significant. Several subjects' questionnaire responses demonstrated that the menu dimension they perceived to better aid retention was 3D; however these results were not statistically significant. While these results showed that within the boundaries chosen the use of a 3D menu neither promotes nor degrades memory retention, there are still a number of questions that need to be answered regarding the use of 3D menus and their effect on other cognitive processes. 
Keywords: 3D Carousel, 3D Menus, 2D Menus, Visual Displays, Memory Retention, User Interface Design, Human Computer Interaction 


\section{ACKNOWLEDGMENTS}

I would like to thank my advisor, Dr. Pouraghabagher, for his guidance throughout this process. His knowledge of HCI was an invaluable resource as I pursued research in the field. I would also like to thank Dr. Schlemer and Dr. Javadpour for their feedback and support as my thesis committee members. Finally, a large thank-you goes to my friends and family who have encouraged me to pursue my academic goals and supported me throughout my time at Cal Poly. 


\section{TABLE OF CONTENTS}

LIST OF TABLES

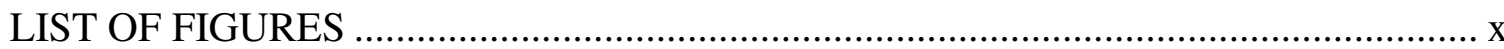

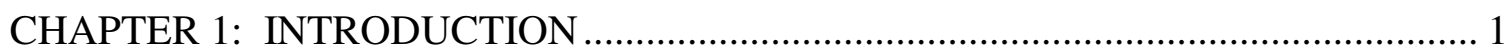

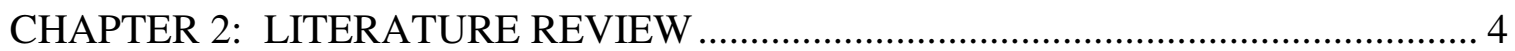

2.1 Navigation Menus in Interface Design and the Transition to 3D ................. 4

2.1.1 Purpose of Navigation Menus in GUIs .............................................. 4

2.1.2 Transition to Animated and 3D Menus.............................................. 4

2.1.3 3D as a Natural Interaction Style …………………………........ 5

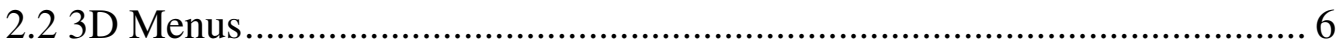

2.2.1 3D Carousel Design ...................................................................... 6

2.2.2 3D Carousel Animation .............................................................. 7

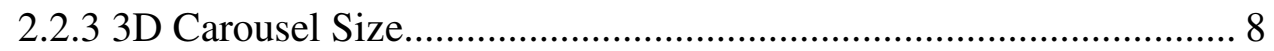

2.2.4 3D Carousel Data Types and Design Characteristics ........................ 8

2.3 Research on Advantages and Disadvantages of 3D Carousels ...................... 9

2.3.1 Menu Depth and Breadth ............................................................ 9

2.3.2 Task Efficiency ……………………………………………..... 10

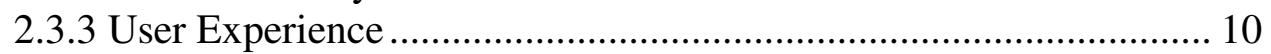

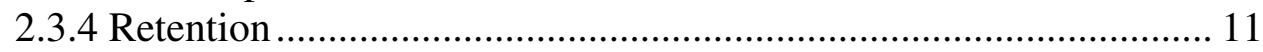

2.4 The Importance of Retention and 3D Carousels......................................... 12

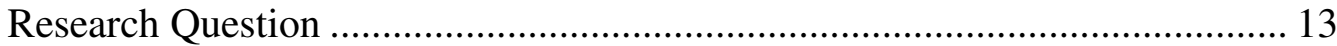

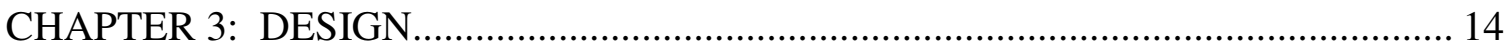

3.1 Design of the 2D and 3D Carousels........................................................ 14

3.2 Content and Assessment ..................................................................... 15

3.2.1 Abstract Objects......................................................................... 16

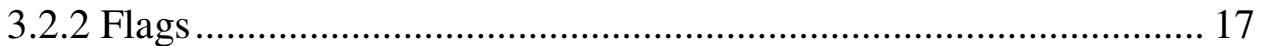

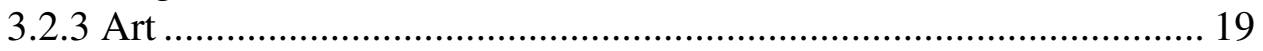

3.2.4 Considerations for Cognitive Load ................................................. 21

3.3 Technical Development and Implementation ........................................... 22

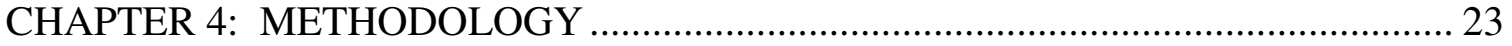

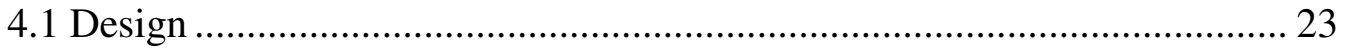

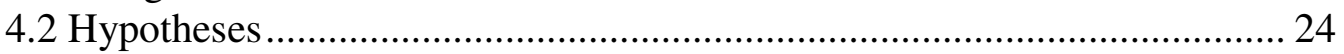

4.3.1 Independent Variables ............................................................... 24

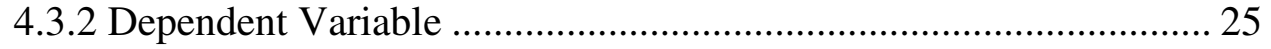

4.3.3 Controlled Variables ………………………………………….... 25

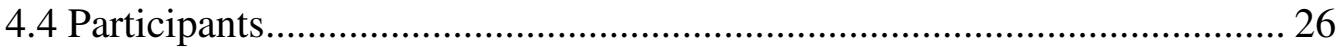

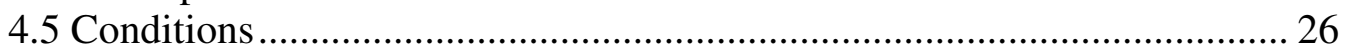

4.5.1 Randomization of the Image Sets .................................................. 30

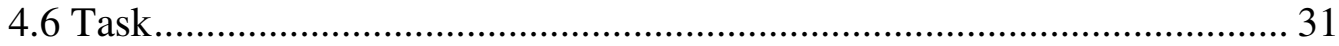

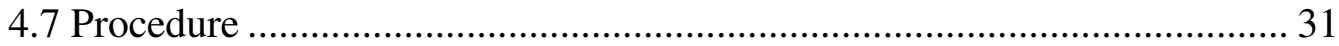

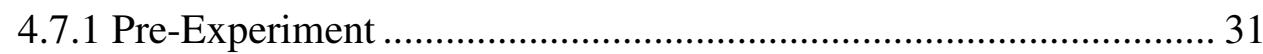

4.7.2 Training Trials and Experiment ..................................................... 32

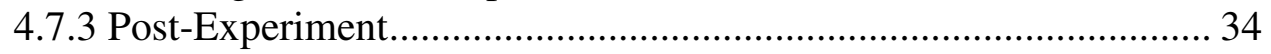

4.8 Experimental Setting and Apparatus ………………………………...... 35

Page vii 


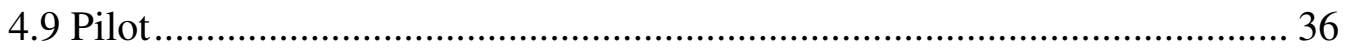

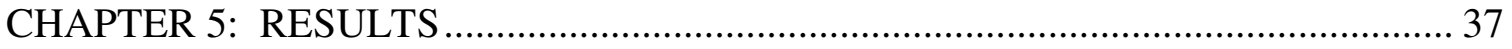

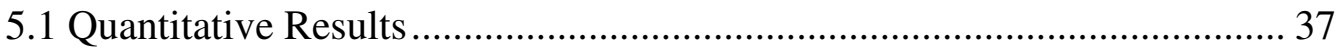

5.1.1 Raw Data and Descriptive Statistics ....................................... 37

5.1.2 Test of Normality ............................................................. 38

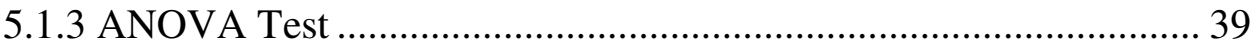

5.1.4 Interaction Plots ........................................................................ 40

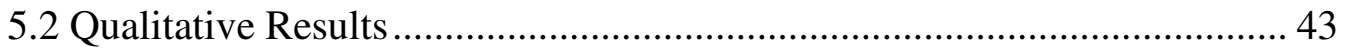

5.2.1 Raw Data and Descriptive Statistics ......................................... 43

5.2.2 Test of Normality ............................................................ 45

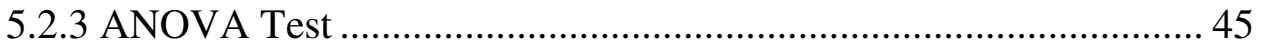

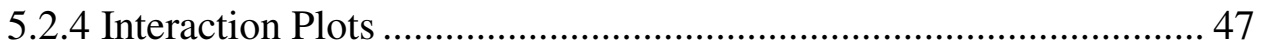

5.2.5 Subject Comments ............................................................ 50

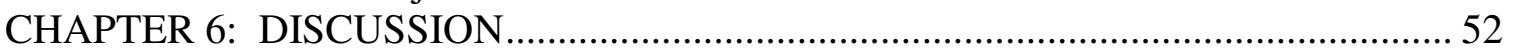

6.1 Quantitative Results ....................................................................... 52

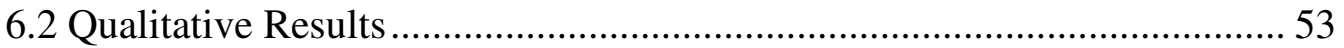

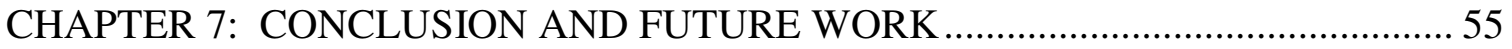

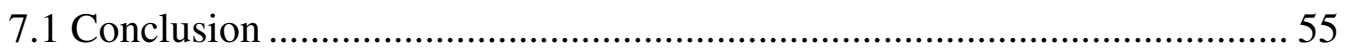

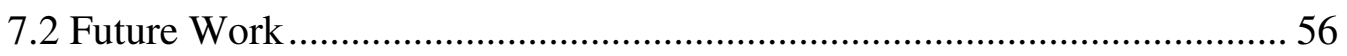

References........................................................................................ 58

APPENDIX A: Image Sets and Assessment Questions ............................... 62

APPENDIX B: Carousel Implementation and Development ...................... 126

APPENDIX C: Subject Demographics................................................... 143

APPENDIX D: Experiment Instructions ............................................. 144

APPENDIX E: Informed Consent Form .............................................. 145

APPENDIX F: Raw Quantitative Data.................................................. 146

APPENDIX G: Raw Questionnaire Data ............................................. 147

APPENDIX H: Subjects' Comments .................................................... 148 


\section{LIST OF TABLES}

Table

Page

Table 1 Factors and Levels $\quad 23$

Table 2 Experimental Design $\quad 23$

Table 3 Hypotheses $\quad 24$

Table 4 Nature of Question for Each Content Type 31

Table 5 Descriptive Statistics for Quantitative Response Variable 37

Table 6 ANOVA Factors and Levels $\quad 39$

Table 7 Random Effect Variance Component Estimates 39

Table 8 Fixed Effect Test Results $\quad 40$

Table 9 Descriptive Statistics for Questionnaire Response Data 44

Table 10 ANOVA Factors and Levels 46

Table 11 Random Effect Variance Component Estimates 46

Table 12 Fixed Effect Test Results $\quad 47$

Table 13 Subject Demographics $\quad 143$

Table 14 Raw Quantitative Data 146

Table 15 Raw Questionnaire Data 147

Table 16 Subjects' Comments from Questionnaire 148 


\section{LIST OF FIGURES}

Figure

Page

Figure 1 Example of a 3D Menu, Apple's Cover Flow [4] ................................. 1

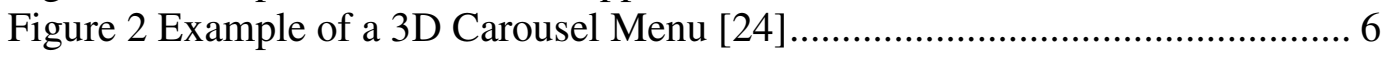

Figure 3 Basic 3D Carousel Model [25] ........................................................ 7

Figure 4 Samples of 2D and 3D Carousel Design ........................................... 14

Figure 5 Abstract Object Sample Question ........................................................ 16

Figure 6 Flag Sample Question - Country Name................................................ 18

Figure 7 Flag Sample Question - Color Scheme ............................................... 18

Figure 8 Flag Sample Question - Object or Shape ............................................. 19

Figure 9 Art Sample Question - Painting Name …............................................ 20

Figure 10 Art Sample Question - Subject of Painting ....................................... 20

Figure 11 Art Sample Question - Painting Setting .......................................... 20

Figure 12 Art Sample Question - Number of People in Painting ........................ 21

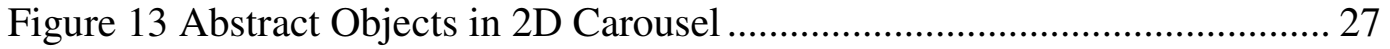

Figure 14 Abstract Objects in 3D Carousel …................................................ 28

Figure 15 Flags in 2D Carousel ..................................................................... 28

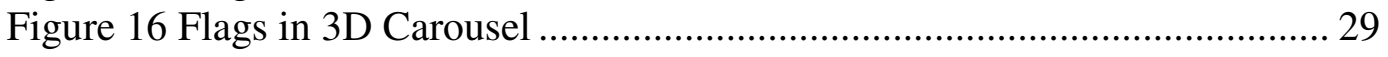

Figure 17 Art in 2D Carousel..................................................................... 29

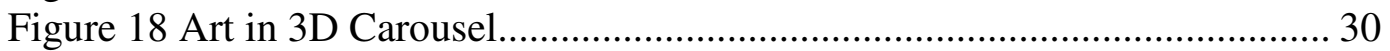

Figure 19 Screen Shot of Subject Information Form........................................ 32

Figure 20 Screen Shot of Tables ..................................................................... 33

Figure 21 Sample of Carousel Ranking Question........................................... 34

Figure 22 Final Questionnaire Question ........................................................ 35

Figure 23 Histogram of Quantitative Responses ............................................ 38

Figure 24 Gender vs. Dimension Interaction Plot ............................................ 40

Figure 25 Gender vs. Content Interaction Plot ............................................... 41

Figure 26 Dimension vs. Content Interaction Plot......................................... 42

Figure 27 Gender vs. Dimension vs. Content .................................................... 42

Figure 28 Histogram of Questionnaire Responses............................................ 45

Figure 29 Gender vs. Dimension Interaction Plot ............................................. 48

Figure 30 Gender vs. Content Interaction Plot ............................................... 48

Figure 31 Dimension vs. Content Interaction Plot.......................................... 49

Figure 32 Gender vs. Content vs. Dimension Interaction Plot .......................... 50

Figure 33 Summary of Comments from Questionnaire.................................... 51 


\section{CHAPTER 1: INTRODUCTION}

The transition of user interface design to three dimensional (3D) displays and virtual reality environments is a user experience shift that is sweeping across many technological platforms today, including computers, cell phones, and tablet devices. Since the first arrival of the Graphical User Interface (GUI) around 1980, Human Computer Interaction (HCI), specifically user interface design, has become a very important area of research. The field of HCI encompasses a variety of disciplines including perception, cognition, graphic design, and human factors. For years the conventional user interface presentations were two dimensional (2D) by nature which makes three dimensional user interface design a relatively new research area within HCI. Figure 1 shows an example of a common 3D GUI, the Cover Flow, which was introduced by Apple in 2006. Three dimensional user interface design is still a growing field with vast possibilities, but many research questions regarding the HCI with 3D remain unanswered.

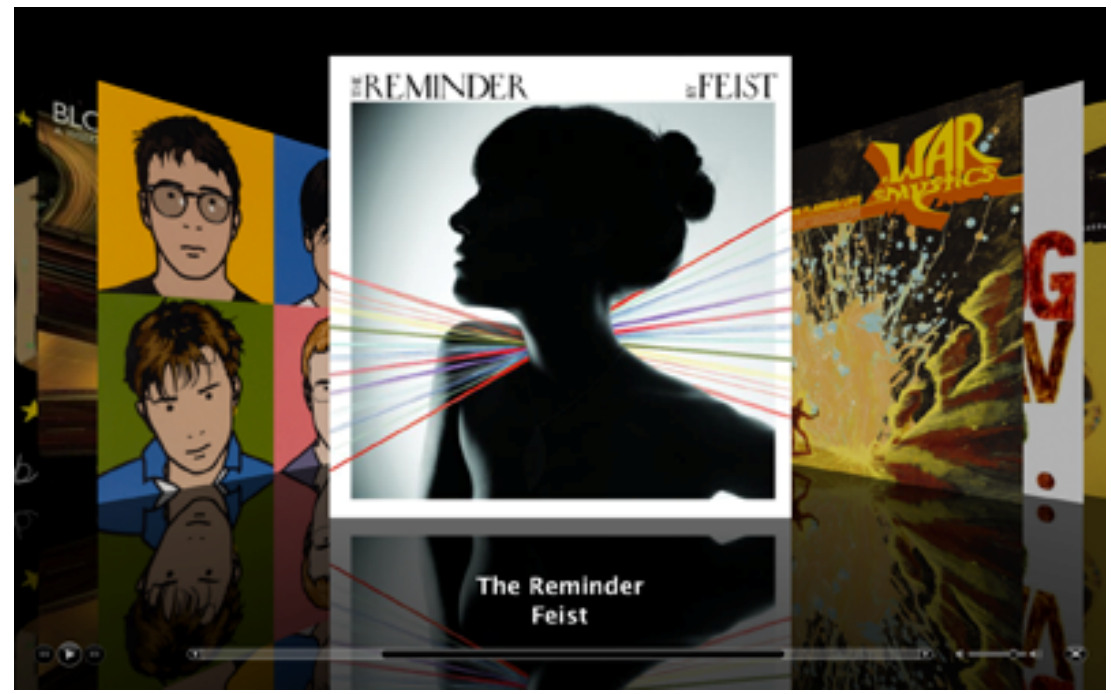

Figure 1 - Example of a 3D Menu, Apple's Cover Flow [4] 
Although the capability to generate complex 3D environments has been possible for quite some time, a primary example being video games, the platform support necessary to easily generate these images on the web has only recently been developed. These recent advances have produced an increase in web features such as three dimensional menus, which are the focus of this study. While the application potential for 3D menus is very large, some specific applications have been identified by researchers as ones where retention of information is a primary criterion. Examples include tutorials, training, e-learning, and e-commerce sites [18].

Initial research with 3D menus has had positive user experience feedback, concluding that 3D menus are a fun, interactive technique [11]. Three dimensional menus have generally been well received for their intuitive aesthetic design [25]. While aesthetics is one factor for designers and developers of software to consider, there are several other critical aspects, such as human cognition, that may have a significant impact on the user interface. Effective user interface design takes into account the cognitive and perceptual capabilities of humans and considers how the design impacts the mental workload of the user. The way in which user interface design influences cognitive load is particularly important in those applications where retention is a critical success factor. Research has yet to explore the effects, if any, on memory retention when images are displayed in a 3D menu in comparison to a $2 \mathrm{D}$ menu.

The growing presence of 3D user interface elements, specifically 3D menus, demonstrates the need to expand the research in the $\mathrm{HCI}$ field as it pertains to 3D user interfaces. Designers of user interfaces are inherently concerned with making design decisions that support human cognitive abilities while decreasing their memory 
workload. Since there are many areas of user interface design that relate to applications where memory retention is a concern, exploring the potential benefits that displaying items in a third dimension has on memory retention is worthwhile. The objective of this research is to investigate if use of $3 \mathrm{D}$ menus in user interface design has a positive impact on retention over $2 \mathrm{D}$ displays. 


\section{CHAPTER 2: LITERATURE REVIEW}

This section provides background information into the topic of 3D navigational menus and explores the current research in the field. Background information is provided to establish the importance of navigation menus in user interface (UI) design and define $3 \mathrm{D}$ models in this application. Some of the recent literature in regard to 3D menus is summarized and a further avenue for research is identified, which is the focus of this thesis.

2.1 Navigation Menus in Interface Design and the Transition to 3D

\subsubsection{Purpose of Navigation Menus in GUIs}

GUIs frequently use menu-driven interfaces. Generally, a menu is defined as a set of options displayed on a screen in which the selection of one or more options results in a change in the state of the interface [17].GUIs employ menus as a means of allowing users to access system functionality and navigation; a menu is a primary aspect of a user's experience with the GUI for any information system [16]. Through appropriate selections of navigational pathways, users are able to effectively retrieve information [7]. Since a menu gives a user access to information and system functionality while also dictating the organizational structure of the system, the menu is a key aspect of a user's experience with a GUI.

\subsubsection{Transition to Animated and 3D Menus}

The GUIs predominantly seen in computer applications are fundamentally 2D; however, the current systems are capable of interactive 3D graphics, computer games being a common example [18]. Recent advances in various programming platforms have made it easier for developers and designers to add sleek animations to navigation design 
[9]. These dynamic or active animations in navigation design often include 3D translations of items that were previously only represented in 2D. Traditionally in UI design, programmers work with a two dimensional, $\mathrm{XY}$ plane in which all items have a height and width and are moved around a screen horizontally and vertically. Translating objects into 3D means that a third plane, the $\mathrm{Z}$ plane, is added to create an element of depth for each object, as if objects are moving toward and away from the user [6].

Researchers in this field have noted that 3D space is more powerful in terms of design capability than $2 \mathrm{D}$ space because $2 \mathrm{D}$ space is contained in $3 \mathrm{D}$ space [11]. Designers and developers have been using this 3D effect to make the screen's space appear more dense in the sense that a screen can hold more objects, which a user can now animate [20]. Animation, although often associated with appearance and the "look and feel" of a GUI, has a significant impact on the users' interaction with the menu. There are considerably more possibilities for animation in 3D space compared to $2 \mathrm{D}$; some of the available techniques are: zooming-in, turning, expanding, rotating, and collapsing [5].

\subsubsection{D as a Natural Interaction Style}

As the enthusiasm for pushing $2 \mathrm{D}$ objects into the third dimension grows, there is an interest in studying the impact that an additional dimension has on the user's interaction with the interface. Although 3D seems to provide a new interaction technique for users to learn and familiarize themselves with, many researchers have identified 3D as a natural transition for GUI design that is more intuitive with users' spatial capabilities. Researchers see 3D as a better way of organizing data that people are more familiar with [25]. From living in a 3D world where objects are organized spatially, people are innately proficient at remembering spatial relationships [1]. Further research has shown that the 
use of spatial relationships in $3 \mathrm{D}$ environments is advantageous because this provides a more intuitive style of interaction that users perceive with a lower cognitive load [11] [15]. Users' preattentive abilities based on the depth cues provided by 3D allow them to understand the spatial relationships between the objects on an interface subconsciously [21].

\subsection{D Menus}

Common types of 3D menus for computers are cone tree, data mountain, collapsible cylindrical tree, 3D carousel, and revolving stage. The 3D carousel has quickly become a common feature on websites [24]. Figure 2 shows an example of a 3D carousel menu. Adaptations of the 3D carousel are prevalent in many computer applications and are common features in video game display menus [25]. The 3D carousel menu has also been identified as a menu style that is potentially effective for mobile devices due to their ability to present more items than 2D menus, thus enhancing the usability of devices with small screen sizes [13].

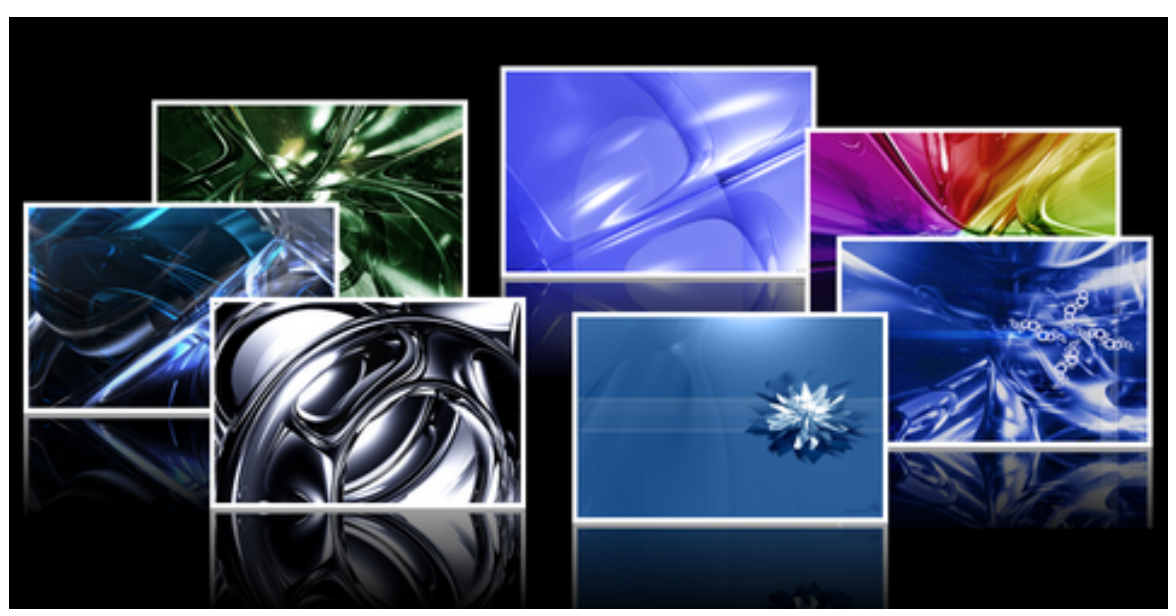

Figure 2 - Example of a 3D Carousel Menu [8] 


\subsubsection{D Carousel Design}

A 3D carousel menu is a 3D menu in which objects, text or images, are presented on a ring. The study "Designing a Generalized 3D Carousel View" defines the standard carousel as:

A 3D object with its own coordinate system (Figure 3). The base of it is a circular plinth. It lies in the XZ plane (which we call "plinth's plane"), its center being the $(0,0,0)$ point. We will refer to the $Y$ axis as the carousel's central axis. On (or above) the plinth's plane lie the "bins", each in the same distance $\boldsymbol{R}$ from the central axis. We will call $\boldsymbol{R}$ the "carousel's radius". Each bin represents one object from the dataset... The standard implementation of the carousel evenly distributes the bins around the circumference of the plinth. [25]

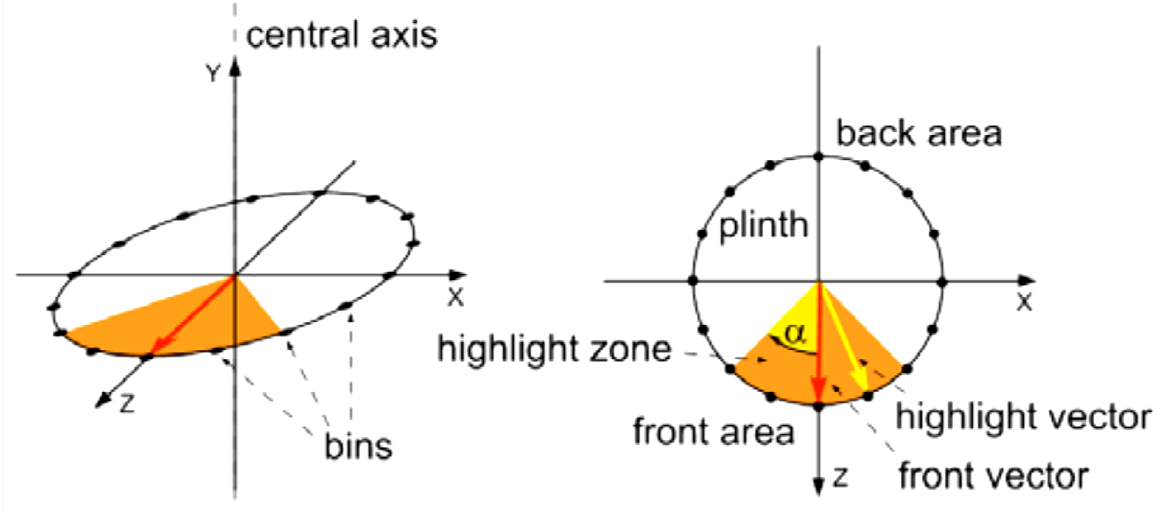

Figure 3 - Basic 3D Carousel Model [25]

\subsubsection{D Carousel Animation}

Animation is another key component to the design and user interaction of a 3D

carousel. There are multiple types of animation that can be employed including the rotation of the ring and zooming in and out on the bins. Rotation of the ring is caused by the user's interaction with the carousel itself or with controls that move the carousel; an exception to this is a freely spinning carousel. Free spinning carousels move without user 
interaction and stop spinning once the user interacts with the carousel. Feedback from

users in previous studies indicated that with a fast rotating carousel, users can sometimes experience vertigo [25].

\subsubsection{D Carousel Size}

Carousels are typically used for scenarios where users select an option from a small set of items (generally no larger than 12); however adaptations of 3D carousels have also been used in applications with larger numbers of options or items, such as file or image browsing through a library [11][25]. Carousels can be expanded to hold a larger number of items by either hiding or compressing the bins that are in the back area of the ring; this method can support any number of bins.

\subsubsection{D Carousel Data Types and Design Characteristics}

The 3D carousel menu is suitable for displaying a variety of data types. These types include:

- 3D-Objects

- 3D-Objects and text

- Text entries

- Images

- Images and text

The images and text combined data type is most appropriate and most common for 3D carousels [5] [25]. A design characteristic of 3D menus often associated with user interaction is feedback highlighting. Feedback highlighting is the method by which the interface indicates to the user which item on the carousel is the selected or target item. 
Common methods for feedback highlighting in 3D menus are changing the color, brightness, geometry, or size of the target item [5].

\subsection{Research on Advantages and Disadvantages of 3D Carousels}

Current research in this field has explored a variety of effects that the 3D carousel has on user interaction. Some advantages to 3D menus that have been identified include their breadth capability and positive user experience. Although 3D navigation has been referenced as a natural interaction style that is intuitive for users, there are also areas, such as task efficiency, where 2D menus are more suitable [25]. While research has begun to look at the advantages and disadvantages of 3D carousels, there are many critical areas that impact of user interface and menu design that have not been tested with 3D carousels.

\subsubsection{Menu Depth and Breadth}

One of the advantages of 3D carousel menus is the breadth capability. Menu depth and breadth are important design considerations, thus literature regarding the issue of depth versus breadth is very rich. Menu depth refers to the number of levels in the hierarchy, while breadth refers to the number of items offered in the menu [19]. User satisfaction and better performance have been associated with greater menu breadth instead of depth [9]. By making use of a third axis to display 2D objects, 3D menus allow more items to be shown, which lend itself to building broader menus than one could in 2D [2]. The ability to maximize menu breadth is an advantage of the 3D carousel menu [11]. 


\subsubsection{Task Efficiency}

Task efficiency in menu design is particularly important in applications where the main user goal is efficient completion of tasks. In order to maximize task efficiency, time and errors are minimized. Multiple comparative studies of 2D and 3D menu designs have verified that 3D menus are less suitable for environments where task efficiency is a primary concern. One study testing the information-seeking task efficiency with various 2D, 3D, and text visualization tools found that response time was slower with the 3D interfaces than with 2D and text [22]. Further research found the 3D carousel to be less efficient than a list view, and as a result recommended the 3D carousel for cases where performance efficiency is not the most critical measure [25]. An additional study testing 3D menu structures and their effect on selection tasks found that the time to complete tasks between a 2D menu and a circulatory 3D menu had similar performance results, but found 3D to be more suitable for tasks that related to browsing [18]. Although more information can be shown in 3D menus, the rate of information access can be slower which can be a disadvantage depending on the application [11].

\subsubsection{User Experience}

Research has shown through positive user experience results that 3D carousels are a fun and interactive technique. In a study comparing the 3D carousel to a similar 2D menu, users stated that the 3D carousel was easier to use and were more likely to recommend it [24]. In the same study, researchers found that the use of a 3D carousel boosted users' perceptions of interactivity and stimulated user interaction. Additional research also demonstrated that the $3 \mathrm{D}$ carousel is a preferred menu structure in terms of 
aesthetics, and users find that the animated rotation of the carousel provides a fun user experience [25]. The 3D carousel has been identified as a suitable menu structure for applications targeting younger generations [11]. 3D carousels have also been implemented frequently in mobile applications to provide users with a playful experience and an engaging way to use an application [2].

\subsubsection{Retention}

Another specific task for user interfaces is one that requires retention of information. Retention refers to the amount of knowledge that can be remembered after a given period of time [3]. Retention of information, both text and image, is an important measure of success for computer applications in many fields, including online learning, computer based training, and web design for e-commerce. Well-designed user interfaces help reduce the mental workload and promote memorability [26]. There is little to no research currently on the implications on retention when using 3D menus instead of 2D menus.

One study investigating the user interaction of a 3D carousel menu and a 2D carousel that both presented images of different guitars and played guitar riffs as the user interacted with each guitar. The study replayed the guitar riffs for each user and measured aural recognition. A significant main effect in their statistical analysis revealed that the 3D carousel degraded aural recognition memory [24]. This is the only existing study into the effects of menu dimension on retention; however the study only focused on aural recognition, a measure not often critical in user interface design. 


\subsection{The Importance of Retention and 3D Carousels}

There are many application areas where retention of information is a critical success factor, this combined with the increasing trend toward 3D displays and the lack of research done on the effects of 3D menus on retention are the inspiration for this research. Three dimensional menus have gained popularity due to their ability to display more items than $2 \mathrm{D}$ menus, intuitive nature, and the positive user experience they promote. 3D carousel menus specifically have been researched because of their growing presence in computer applications and their suitability for displaying image and text combined. The literature for 3D carousel implementations in computer applications is growing, but there are still some effects of the human computer interaction with 3D carousels that are unknown, retention being a chief example.

Researchers have outlined potential applications of 3D carousels where retention of information is a critical success factor. Specific application areas are in e-learning and e-commerce. In education or learning environments, a 3D carousel would allow students to browse through resources presented and choose their own path. In an e-commerce environment, the capabilities provided by a menu like the 3D carousel are well suited for the browsing behavior of consumers [18] [25]. There is a bright future for 3D carousel menus; however additional research needs to be done in the area of memory retention.

Many researchers have identified 3D menus as intuitive interfaces that come at a lower cognitive load to users, but little research has been done to empirically demonstrate the effects that $3 \mathrm{D}$ menus have on various cognitive processes, memory retention being a primary example. Hence this thesis will describe an experiment to compare 2D and 3D carousels measuring memory retention of text and images. 


\section{Research Question}

Does the use of 3D carousel menus increase retention of information over 2D menus and is there a gender interaction with these results?

Chapter 3 covers a designed experiment to investigate this proposed question. 


\section{CHAPTER 3: DESIGN}

The primary concern of the design of this experiment is that the dimension of the display (3D versus 2D) is the isolated variable. This was not trivial and many design considerations were taken into account. Each consideration is justified and explained in the following section.

\subsection{Design of the 2D and 3D Carousels}

For the 3D menu a 3D carousel design was selected. 3D carousels are a commonly used style of 3D menu and are suitable for displaying image and text. The 3D carousel naturally emphasizes the center image when it is tilted by presenting it above or below the images to the sides of it. In order to provide a similar emphasis in $2 \mathrm{D}$, the center image was enlarged and presented slightly higher on the screen than the image on the left and right of it. Figure 4 shows the 2D and 3D carousel designs side by side, the $2 \mathrm{D}$ carousel on the left and the $3 \mathrm{D}$ on the right.
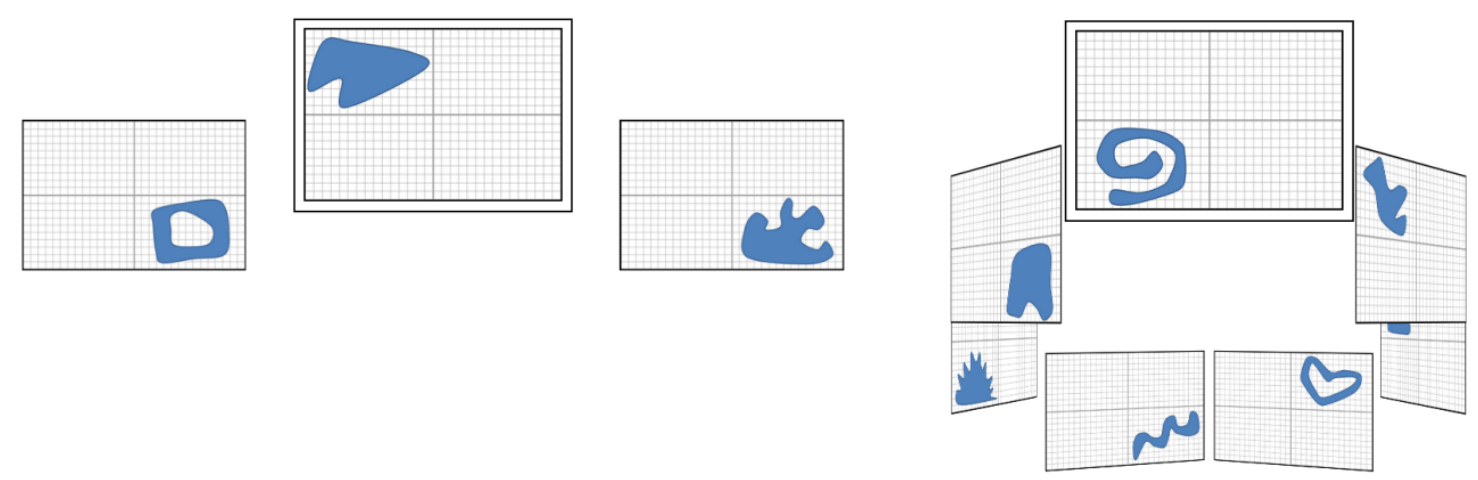

Figure 4 - Samples of 2D and 3D Carousel Design

In the 3D carousel all images in the set were somewhat visible to the user; this is a benefit of presenting images in $3 \mathrm{D}$. In the $2 \mathrm{D}$ menu, with images of the same size, presented on the same size screen, the menu can only hold three images. Presenting three images still 
gives the similar transition effect of a rotating carousel; it just appears in a more linear fashion for 2D. Another design feature implemented in both the 2D and 3D carousel is feedback highlighting. The purpose of feedback highlighting is to emphasize the center image; this is achieved by the larger size of the center image and the black border around the outside of the center image. The 2D and 3D carousels use the same type of transition, with the images moving from the right side of the screen to the left, this was selected to conform to standard design for image carousels. Moving the images from the right to the left indicates that the image to the left of the center is the previous image, and the image to the right of the center is the next image.

\subsection{Content and Assessment}

In addition to the dimension of the displays, for this research the content type and detail were varied. The three content types selected were abstract objects, flags, and art. Each content type requires a different focus or different levels of detail for the subjects to remember.

In order to assess subjects' retention of the text and images presented, questions for each type of content were generated. There were numerous questions that could be asked for each content type but some were eliminated because they could aid subjects in answering subsequent questions, could promote learning, or could be a leading question. The difficulty of the different questions for each content type was not tested prior to experimentation. This section explains the tree types of content selected and the assessment strategy for each content type. Appendix A includes the complete set of images and questions for each content type. 


\subsubsection{Abstract Objects}

For this content type, abstract objects were drawn that serve as meaningless visual symbols, unrepresentative of a specific shape or object. This content type is similar to memory tests where novel shapes without any preexisting semantic representations are presented and subjects are asked to recall which shape they saw out of a set [14].

For the abstract images, since the images do not have a name or number corresponding to them, they were placed on a standard Cartesian coordinate system in one of the four quadrants. Subjects were asked to remember which quadrant the image was in. A sample of this question type is shown in Figure 5.

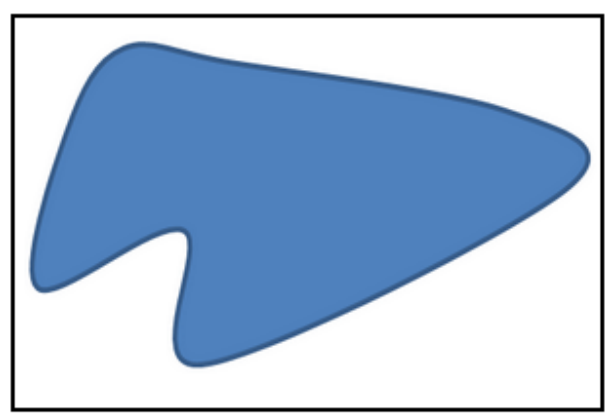

Which quadrant did the image appear in?

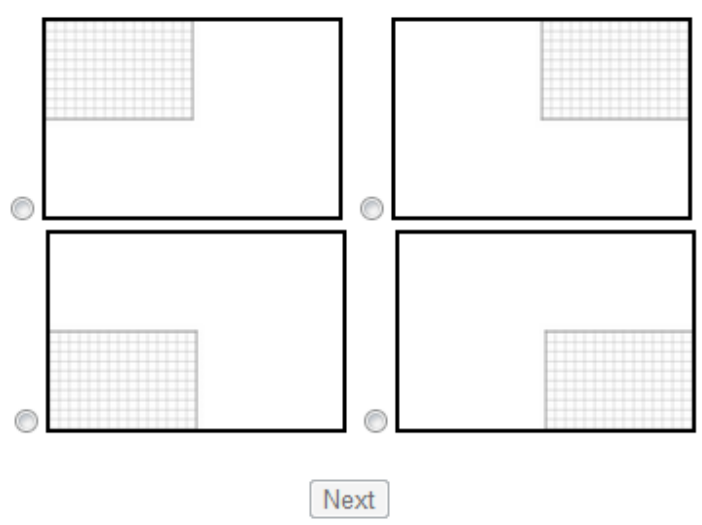

Figure 5 - Abstract Object Sample Question 


\subsubsection{Flags}

For this content type, a set of less well known country flags was selected. Country flags were selected as one of the content types for their range in level of detail. Each image has associated text (country name), and has various colors and shapes included in the design. Country flags are interesting stimuli to test retention because the country name itself does not provide any context for the subjects to remember the color scheme or design by. There are also color schemes and shapes that are common to many country flags, providing an additional level of difficulty for this content type due to the similarity of some of the images.

For the country flags there were three types of questions asked. The questions targeted different details of the flags. One question type showed subjects an image of one of the flags from the carousel and required that the subject select the country name corresponding to the flag out of a list of all the possible countries. A sample of this question type is shown in Figure 6. 


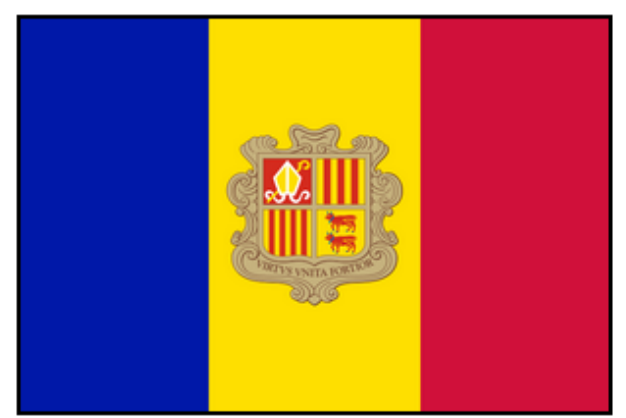

Which country does the flag above belong to?

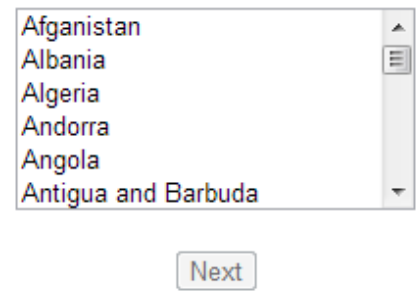

Figure 6 - Flag Sample Question - Country Name

The other two types of questions did not show a country flag, but instead asked information about the color scheme or object displayed on a flag when given a country name. Samples of these two question types are shown in Figure 7, and Figure 8.

Which one of the following color schemes is displayed on the Bolivia flag?

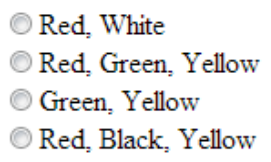

Next

Figure 7 - Flag Sample Question - Color Scheme 
Which one of the following objects or shapes is displayed on the Ukraine flag?

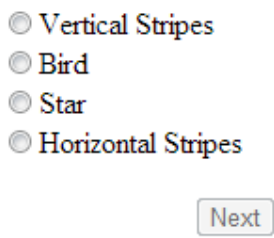

Figure 8 - Flag Sample Question - Object or Shape

While there were a variety of question types, only one question was asked per image. The type of question asked for each image was randomly assigned, but the question and answer set assigned to each image remained the same for all subjects.

\subsubsection{Art}

The Art content type is a selection of classic paintings that vary greatly in their subject matter. Each painting presented was also accompanied by the painting name. Paintings varied in their content from landscape scenes to paintings depicting complex scenarios. There were a variety of settings for the paintings and the number of people in each painting varied greatly. Art was selected as a content type because it provides rich opportunity to ask detail oriented questions and for subjects to use contextual cues to aid their retention.

There were four different types of questions asked of the art images. The types of questions asked were about the painting name, subject of the painting, setting, and number of people in the painting. A sample of the four types of questions for art images are shown in Figure 9, 10, 11, and 12. 


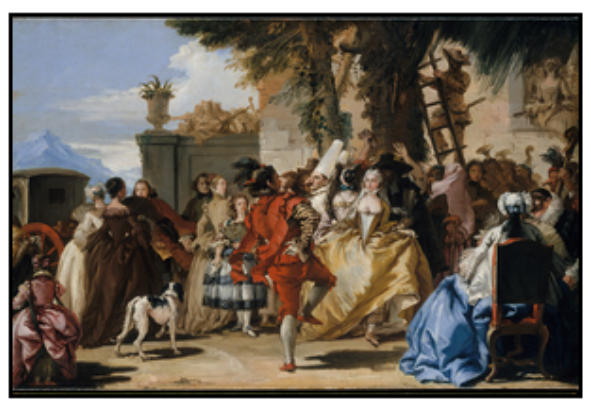

What is the name of the painting above?

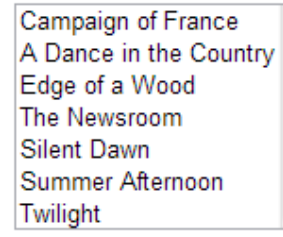

Figure 9 - Art Sample Question - Painting Name

What is the primary subject of the painting Summer Afternoon?

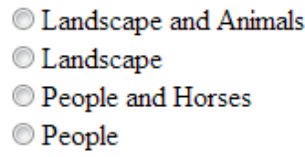

Figure 10 - Art Sample Question - Subject of Painting

What is the setting of the painting Twilight?

\author{
Outside on the Water \\ Outside on a Battle Field \\ Outside in a Field or Grassy Area \\ $\bigcirc$ Inside
}

Figure 11 - Art Sample Question - Painting Setting 
How many people are in the painting Edge of a Wood?

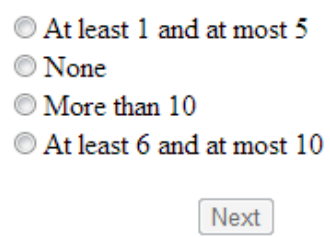

Figure 12 - Art Sample Question - Number of People in Painting

While there were a variety of question types, only one question was asked per image. The type of question asked for each image was randomly assigned, but the question and answer set assigned to each image remained the same for all subjects.

\subsubsection{Considerations for Cognitive Load}

There were two design decisions that were important considerations in terms of the cognitive load placed on the subjects. The first was the number of images presented in the carousel; the second was the length of time that they are presented. The upper limit of working memory has been defined by researchers to be approximately $7 \pm 2$ chunks of information [26]. Thus, the number of images presented in both the 2D and 3D carousel was limited to 7 images to take into account this limit.

The images need to be shown for a long enough time for subjects to observe the details, but not too long for the memory to degrade. The strength of information in working memory decays over time [26]. Various timing lengths between one and ten seconds were tested to determine the most suitable length of time to display each image for. From this testing it was determined that each image in the carousel would be shown for six seconds. 


\subsection{Technical Development and Implementation}

The carousel displays were designed by this author and developed with the assistance of a programmer using CSS3, HTML5 and JavaScript. The carousels were presented on a 15.6" laptop screen and run on the Google Chrome browser. A detailed explanation of the technical development and implementation of the carousels is found in Appendix B. 


\section{CHAPTER 4: METHODOLOGY}

\subsection{Design}

The experimental design is a three factor split-plot model of ANOVA (onebetween subject factor and two-within subject factors). The factors and levels are detailed in Table 1. Gender is the between subject factor, dimension and content are the within subject factors. There are both fixed and random variables, thus the design is a mixedeffects design.

Table 1 - Factors and Levels

\begin{tabular}{|c|c|c|}
\hline Factors & Levels & Type of Variable \\
\hline Gender & 2 & Fixed \\
\hline Dimension & 2 & Fixed \\
\hline Content & 3 & Random \\
\hline
\end{tabular}

sents the experimental design.

Table 2 presents the experimental design.

Table 2 - Experimental Design

\begin{tabular}{|l|c|c|c|c|c|c|}
\cline { 2 - 7 } \multicolumn{1}{c|}{} & \multicolumn{3}{c|}{ 2D } & \multicolumn{3}{c|}{ 3D } \\
\cline { 2 - 7 } \multicolumn{1}{c|}{} & $\begin{array}{c}\text { Abstract } \\
\text { Objects }\end{array}$ & Flags & Art & $\begin{array}{c}\text { Abstract } \\
\text { Objects }\end{array}$ & Flags & Art \\
\hline Female & & & & & & \\
\hline Male & & & & & & \\
\hline
\end{tabular}

Each cell of the experimental design contains ten observations. A repeated measure design was used; all female and male subjects participated in each experimental condition. The model was also counterbalanced; each subject performed the experimental conditions in a different order to eliminate learning or fatigue effects. 


\subsection{Hypotheses}

The purpose of this thesis is to investigate if menu dimension (2D vs. 3D), content type (abstract objects, flags, art) and gender (female vs. male), are significant factors in memory retention and to determine if there are any interactions between the factors. Table 3 details the seven hypotheses that this experiment tested.

Table 3 - Hypotheses

\begin{tabular}{|c|c|}
\hline Null Hypothesis & Type \\
\hline $\begin{array}{c}\text { The population means for } \\
\text { Gender are equal }\end{array}$ & Main Effect \\
\hline $\begin{array}{c}\text { The population means for } \\
\text { Dimension are equal }\end{array}$ & Main Effect \\
\hline $\begin{array}{c}\text { The population means for } \\
\text { Content are equal }\end{array}$ & Main Effect \\
\hline $\begin{array}{c}\text { There is no interaction between } \\
\text { Gender and Dimension }\end{array}$ & First-Order Interaction \\
\hline $\begin{array}{c}\text { There is no interaction between } \\
\text { Gender and Content }\end{array}$ & First-Order Interaction \\
\hline $\begin{array}{c}\text { There is no interaction between } \\
\text { Dimension and Content }\end{array}$ & First-Order Interaction \\
\hline $\begin{array}{c}\text { There is no interaction between } \\
\text { Gender, Dimension, and Content }\end{array}$ & $\begin{array}{c}\text { Second-Order } \\
\text { Interaction }\end{array}$ \\
\hline
\end{tabular}

\subsection{Variables}

\subsubsection{Independent Variables}

There are three independent variables, dimension, content and gender. The independent variable dimension has two levels, 2D and 3D. The independent variable content has three levels; Abstract Objects, Flags, and Art. The independent variable gender has two levels, female and male. Gender was blocked since prior research has 
identified significant gender differences in various issues related to perception of websites and information processing of visual images [23].

\subsubsection{Dependent Variable}

Subjects performed an assessment test after each experimental condition that included seven questions, one for each image shown in a given carousel. The dependent variable is the number of questions the subject correctly answered on the assessment test.

The possible values for the dependent variable are $0,1,2,3,4,5,6$, or 7 .

\subsubsection{Controlled Variables}

Variables that were kept constant throughout the experiment for all subjects include:

- Location: Ergonomics Laboratory

- Lighting: window shades down to block natural light, overhead lights on

- Set-up: cubicle with a desk and two chairs, one for experimenter and one for subject

- Apparatus: laptop and wireless mouse

- Experimenter and Script

- Procedures

- Consent Form 


\subsection{Participants}

Subjects for this experiment were screened and recruited based on the following criteria:

- Background: Cal Poly Student, all majors with the exception of Software Engineering, Computer Science or Computer Engineering are welcome

- Age: 18-24 years old

- Health: Normal or corrected to normal vision (subjects who wear contacts or glasses while using a computer must wear them to the experiment)

- Experience: Little to no experience with three dimensional menus on computers

Subjects were recruited from undergraduate courses with different majors. Subjects who met the criteria were selected on a first-come, first-served basis. Twenty subjects were used in this experiment, ten females and ten males. Subject demographics including their age, major, and previous experience with $3 \mathrm{D}$ user interfaces on computers can be viewed in Appendix C. Additionally, a \$10 iTunes gift card was used as compensation for subjects' participation.

\subsection{Conditions}

The six experimental conditions (cells) are:

- 2D Abstract Objects

- 2D Flags

- $2 \mathrm{D}$ Art

- 3D Abstract Objects 
- 3D Flags

- 3D Art

Each subject first completed six training trials, one for each experimental condition, and then went through all six experimental conditions. Samples of all six experimental conditions are shown in Figure 13, 14, 15, 16, 17, and 18.
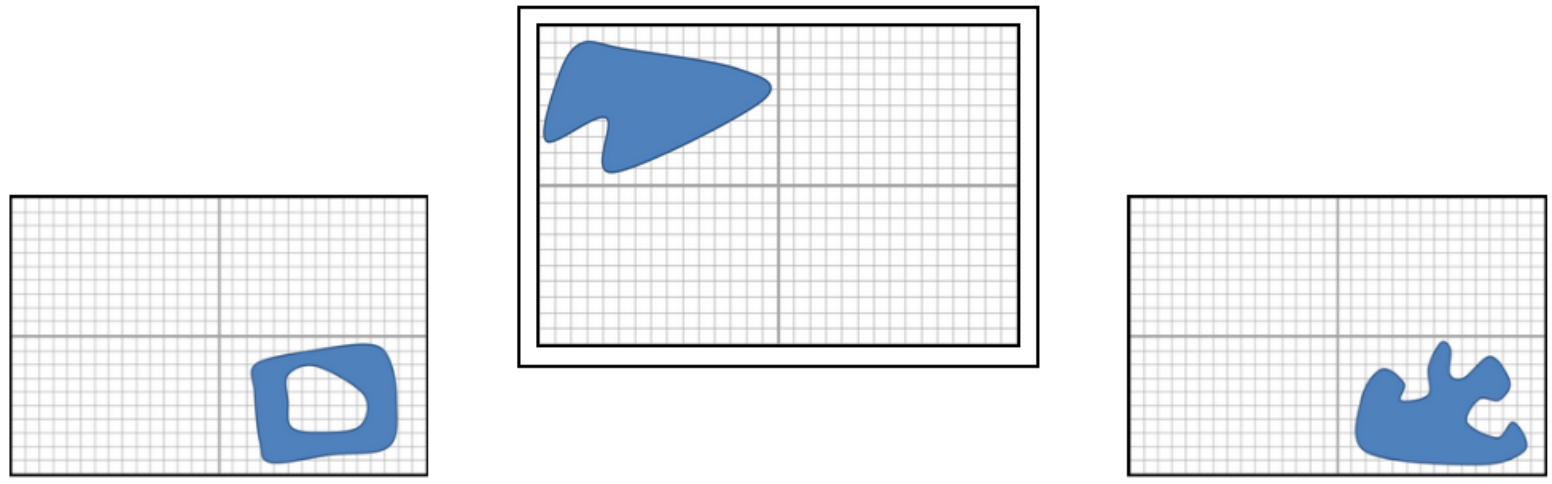

Figure 13 - Abstract Objects in 2D Carousel 


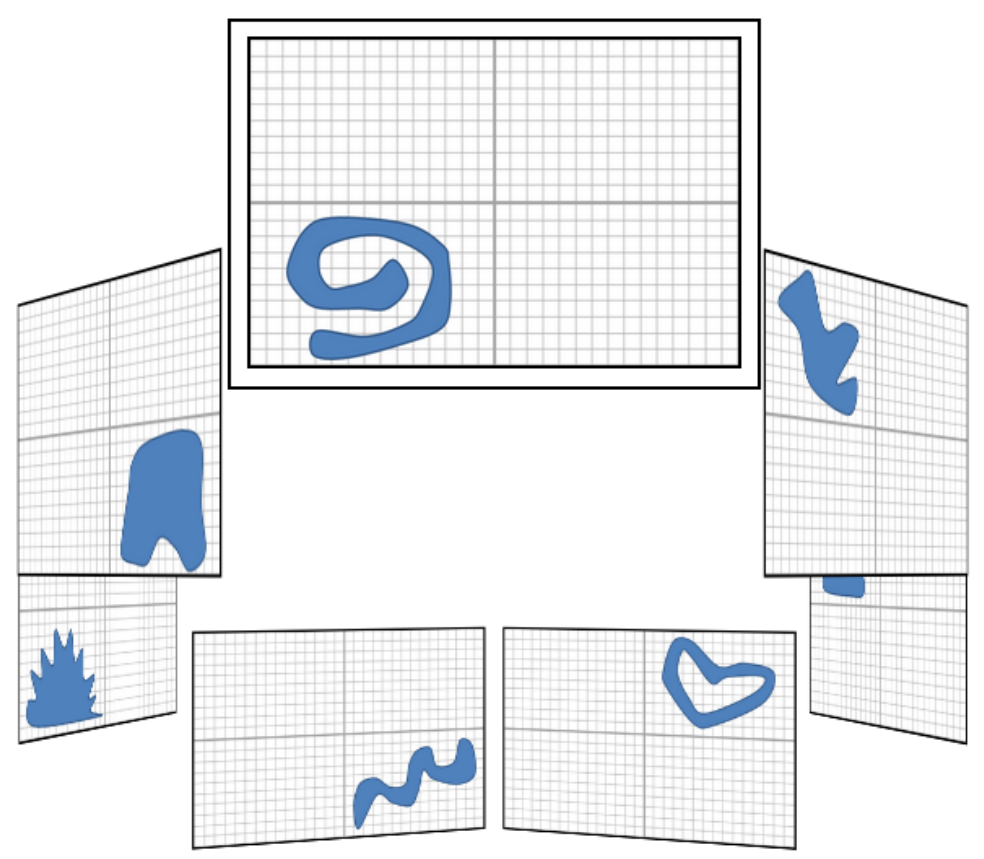

Figure 14 - Abstract Objects in 3D Carousel
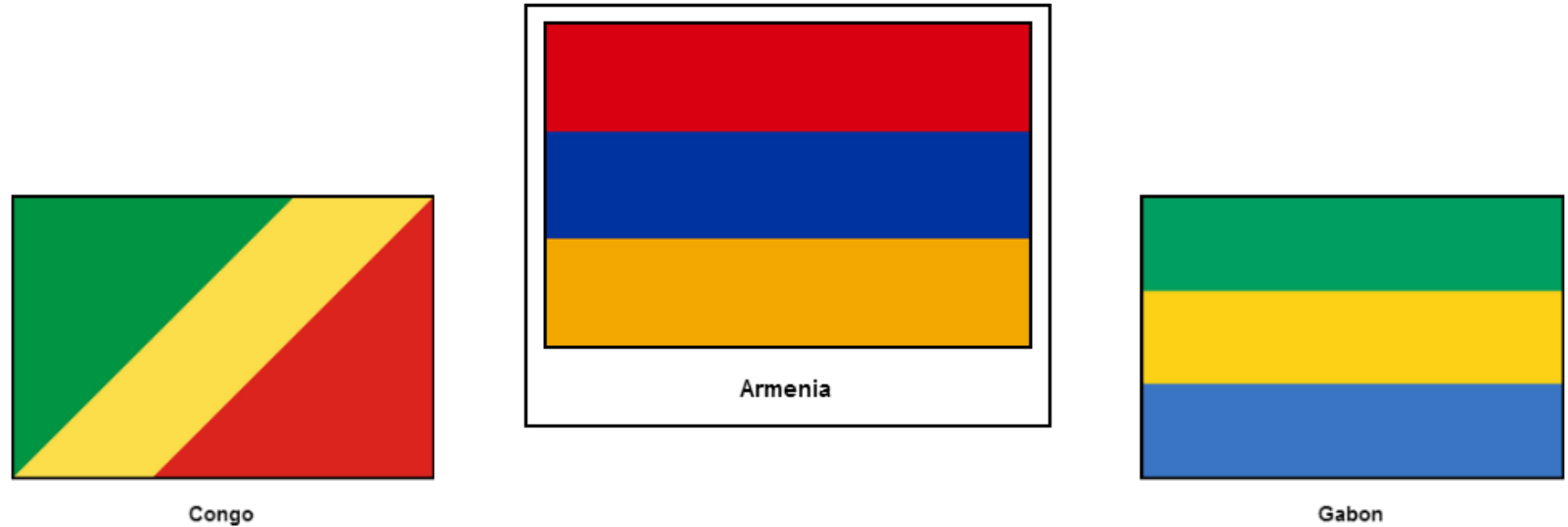

Gabon

Figure 15 - Flags in 2D Carousel

Page 28 


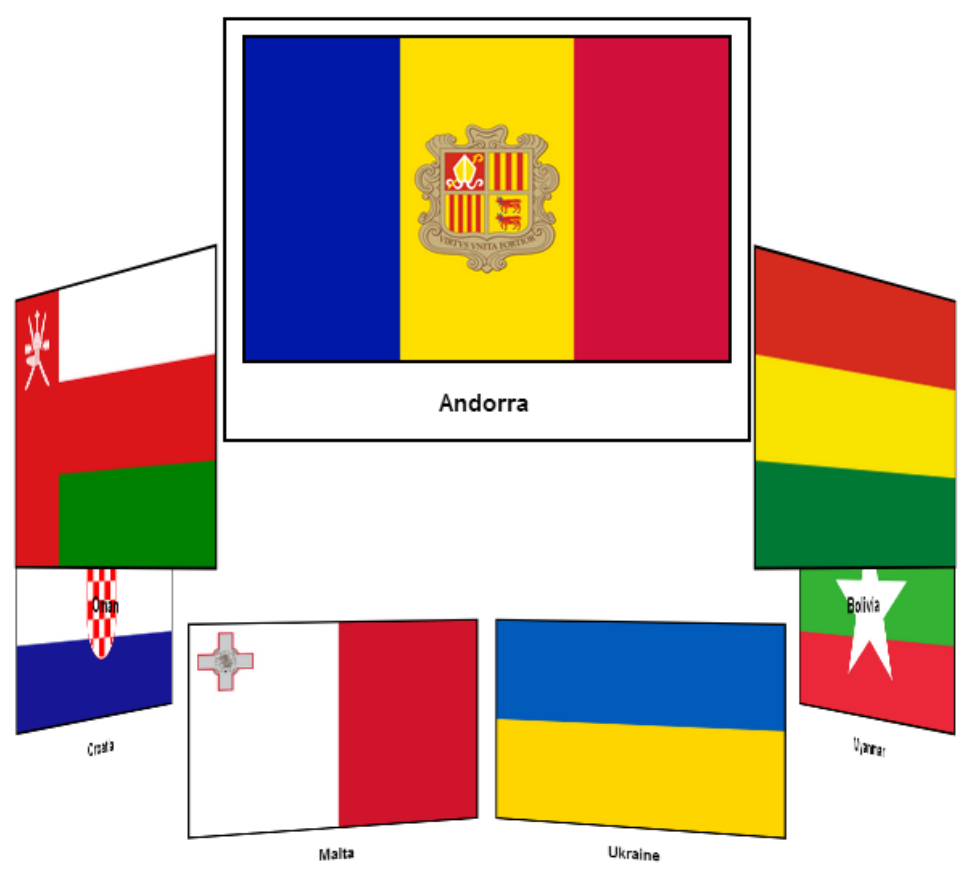

Figure 16 - Flags in 3D Carousel

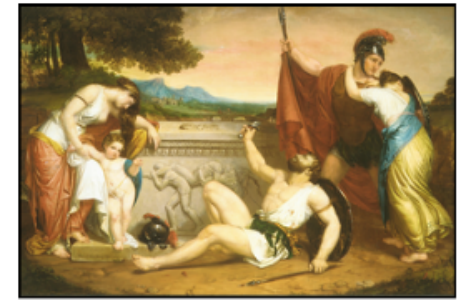

The Wages of War

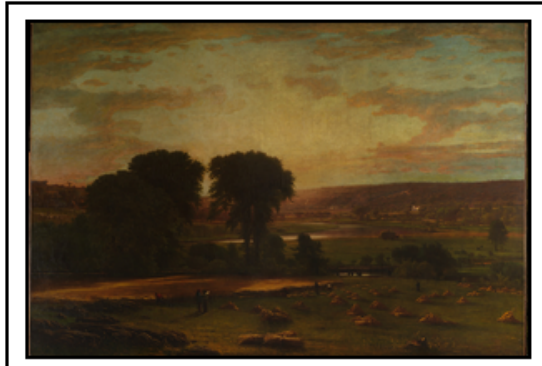

Peace and Plenty

Figure 17 - Art in 2D Carousel

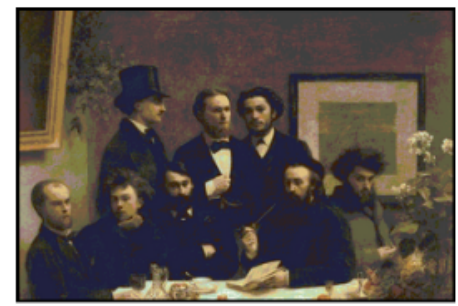

By the Table 


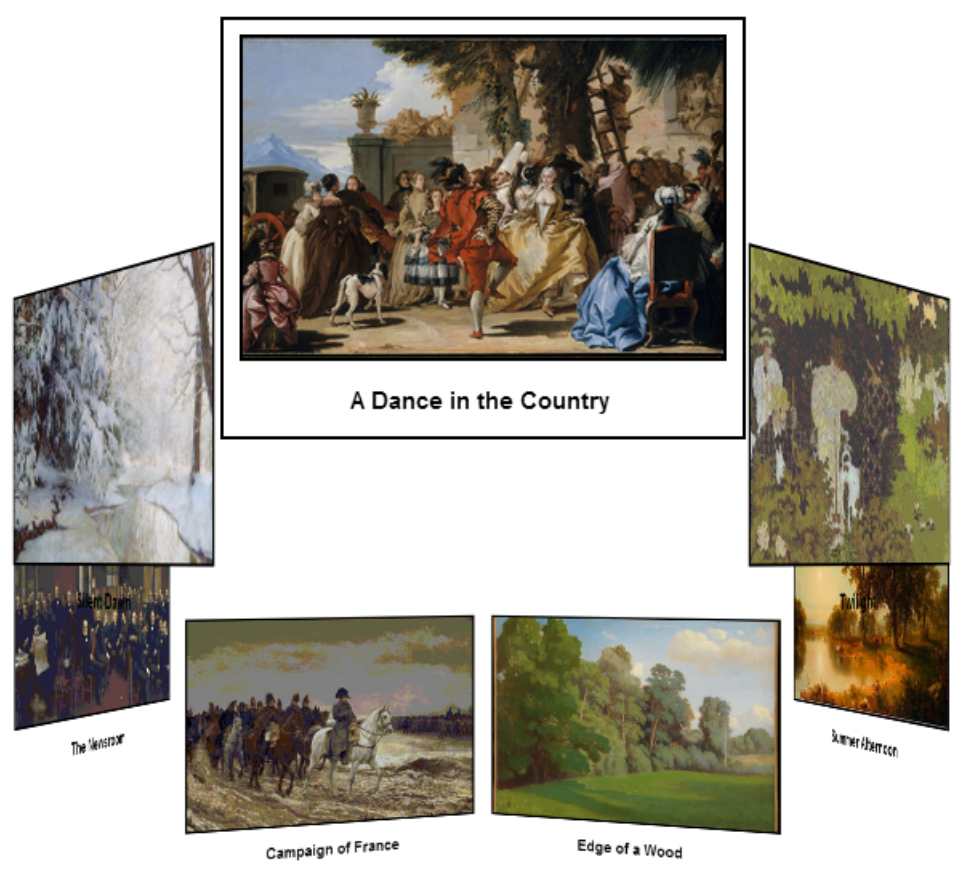

Figure 18 - Art in 3D Carousel

\subsubsection{Randomization of the Image Sets}

Between the training trials and experiment each subject was shown a total of twelve carousels, four of each content type. There was no repetition of images presented in any of the carousels so that memory retention is not positively influenced by the reappearance of images. Thus, there were four different sets of seven images for each content type. Certain image sets were not pre-determined to be presented in 2D or 3D, nor were they pre-selected to be training image sets or experimental image sets. Whether the image set was presented in $2 \mathrm{D}$ or $3 \mathrm{D}$ and in the training trials or experiment was determined by a randomization tool. The use of a randomization tool to determine the dimension an image set was presented in and whether it was training or experimental was done to reduce any potential bias that may have occurred in the difficulty level of the image sets. 


\subsection{Task}

The images in each carousel were rotating on a timer that began as soon as a subject pressed the "Start" button. Once the carousel began, subjects observed as the carousel rotated through seven images while paying attention to details of the images. Table 4 contains the nature of the question for each content type.

Table 4 - Nature of Question for Each Content Type

\begin{tabular}{|c|l|}
\hline Content Type & \multicolumn{1}{|c|}{ Nature of Question } \\
\hline Abstract Objects & Location of Object in a 4 Quadrant Grid \\
\hline Flags & Country Name, Color Scheme, Details on the Flag \\
\hline Art & Painting Name, Setting, Subject, Number of People \\
\hline
\end{tabular}

\subsection{Procedure}

\subsubsection{Pre-Experiment}

Subjects were each scheduled for thirty-minute time frames. Prior to each subject's arrival the computer was set up so that a blank subject information form was loaded on the screen. Once a subject arrived to the laboratory they were asked to place their belongings down and silence their phones. Then they were guided to the cubicle where the experiment would take place. After subjects settled into a comfortable seated position at the desk they were asked to read the instruction sheet quietly to themselves. A copy of these instructions is included in Appendix D. After reading the instructions, subjects were provided an opportunity to ask any questions they had. Next, subjects read and signed an informed consent form. A copy of the informed consent form is included in Appendix E. Once the form was signed subjects filled out a form on the computer with their basic information. Figure 19 shows a screenshot of this online form. 


\section{Please enter your information below.}

Note: All personal information will remain confidential

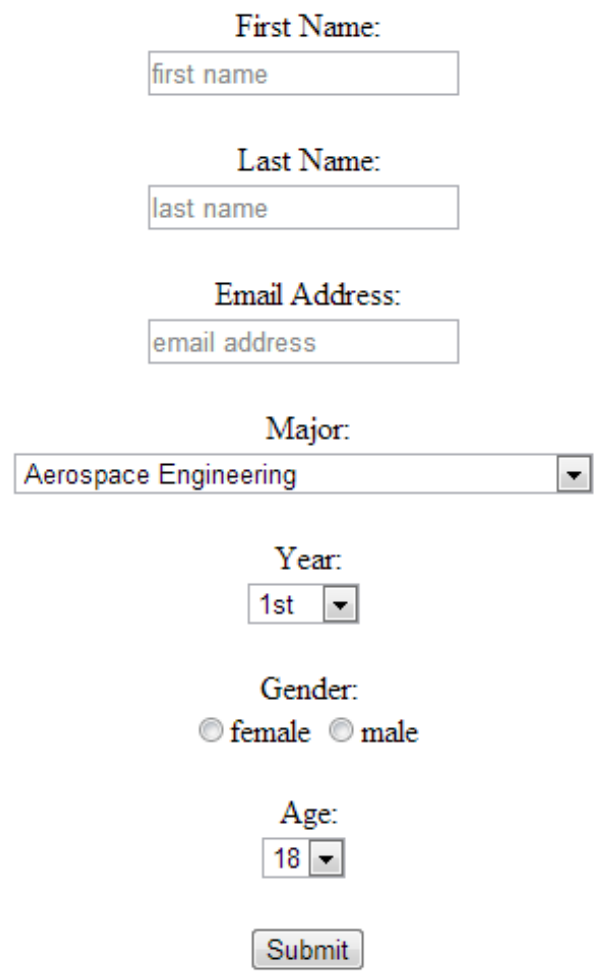

Figure 19 - Screen Shot of Subject Information Form

Once subjects completed the form and pressed the "Submit" form they proceeded to the training trials and the experiment.

\subsubsection{Training Trials and Experiment}

Subjects were presented with a main menu that included links to six training trials and six links for the experiment; a screenshot of this page is shown in Figure 20. 


\begin{tabular}{||l||}
\hline \multicolumn{1}{|c|}{ Training Trials } \\
\hline Carousel 1 \\
\hline \hline Carousel 2 \\
\hline \hline Carousel 3 \\
\hline \hline Carousel 4 \\
\hline \hline Carousel 5 \\
\hline Carousel 6 \\
\hline
\end{tabular}

\begin{tabular}{||c||}
\hline \multicolumn{1}{|c|}{ Experiment } \\
\hline Carousel 7 \\
\hline \hline Carousel 8 \\
\hline \hline Carousel 9 \\
\hline \hline Carousel 10 \\
\hline \hline Carousel 11 \\
\hline Carousel 12 \\
\hline
\end{tabular}

Survey

Figure 20 - Screen Shot of Tables

Once subjects reached this page they were told that they would first perform the six training trials in the order listed. When a link was clicked it would open a new tab, subjects would then press a "Start" button on that page and watch the carousel present seven images, then answer the seven questions pertaining to that carousel. There was no time limit on the questions. When they were finished with a carousel's assessment they would close the tab and return to the page with the table and repeat that process for all six training trials. After the training trials subjects were given an opportunity to ask any questions they had before continuing to the experiment. Once questions were answered, subjects were instructed to proceed to the experiment in the same manner that they performed the training trials. 


\subsubsection{Post-Experiment}

Once the experiment was complete, subjects were instructed to click the "Survey" button at the bottom of their screen. The qualitative survey contained a question about each of the six carousels they were shown in the experiment. Subjects were provided with the carousel that was being referred to in the question and were asked to "Rank the effectiveness of this display orientation for your retention of the content." These questions were asked one at a time and in the order that the carousels were presented in the experiment. Figure 21 shows a sample of the question with a 2D Abstract Objects carousel.
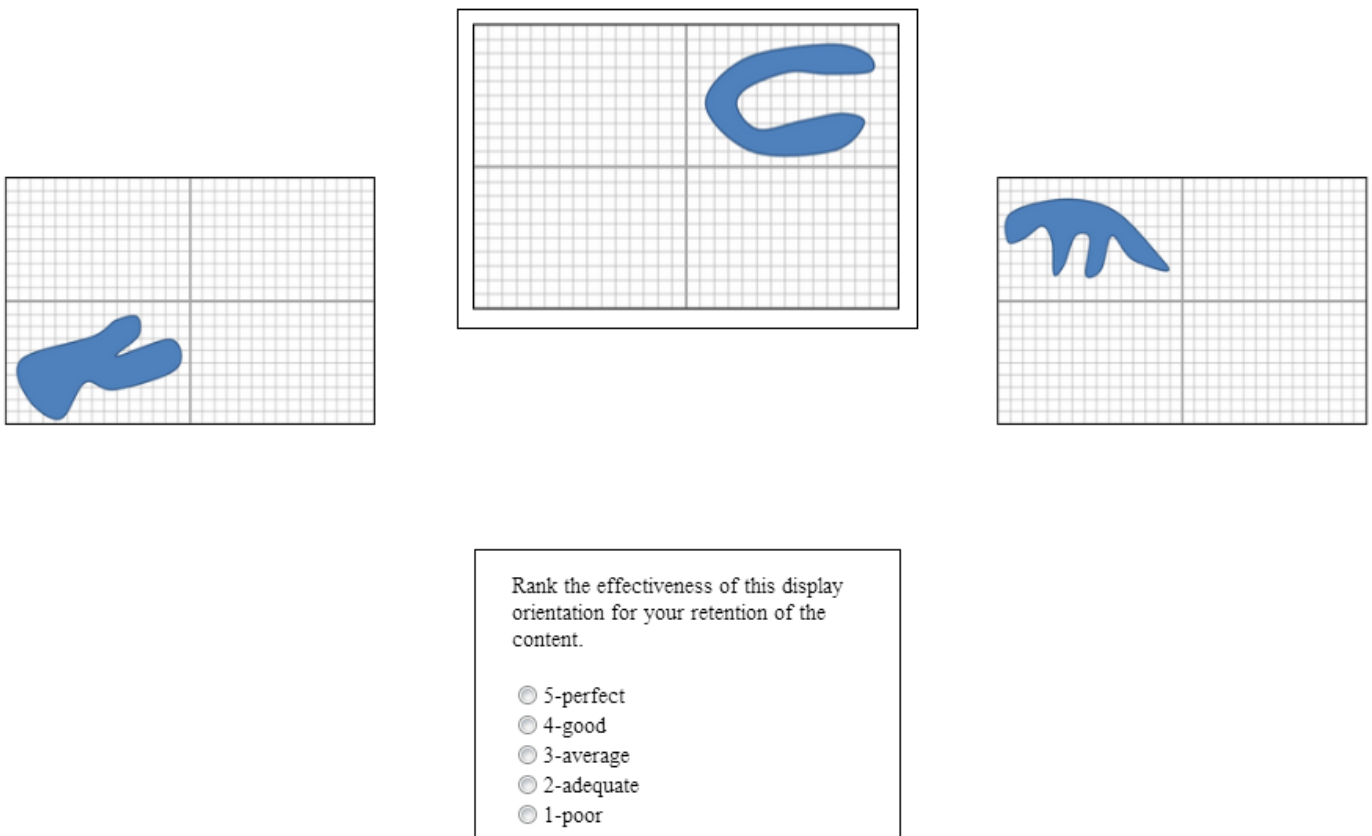

Next

Figure 21 - Sample of Carousel Ranking Question 
After ranking all six of the carousels, each subject was asked to comment on the effectiveness of 2D and 3D displays on their memory retention. Figure 22 shows a screenshot of this question.

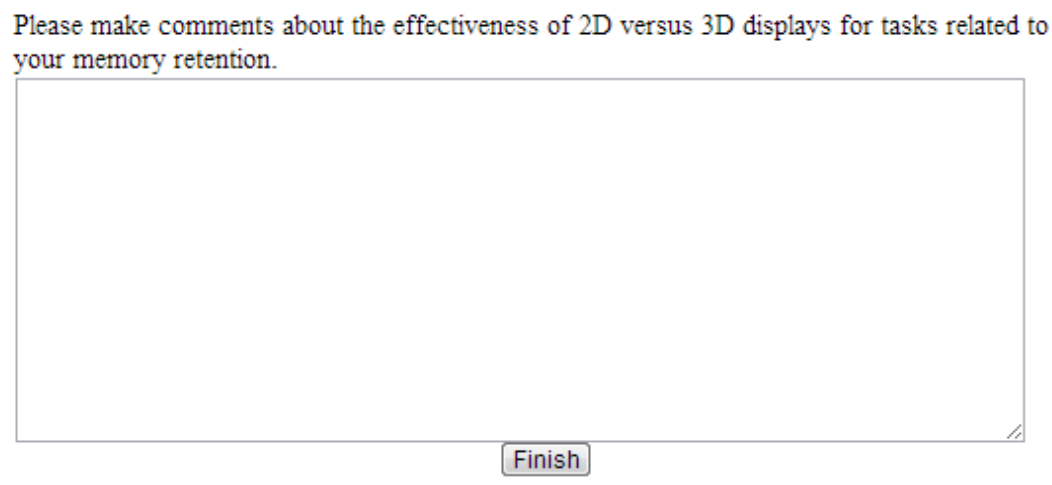

Figure 22 - Final Questionnaire Question

When subjects were done writing their comments they would press the "Finish" button. At this point the experiment and questionnaire were complete; subjects were then thanked for their participation and given their \$10 iTunes gift card.

\subsection{Experimental Setting and Apparatus}

The experiment was conducted in a cubicle of the Ergonomics Laboratory in the Industrial and Manufacturing Engineering Department. The cubicle contained a desk and two chairs. The chair at the desk was for the participant and the second chair was placed slightly off to the side behind the subject for the experimenter to observe. On the desk was the laptop used throughout the experiment as well as a wireless mouse. The laptop used had a 15.6" high definition, LED LCD. To prevent distraction, no other items were allowed in the cubicle during the experiment. To accommodate subjects' varying heights and comfort each subject was allowed to adjust the chair height and the angle of the laptop display before the experiment began. 


\subsection{Pilot}

Prior to running the experiment a pilot was run to determine the boundaries of the experiment and confirm the assumptions. There were six participants in the pilot, three male and three female. The pilot confirmed the assumptions about the experimental setup, particularly regarding each content type:

- Abstract Objects: This content type was manageable for all subjects; some subjects were able to achieve a seven out of seven score on the assessment. Although this content type was the easiest of the three selected, not all subjects achieved perfect scores on the assessment.

- Flags: This content type was the most difficult for participants in the pilot, although many subjects commented on the difficulty due to the lack of discriminability between country flags and the amount of details to remember, their scores showed that they were still able to correctly answer a number of the questions.

- Art: This content type was suitable for the subjects. Although it was more difficult than the abstract objects, scores were generally higher than the flag scores. 


\section{CHAPTER 5: RESULTS}

\subsection{Quantitative Results}

This section details the analysis of the quantitative results from the experiment. The response variable for the quantitative results is the number of questions the subject answered correctly out of a possible seven. First descriptive statistics were run on the data, and a test of normality was performed to determine if the results were normally distributed since ANOVA tests assume that the residuals are normally distributed. Finally a repeated measure ANOVA test was performed on the data and interaction plots were generated.

\subsubsection{Raw Data and Descriptive Statistics}

The raw quantitative data can be seen in Appendix F. Table 5 shows the descriptive statistics for the raw quantitative data. The grand mean for the data set is 5.075 correct responses; this value was used to calculate the residuals.

Table 5 - Descriptive Statistics for Quantitative Response Variable

\begin{tabular}{|l|c|}
\hline \multicolumn{2}{|c|}{ Descriptive Statistics: Response } \\
\hline $\mathrm{N}$ & 120 \\
\hline Mean* & 5.075 \\
\hline Standard Deviation* & 1.562 \\
\hline Minimum* & 1.000 \\
\hline Q1* & 4.000 \\
\hline Median* & 5.000 \\
\hline Q3* & 6.000 \\
\hline Maximum* & 7.000 \\
\hline Skewness & -0.52 \\
\hline Kurtosis & -0.58 \\
\hline
\end{tabular}

*Units: number of correct responses 
A histogram of the response data is shown in Figure 23. A skewness value of -.52 denotes a negative skew in the data. The histogram also shows that the data has a slight negative skewness with the most frequent responses occurring to the right of the figure. A Kurtosis value of -0.58 indicates that the responses are flatter than normal.

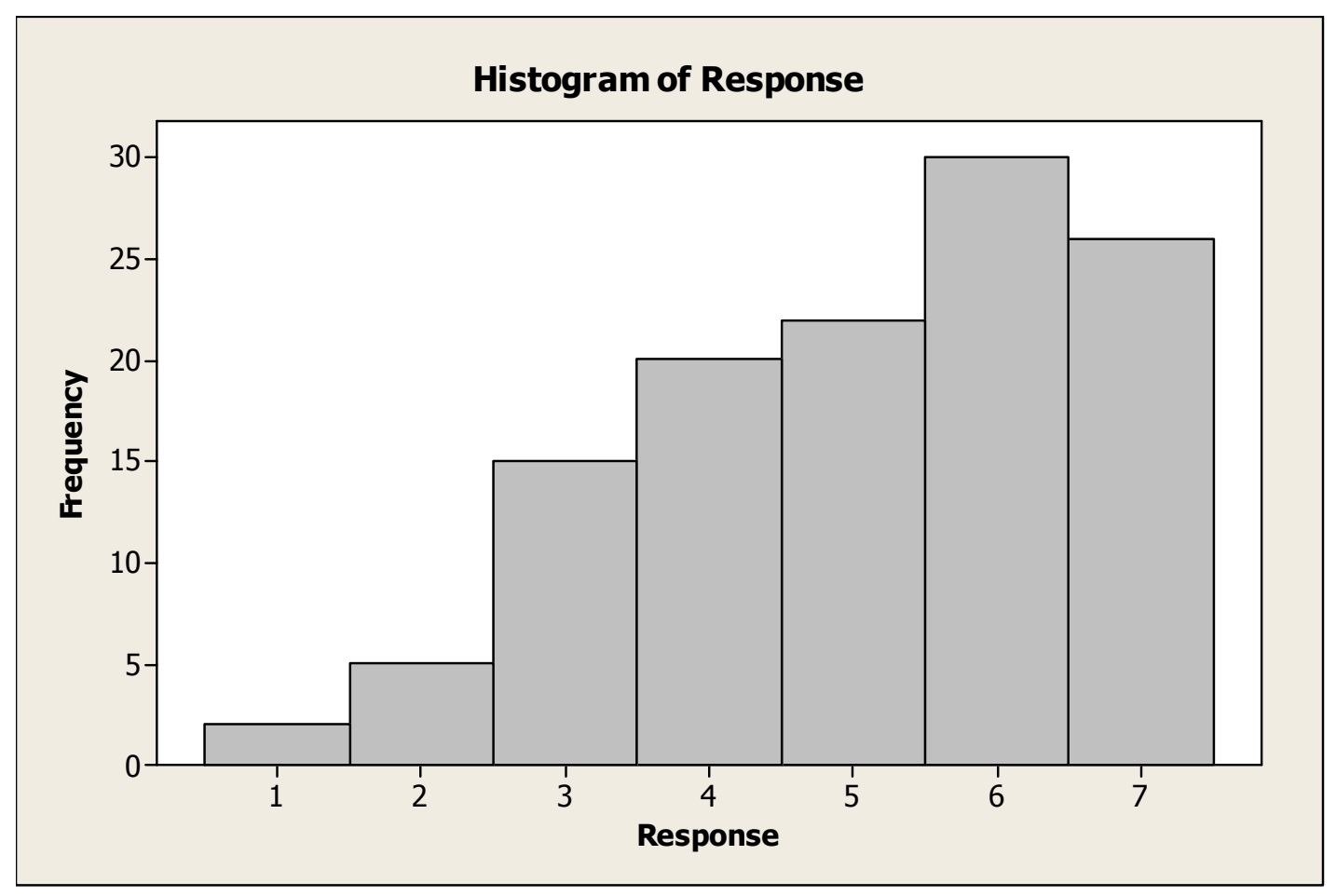

Figure 23 - Histogram of Quantitative Responses

\subsubsection{Test of Normality}

The Ryan-Joiner test of normality was chosen due to its high power of testing. The Ryan-Joiner test of normality for the residuals of the response variable generated a Pvalue of $>0.100$ showing that the residuals are distributed normally. Using this test the ANOVA assumption of normality is satisfied. 


\subsubsection{ANOVA Test}

This section summarizes the ANOVA test performed on the quantitative data. The ANOVA model is a three factor split-plot (one-between subject factor and two-within subject factors) model. Details of the levels for each factor are shown in Table 6.

Table 6 - ANOVA Factors and Levels

\begin{tabular}{|c|c|}
\hline Factor & Gender \\
\hline Level 1 & Female \\
\hline Level 2 & Male \\
\hline
\end{tabular}

\begin{tabular}{|c|c|}
\hline Factor & Dimension \\
\hline Level 1 & 2D \\
\hline Level 2 & 3D \\
\hline
\end{tabular}

\begin{tabular}{|c|c|}
\hline Factor & Content \\
\hline Level 1 & $\begin{array}{c}\text { Abstract } \\
\text { Objects }\end{array}$ \\
\hline Level 2 & Flags \\
\hline Level 3 & Art \\
\hline
\end{tabular}

Table 7 displays the test results for the sources of random effect.

Table 7 - Random Effect Variance Component Estimates

\begin{tabular}{|c|c|c|c|c|c|c|}
\hline Random Effect & $\begin{array}{c}\text { Variance } \\
\text { Ratio }\end{array}$ & $\begin{array}{c}\text { Variance } \\
\text { Component }\end{array}$ & $\begin{array}{c}\text { Std } \\
\text { Error }\end{array}$ & $\begin{array}{c}\mathbf{9 5 \%} \\
\text { Lower }\end{array}$ & $\begin{array}{c}\text { 95\% } \\
\text { Upper }\end{array}$ & $\begin{array}{c}\text { Percent } \\
\text { of } \\
\text { Total }\end{array}$ \\
\hline Subject & 0.071 & 0.130 & 0.151 & -0.167 & 0.426 & 4.581 \\
\hline Content & 0.410 & 0.745 & 0.831 & -0.883 & 2.337 & 26.338 \\
\hline Gender*Dimension & 0.074 & 0.135 & 0.227 & -0.309 & 0.580 & 4.781 \\
\hline Content*Dimension & -0.039 & -0.071 & 0.025 & -0.119 & -0.023 & 0.000 \\
\hline Residual & & 1.819 & 0.271 & 1.386 & 2.494 & 64.300 \\
\hline Total & & 2.830 & 0.871 & 1.676 & 5.768 & 100.000 \\
\hline
\end{tabular}

From the variance component analysis, it can be noted that the largest source of variability aside from the residual is content. Content contributes to 26.338 percent of the total variability, however since zero is contained in the $95 \%$ confidence interval for the variance component, content is not statistically significant. Table 8 displays the fixed effect test results. 
Table 8 - Fixed Effect Test Results

\begin{tabular}{|l|c|c|c|}
\hline \multicolumn{1}{|c|}{ Source } & DF & F Ratio & Prob > F \\
\hline Gender & 1 & 0.0141 & 0.9138 \\
\hline Dimension & 1 & 0.0204 & 0.8995 \\
\hline Gender*Dimension & 1 & 0.0046 & 0.9462 \\
\hline Gender*Content*Dimension & 2 & 1.1038 & 0.3360 \\
\hline
\end{tabular}

Gender and Dimension are not significant main effects, with large P-values of 0.9138 and 0.8995 respectively. There is no significant interaction between gender and dimension (P-value of 0.9462), and the second-order interaction of Gender*Content*Dimension is also not significant with a P-value of 0.3360 .

\subsubsection{Interaction Plots}

This section includes the interaction plots for the first order interactions and the second order interaction. There are three first order interactions: Gender vs. Dimension, Gender vs. Content, and Content vs. Dimension. The interaction plots for first order interactions are shown below in Figure 24, 25, and 26.

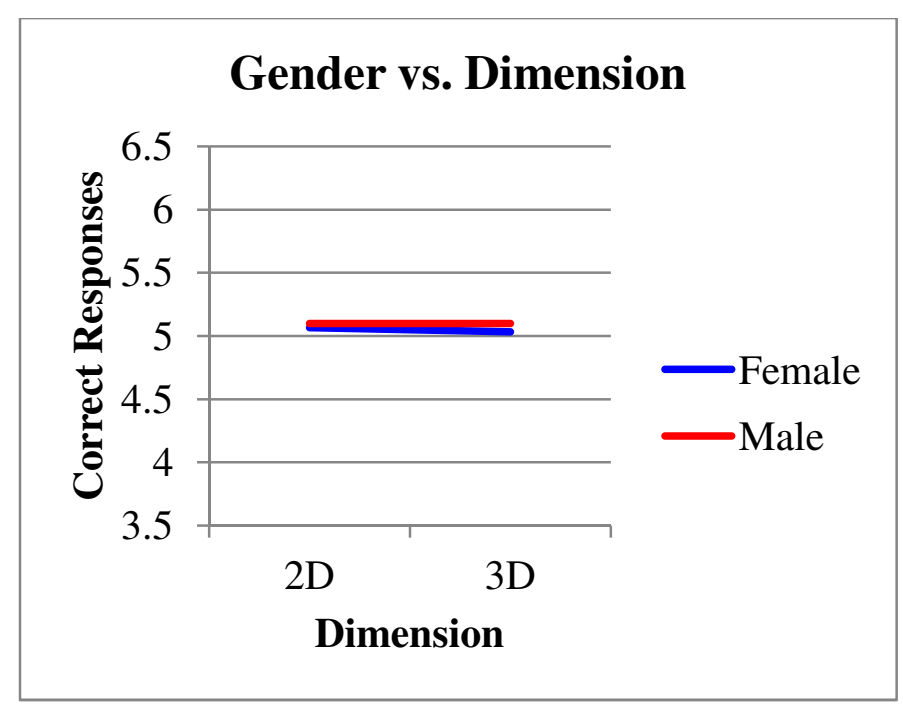

Figure 24 - Gender vs. Dimension Interaction Plot 
The interaction plot of Gender vs. Dimension (Figure 24) shows parallelism of the lines, demonstrating that there is no interaction. The means are about the same for females and males across both dimensions showing that gender and dimension are not significant main effects.

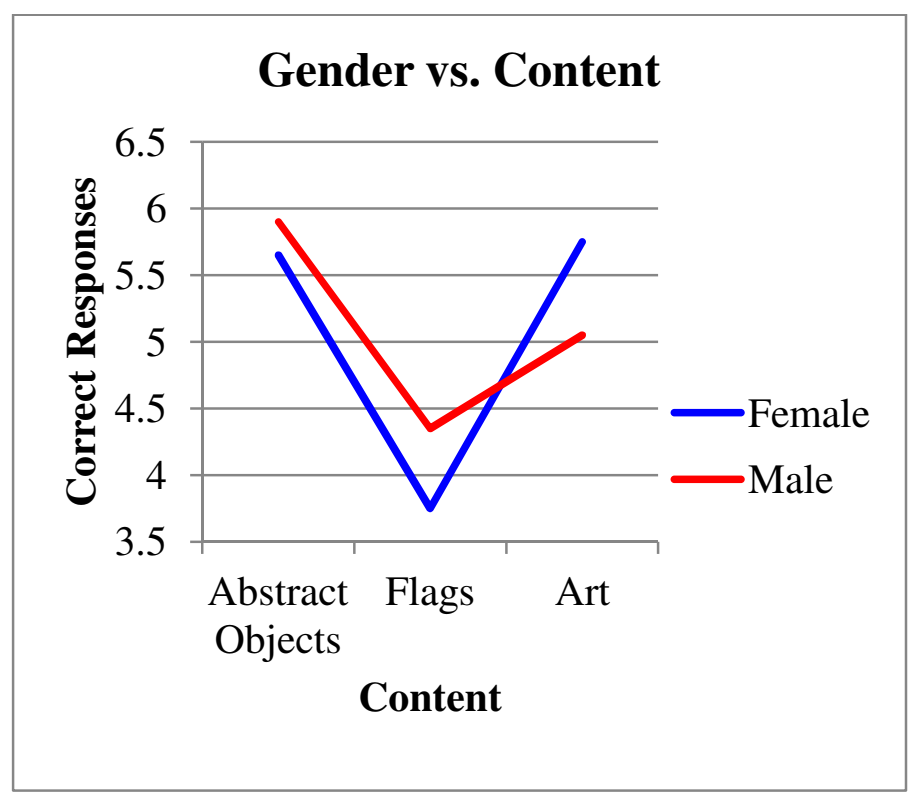

Figure 25 - Gender vs. Content Interaction Plot

The interaction plot of Gender vs. Content shows nearly parallel lines, demonstrating that there is no interaction. The means differ across content types in approximately the same pattern for both females and males, demonstrating that content is a significant main effect as shown above. The means were relatively high for abstract objects, then decreased for flags, and increased again for art. 


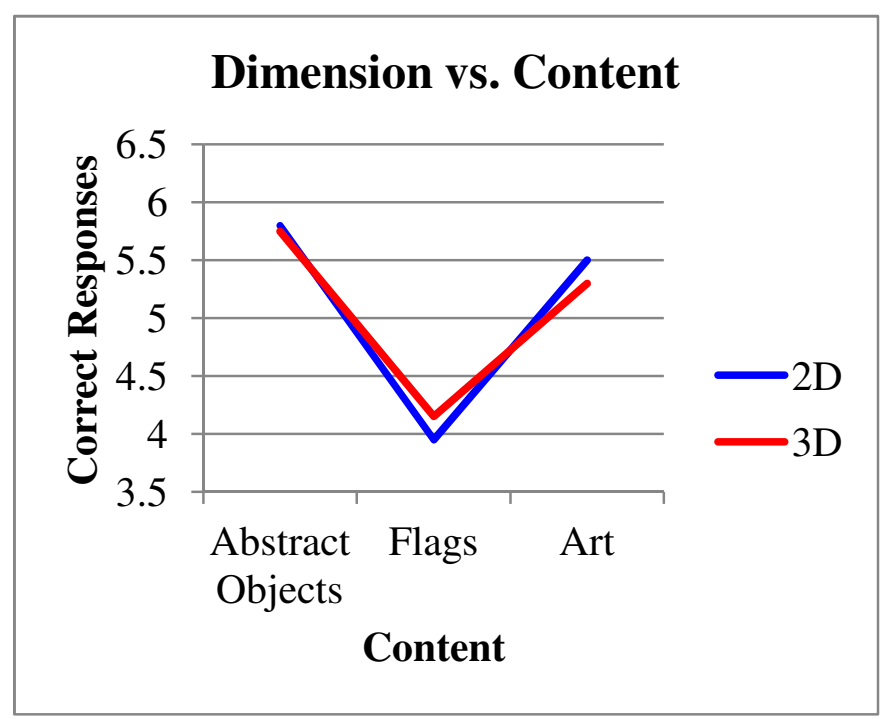

Figure 26 - Dimension vs. Content Interaction Plot

The interaction plot of Dimension vs. Content shows parallelism of the lines,

demonstrating that there is no interaction. The means for each content type are about the same across both dimensions showing dimension is not significant main effects. There is one second order interaction term: Gender vs. Dimension vs. Content. The three way interaction plot can be seen in Figure 27. However, this interaction was not significant (Pvalue of 0.3360$)$.

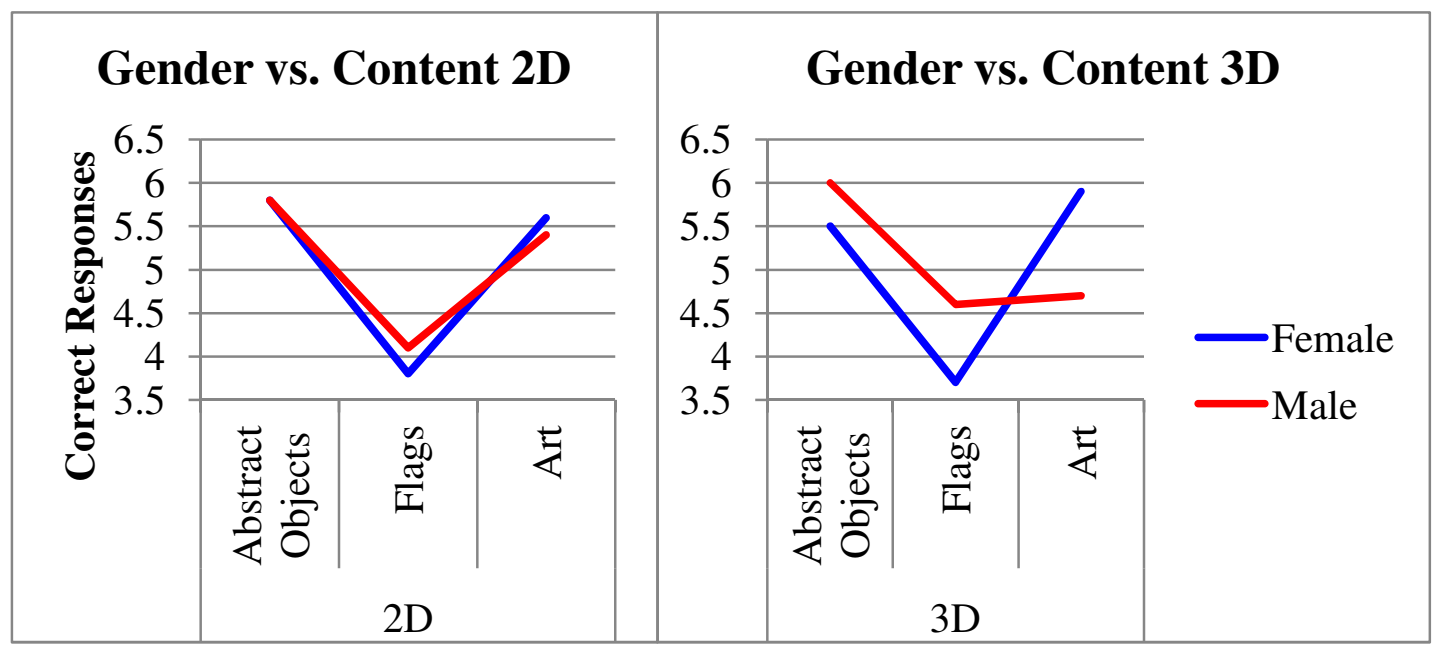

Figure 27 - Gender vs. Dimension vs. Content 
In the interaction plot on the left for $2 \mathrm{D}$, there is parallelism in the lines and roughly the same mean values for males and females across all three content types. However, looking at the interaction plot on the right for $3 \mathrm{D}$ there is a slightly different pattern. With $2 \mathrm{D}$ the means were relatively high for abstract objects, then decreased for flags, and increased again for art, and followed the same pattern in 3D for females. For males however, in the 3D plot, the mean for art increased much less than it did for females. The mean for females in 3D increased from flags to art by 2.2 correct responses, while for the same stimuli the male mean only increased by 0.1 correct responses. This interaction is not statistically significant.

\subsection{Qualitative Results}

This section details the analysis of the questionnaire results from the experiment. At the end of the experiment each subject was asked to rank the effectiveness of each of the six carousels. The question posed for each carousel was: "Rank the effectiveness of this display orientation for your retention of the content." The response variable for the questionnaire results was a ranking with a scale of 1-5 (1-poor, 2-adequate, 3-average, 4good, 5-perfect). Although this response variable is a categorical variable, it is treated as continuous for this analysis which is typical for likert scales. First descriptive statistics were run on the data, and the residuals were tested for normality. An ANOVA test was performed on the data and interaction plots were generated.

\subsubsection{Raw Data and Descriptive Statistics}

The raw data from the questionnaire can be seen in Appendix G. Table 9 shows the descriptive statistics for the raw questionnaire data. The grand mean for the data set is a rating of 3.4667; this value was used to calculate the residuals for the normality test. 
Table 9 - Descriptive Statistics for Questionnaire Response Data

\begin{tabular}{|l|c|}
\hline \multicolumn{2}{|c|}{$\begin{array}{c}\text { Descriptive Statistics: } \\
\text { Response }\end{array}$} \\
\hline $\mathrm{N}$ & 120 \\
\hline Mean* & 3.4667 \\
\hline Standard Deviation* & 1.0919 \\
\hline Minimum* & 1.0000 \\
\hline Q1* & 3.0000 \\
\hline Median* & 4.0000 \\
\hline Q3* & 4.0000 \\
\hline Maximum* & 5.0000 \\
\hline Skewness & -0.62 \\
\hline Kurtosis & -0.18 \\
\hline *Units* Rating &
\end{tabular}

*Units: Rating

A histogram of the response data is shown in Figure 28 - Histogram of

Questionnaire Responses. A skewness value of -0.62 denotes a negative skew in the data.

The histogram also shows that the data has a slight negative skewness with the most

frequent responses occurring in the right of the figure. A Kurtosis value of -0.18 indicates that the responses are slightly flatter than normal. 


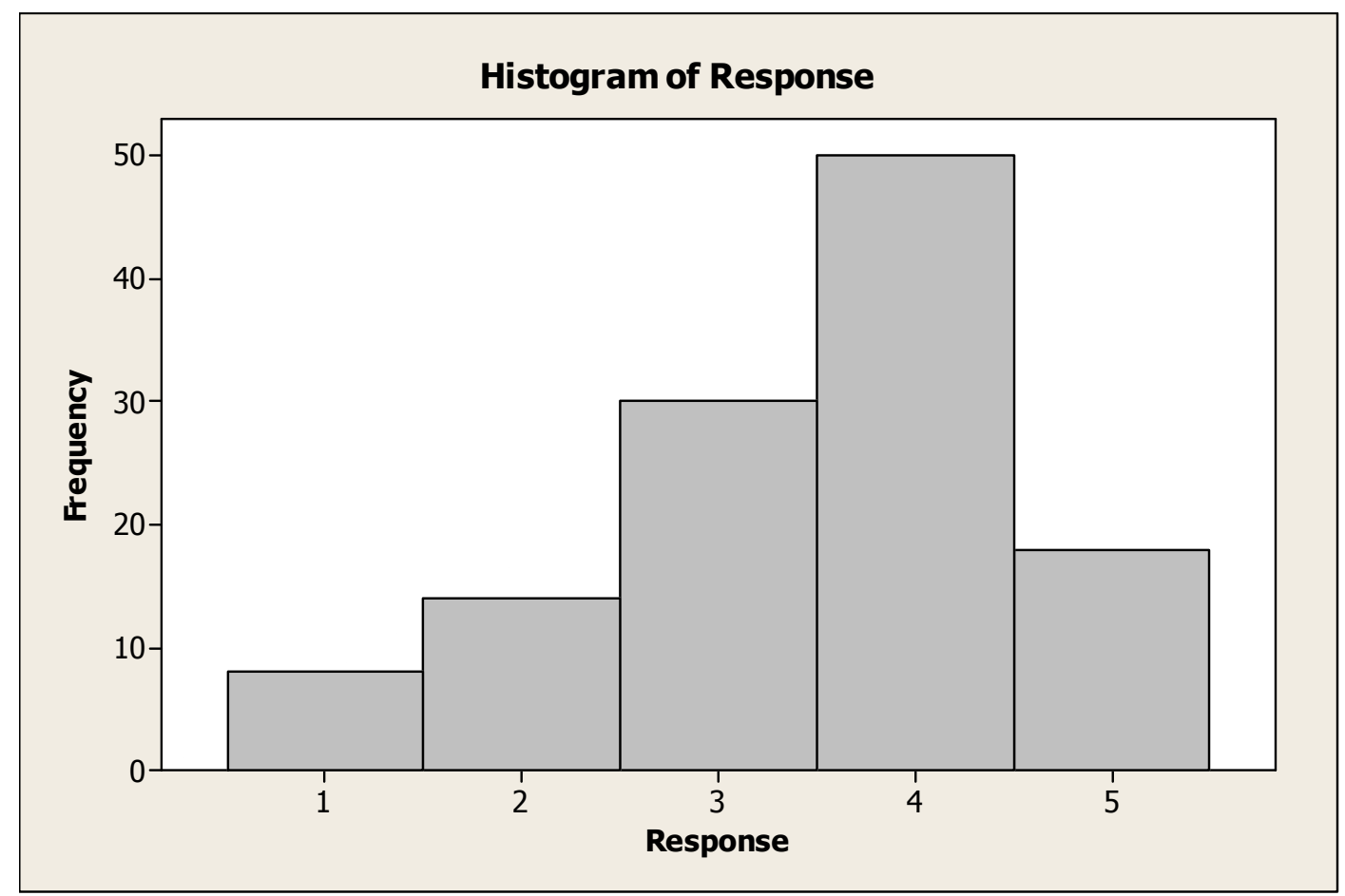

Figure 28 - Histogram of Questionnaire Responses

\subsubsection{Test of Normality}

The Ryan-Joiner test of normality was chosen due to its high power of testing. The Ryan-Joiner test of normality for the residuals of the response variable generated a Pvalue of $>0.100$ showing that the residuals are distributed normally. Using this test the ANOVA assumption of normality is satisfied.

\subsubsection{ANOVA Test}

This section summarizes the ANOVA test performed on the questionnaire data. The ANOVA model is a three factor split-plot (one-between subject factor and twowithin subject factors) model. Details of the levels for each factor are shown in Table 10. 
Table 10 - ANOVA Factors and Levels

\begin{tabular}{|c|c|}
\hline Factor & Gender \\
\hline Level 1 & Female \\
\hline Level 2 & Male \\
\hline
\end{tabular}

\begin{tabular}{|c|c|}
\hline Factor & Dimension \\
\hline Level 1 & 2D \\
\hline Level 2 & 3D \\
\hline
\end{tabular}

\begin{tabular}{|c|c|}
\hline Factor & Content \\
\hline Level 1 & $\begin{array}{c}\text { Abstract } \\
\text { Objects }\end{array}$ \\
\hline Level 2 & Flags \\
\hline Level 3 & Art \\
\hline
\end{tabular}

Table 11 displays the test results for the sources of random error.

Table 11 - Random Effect Variance Component Estimates

\begin{tabular}{|c|c|c|c|c|c|c|}
\hline Random Effect & $\begin{array}{c}\text { Variance } \\
\text { Ratio }\end{array}$ & $\begin{array}{c}\text { Variance } \\
\text { Component }\end{array}$ & $\begin{array}{c}\text { Std } \\
\text { Error }\end{array}$ & $\begin{array}{c}\text { 95\% } \\
\text { Lower }\end{array}$ & $\begin{array}{c}\text { 95\% } \\
\text { Upper }\end{array}$ & $\begin{array}{c}\text { Percent of } \\
\text { Total }\end{array}$ \\
\hline Subject & 0.145 & 0.143 & 0.105 & -0.063 & 0.350 & 10.319 \\
\hline Content & -0.135 & -0.133 & 0.155 & -0.437 & 0.170 & 0.000 \\
\hline Gender*Dimension & -0.029 & -0.029 & 0.022 & -0.071 & 0.014 & 0.000 \\
\hline Content*Dimension & 0.263 & 0.259 & 0.309 & -0.346 & 0.865 & 18.677 \\
\hline Residual & & 0.986 & 0.147 & 0.751 & 1.352 & 71.004 \\
\hline Total & & 1.389 & 0.345 & 0.900 & 2.421 & 100.000 \\
\hline
\end{tabular}

From the variance component analysis, it can be noted that the largest source of variability aside from the residual is the interaction of Content*Dimension. Content*Dimension contributes to 18.677 percent of the total variability, however since zero is contained in the $95 \%$ confidence interval for the variance component, Content*Dimension is not statistically significant. Content and the interaction of Gender*Content both contribute 0.000 percent to the total variability, indicating that they are not significant. Table 12 displays the fixed effect test results. 
Table 12 - Fixed Effect Test Results

\begin{tabular}{|l|c|c|c|}
\hline \multicolumn{1}{|c|}{ Source } & DF & F Ratio & Prob > F \\
\hline Gender & 1 & 4.4415 & 0.0823 \\
\hline Dimension & 1 & 0.0486 & 0.8460 \\
\hline Gender*Dimension & 1 & 0.1352 & 0.7140 \\
\hline Gender*Content*Dimension & 2 & 0.0591 & 0.9426 \\
\hline
\end{tabular}

Gender is a notable main effect with a P-value of 0.0823 , but it is not significant based on an alpha level of 0.05. Dimension is also not a significant main effect, with large P-values of 0.8460 . There is no significant interaction between gender and dimension (P-value 0.7140), and the second-order interaction of Gender*Content*Dimension is also not significant with a P-value of 0.9426 .

\subsubsection{Interaction Plots}

This section includes the means and all of the interaction plots for the first order interactions and the second order interaction. There are three first-order interactions:

Gender vs. Dimension, Gender vs. Content, and Content vs. Dimension. The interaction plots for first-order interactions are listed below in Figure 29, 30, and 31. 


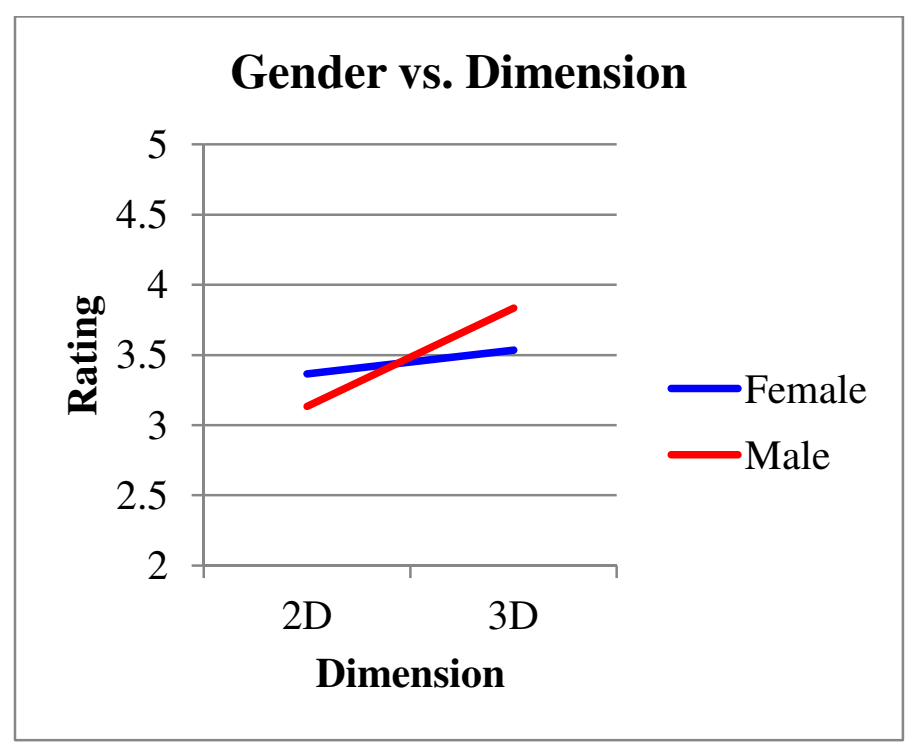

Figure 29 - Gender vs. Dimension Interaction Plot

The interaction plot of Gender vs. Dimension (Figure 29) shows a lack of parallelism suggesting that there may be some interaction between gender and dimension. The Pvalue of 0.7140 , however, indicates that this is not a significant interaction.

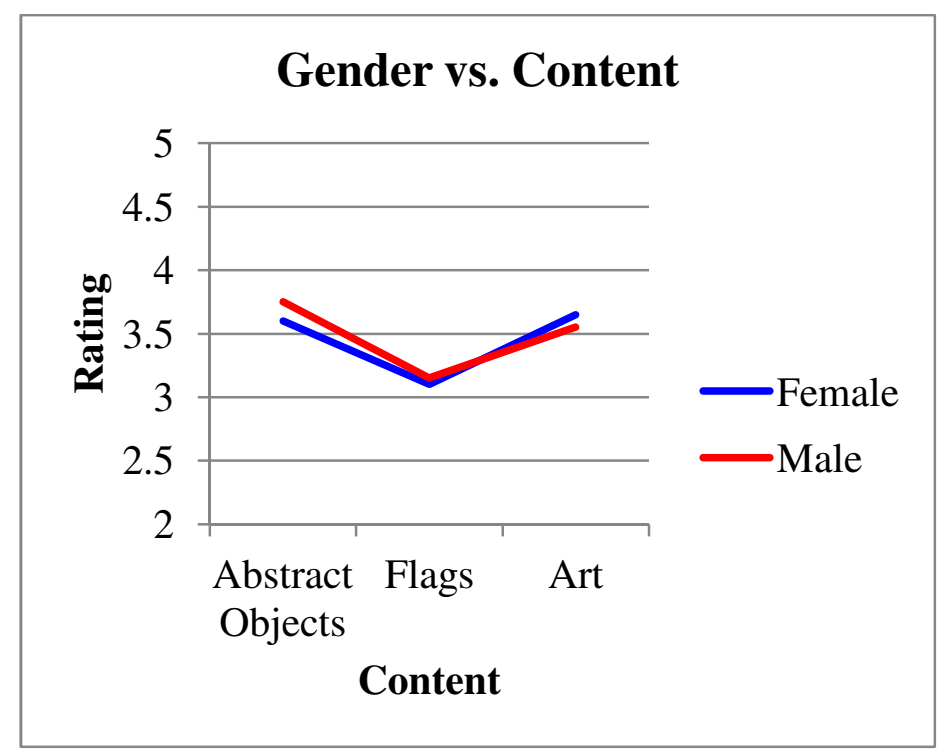

Figure 30 - Gender vs. Content Interaction Plot 
The interaction plot of Gender vs. Content (Figure 30) shows parallelism of the lines, demonstrating that there is no interaction. The means for the Abstract Objects, Flags, and Art are 3.675, 3.125, and 3.6 respectively. There is little variation in the means across content types indicating that content is not a significant main effect.

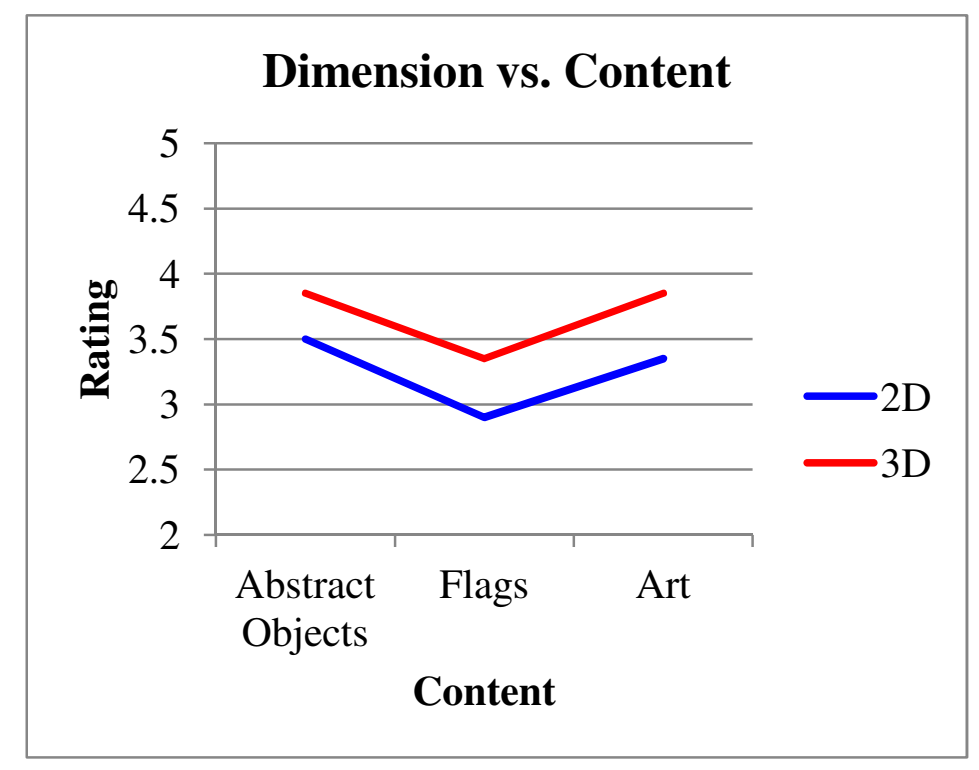

Figure 31 - Dimension vs. Content Interaction Plot

The interaction plot of Dimension vs. Content (Figure 31) shows parallelism of the lines. The interaction of Dimension*Content contributed to 18.677 percent of the total variability. There is one second order interaction term: Gender vs. Dimension vs. Content. The three way interaction plot can be seen in Figure 32.However this interaction was not significant (P-value of 0.9426). 


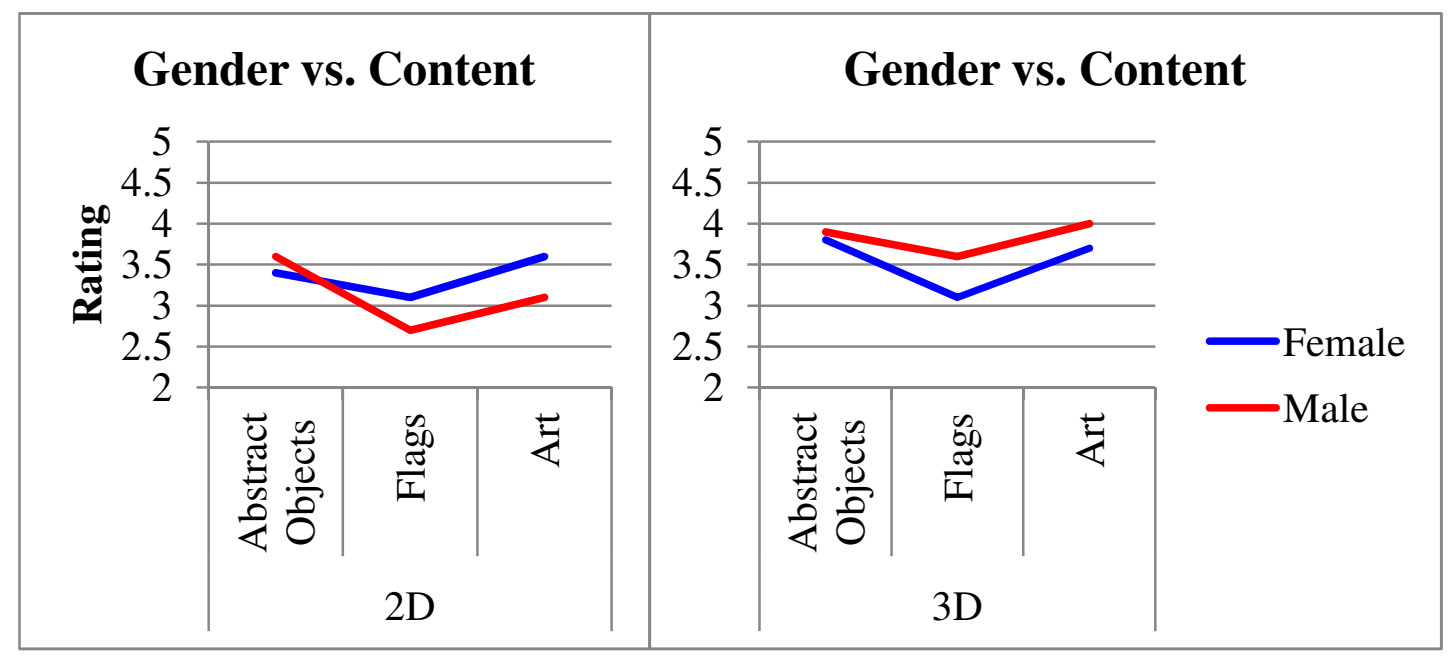

Figure 32 - Gender vs. Content vs. Dimension Interaction Plot

In the interaction plot on the right for 3D there is parallelism in the lines and approximately the same mean values for males and females across all three content types. However, looking at the interaction plot on the left for 3D there is a slightly different pattern. With 3D the means were relatively high for abstract objects, then decreased for flags, and increased again for art, and followed relatively the same pattern in 2D for females. For males, however, in the 2D plot, the mean for art increased less than it did for females. The pattern of a smaller increase for one content type in the questionnaire data is similar to the pattern observed in the quantitative data but in the quantitative data the male deviation occurs in 3D whereas with the questionnaire data the deviation for males occurs in 2D.

\subsubsection{Subject Comments}

After subjects ranked each carousel, they were asked to comment on the 2D and 3D carousels. Specifically the questionnaire stated: "Please make comments about the effectiveness of 2D versus 3D displays for tasks related to your memory retention". 
Nineteen out of twenty subjects remarked on the effectiveness. The complete comments from each subject can be seen in Appendix H. Some participants made multiple statements in their response; a summary of the frequency of the type of statement can be seen in the Figure 33.

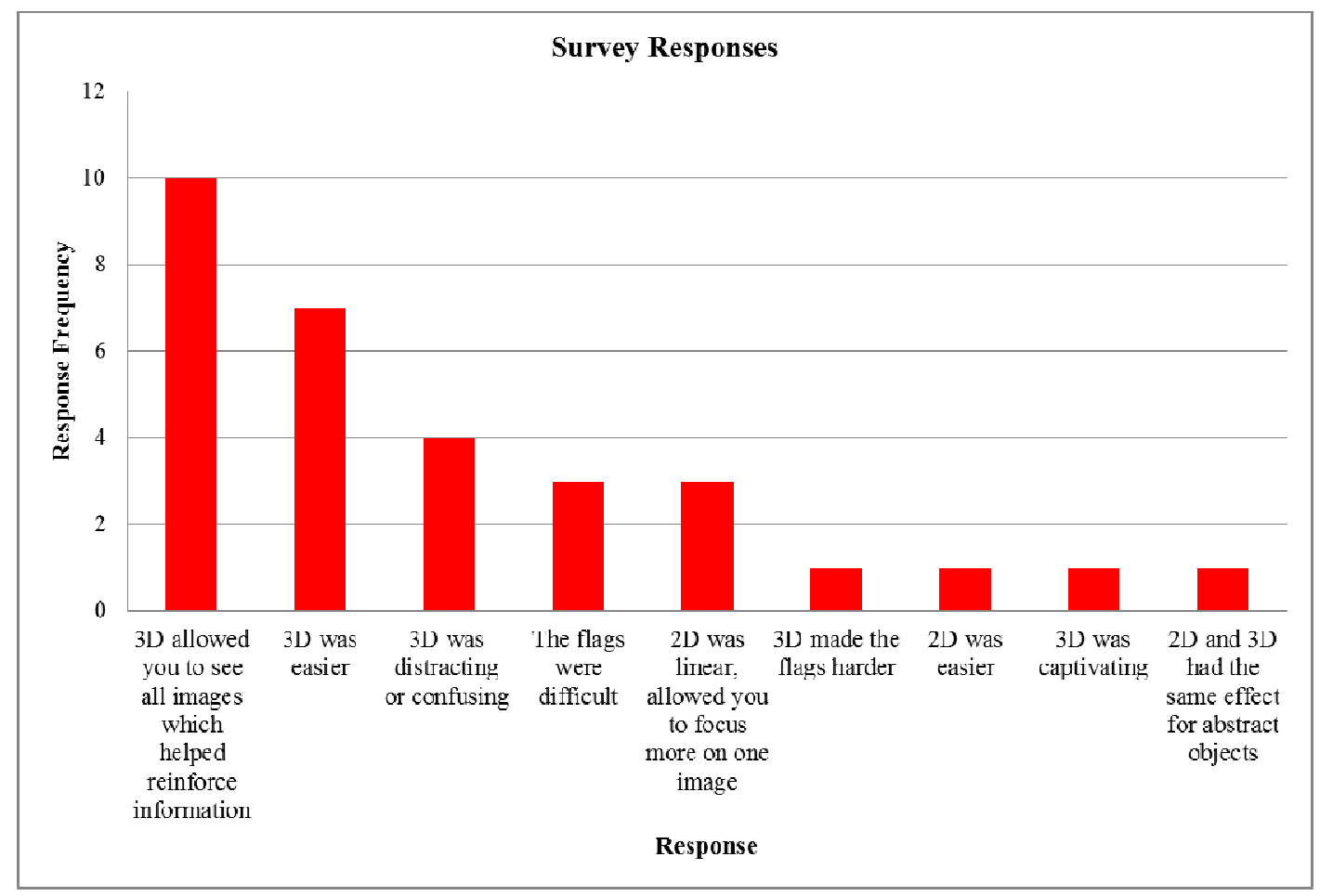

Figure 33 - Summary of Comments from Questionnaire 


\section{CHAPTER 6: DISCUSSION}

The objective of this thesis was to determine if the use of $3 \mathrm{D}$ carousel menus increases retention of information over $2 \mathrm{D}$ menus and to determine if there is a gender effect with these results. Additionally, subjects' perceptions of memory retention with each carousel type were investigated through a questionnaire. A number of findings were revealed from the statistical analysis of the quantitative results and qualitative responses.

\subsection{Quantitative Results}

An ANOVA analysis tested the significance of the main effects (gender, content, and dimension) as well as the first-order and second-order interactions. The results revealed that regardless of the dimension (2D or $3 \mathrm{D})$ content type was a large source of variability, accounting for 26.338 percent of the total variability. The content type of abstract objects had the highest mean of 5.775 correct responses and the other content types of flags and art had lower means of 4.050 and 5.400 correct responses respectively. The flag content type tested associative memory of the subjects and revealed to be more difficult that the other types of content. Dimension and gender did not show to be significant factors. Therefore it can be concluded that when presenting content for memory retention, dimension does not positively or negatively impact the interface's effectiveness for memory retention. Also, there is no gender effect and no first-order interactions.

The second-order interaction effect of Gender vs. Content vs. Dimension was not significant with a P-value of .3360 , but did reveal an interesting pattern in the data. Female subjects had relatively high mean scores for abstract objects, low for flags and 
high for art across both dimensions. Males followed the same pattern for 2D, but with the 3D presentations the increase in the mean from flags to art was much lower, only 4.6 to 4.7 correct responses compared to the female increase of 3.7 to 5.9 correct responses. From the interaction plot of the means for the second-order interaction there appears to be a difference for males when moving from 2D to 3D across the different content types. This difference, in addition to the significance of the factor content type may indicate the presence of confounding variables such as subjects' attitudes toward a content type or previous knowledge of a content type. In order to assess if there were other confounding factors such as learning or fatigue, a simple regression model was run for each subject's results to test for trends in performance over all twelve trials (six training and six experimental trials). There were no significant slopes, indicating that there was no learning (positive improvement in score) or fatigue (decrease in score).

While content level was a large source of variability and other interesting patterns were observed in the second-order interaction charts, the overarching finding from the quantitative results is that there is no indication of the effectiveness of $3 \mathrm{D}$ over $2 \mathrm{D}$ for memory retention purposes.

\subsection{Qualitative Results}

The qualitative results differed in pattern to the quantitative results in that content was not a large source of variability. Although not significant, subjects scored the carousels with flags, regardless of dimension (2D or 3D), lower than the carousels with other content types. This lower score for flags was consistent with subject's lower performance on the assessments for carousels with flags. This low ranking was also 
reflected in the comments made by multiple subjects stating that the flags were difficult, and in one case even stating that the 3D presentation made the flags more difficult.

The qualitative results also showed that Content*Dimension was a large source of variability in the data, accounting for 18.677 percent of the total variability. From the interaction plot of Content*Dimension it can be observed that subjects gave higher rankings for 3D across all content types.

Overall the ratings were higher for 3D regardless of gender and content but not significantly (P-value of 0.8460). From the subjects' comments, most subjects believed that the 3D interfaces aided their retention more than the 2D interfaces. Many of the subjects believed the reason why $3 \mathrm{D}$ aided retention more was because they were able to see all of the images, allowing them to review images again. From the qualitative results, many subjects viewed 3D as a benefit to aiding their retention, although this was not supported through the statistical analysis.

Additional questionnaire comments revealed that some subjects believed the linear nature of the 2D display was beneficial in aiding retention and allowed users to focus more on one image. Comments suggested that the 2D display was capable of showing more information for one image whereas the 3D display is better at showing general characteristics of multiple images. The linearity of the 2D display was also thought to be more familiar by one subject. While these comments revealed the possible advantages of 2D menus, they were outnumbered by the positive comments regarding the 3D menus. 


\section{CHAPTER 7: CONCLUSION AND FUTURE WORK}

\subsection{Conclusion}

There is an increasing presence of 3D menus in UI design; consequently there is a growing interest in the field of HCI to study the effects 3D menus have on cognitive processes. Multiple application areas for 3D menus have been identified where memory retention is a critical success factor, but little research has currently been done in the area of memory retention for 3D UIs. This thesis compared 2D and 3D menus in user interface design and investigated the impact on memory retention across multiple content types.

The results of this experiment showed that dimension did not reveal to be a significant factor in the retention of information. Therefore, in application areas where retention of information is a critical success factor, it can be concluded that the use of a 3D menu neither promotes nor degrades memory retention. Although menu retention was not affected by changing dimension, several subjects' responses demonstrated that the menu dimension they perceived to better aid retention was 3D. Based on these findings it is recommended that user interface designers utilize whichever menu type, $2 \mathrm{D}$ or $3 \mathrm{D}$, is most suitable for the other interface design considerations and criteria since there is not significance in the quantitative or qualitative results regarding menu dimension.

It is important to note that the boundaries chosen for this experiment limited the focus to studying memory retention with two different menu dimensions for young, novice users. Within the boundaries chosen, the factors of gender and dimension did not impact memory retention. While the results of this thesis have contributed to the understanding of the cognitive impacts of using 3D menus, there still remains a great opportunity for research in the area of 3D. 


\subsection{Future Work}

The results of this thesis have demonstrated that within the boundaries chosen, displaying various types of content in $3 \mathrm{D}$ versus $2 \mathrm{D}$ does not make a significant difference on memory retention. Although this work is limited to memory retention there is an interest in the other cognitive processes that may be affected by presenting information in 3D, for instance identification through scanning, and logical operations.

There are still a number of questions that need to be addressed regarding the use of 3D menus, specifically relating to different cognitive processes and applications. For each of the following questions an experiment can be designed to investigate the effects of 2D and 3D UIs:

- Are 3D UIs better for image scanning than 2D UIs?

- Are 3D UIs better for logical operations than 2D UIs?

- Are 3D UIs more suitable that 2D UIs as visualization tools for big data? Within each of the questions listed above the following factors should also be investigated:

- Is subject experience level a significant factor?

- Is subject age a significant factor?

The increasing use of 3D effects in UI design for a variety of applications has opened the field of research concerned with the impact that moving from $2 \mathrm{D}$ to $3 \mathrm{D}$ presentations of information has on cognitive processes. This thesis is a step toward expanding the knowledge in the field of HCI related to 3D UIs. This research was limited to the factors selected in the experimental design. Future researchers should investigate 
the effect that 3D UIs have on other cognitive processes and design experiments that explore other factors such as subject experience level and age. 


\section{References}

1. Alpcan, T., Bauckhage, C., \& Kotsovinos, E. "Towards 3D Internet: Why, What, and How?" Proc. of 2007 International Conference on Cyberworlds. N.p.: IEEE, n.d. 95-99. Print.

2. Björkskog, C., Jacucci, G., Lorentin, B., \& Gamberini, L.: Mobile implementation of a web 3D carousel with touch input. In: Proc. of the 11th International Conference on Human-Computer Interaction with Mobile Devices and Services, pp. 1-4. ACM, New York (2009).

3. Chalmers, Patricia A. "The Role of Cognitive Theory in Human-computer Interface.” Computers in Human Behavior, 19.5 (2003): 593-607.

4. “Cover Flow.” Wikipedia. Wikimedia Foundation. Web. 15 May 2013.

5. Dachselt, R \& Hübner, A. Three-dimensional menus: A survey and taxonomy, Computers \& Graphics, Volume 31, Issue 1, January 2007, Pages 53-65.

6. Gasston, P. "Adventures In The Third Dimension: CSS 3D Transforms." Smashing Magazine. N.p., 6 Jan. 2012. Web.

7. Jako, J., Salvendy, G, \& Koubek, R. "Modeling of Menu Design in Computerized Work.” Interacting with Computers 7.3 (1995): 304-30. Web.

8. “Javascript 3D Carousel with Scriptaculous.” WebAppers. 15 May 2013.

9. Kahn, S., and Kahn, A. "Showcase of Creative Navigation Menus: Good and Bad Examples.” Smashing Magazine.19 Apr. 2011.

10. Kiger, J. "The Depth/Breadth Tradeoff in the Design of Menu-Driven Interfaces," International Journal of Man-Machine Studies (20) 1984, pp 201-213. 
11. Kim, K., Jacko, J., \& Salvendy, G. (2011). Menu design for computers and cell phones: Review and reappraisal. International Journal of Human-Computer Interaction, 27(4), 383-404.

12. Kim, N., Kim, G., Park, C., Lee, I., \& Lim, S. (2000). Multimodal Menu Presentation and Selection in Immersive Virtual Environments. Proceedings of IEEE Virtual Reality. 281-288.

13. Kim, K., Proctor, R., \& Salvendy, G. Comparison of 3D and 2D menus for cell phones, Computers in Human Behavior, Volume 27, Issue 5, September 2011, Pages 2056-2066, ISSN 0747-5632, 10.1016/j.chb.2011.04.014.

14. Koutstaal, W., Chandan, R., Jackson, E., Prince, S. Cendan, D., \& Schacter, D. "False Recognition of Abstract Versus Common Objects in Older and Younger Adults: Testing the Semantic Categorization Account.” Journal of Experimental Psychology. Learning, Memory, and Cognition, 29.4 (2003): 499-510.

15. Molina, J., Gonzalez, P., Lozano, M., Montero, F., \& Lopez-Jaquero, V. (2003). Bridging the gap: Developing 2D and 3D user interfaces with the IDEAS methodology. In Proceedings of 10th international conference on design, specifications, and verification of interactive systems DSV-IS 2003 (pp. 303-315).

16. Norman, K. The Psychology of Menu Selection. Ablex Publishing Corporation, Norwood, NJ, 1991.

17. Paap, K. \& Roske-Hofstrand, R. (1988) 'Design of menus' in Helander, M (Ed). Handbook of Human-Computer Interaction. Elsevier, 205-235. 
18. Patterson, D. "3D Space: Using Depth and Movement for Selection Tasks." Proceedings of the Twelfth International Conference on 3D Web Technology. Web 3D '07. New York: ACM, 2007. 147-55.

19. Read, A., Tarrell,A., \& Fruhling, A. "Exploring User Preference for the Dashboard Menu Design.” Proceedings of the 42nd Hawaii International Conference on System Sciences - 2009.Print.

20. Robertson, G., Card, S., \& Mackinlay, J. (1993). Information visualization using 3D interactive animation. Communications of the ACM, 36(4), 57-71.

21. Robertson, G., Czerwinski, M., Larson, K., Robbins, D., Thiel, D., \& van Dantzich, M. (1998). Data mountain: Using spatial memory for document management. Proceedings of the 11th Annual ACM Symposium on User Interface Software and Technology,153-162.

22. Sebrechts, M., Cugini, J., Laskowski, S., Vasilakis, J., \& Miller, M. (1999). Visualization of search results: a comparative evaluation of text, 2D, and 3D interfaces. In Proceedings of the 22nd annual international ACM SIGIR conference on Research and development in information retrieval (pp. 3-10). Berkeley, CA: ACM.

23. Simon, S. "The Impact of Culture and Gender on Web Sites: An Empirical Study." Database for Advances in Information Systems, 32.1 (2201):18.

24. Sundar, S., Bellur, S., Oh, J., \& Jia, H. "Calls for Interaction: The More the Better? User Experience of 3D Carousel and Additional Interaction Techniques. “Human Computer Interaction- INTERACT 20116949 (2011): 487-90. Web. 
25. Wang, S., Poturalski, M., \& Vronay, D.: Designing a generalized 3D carousel view. In: CHI 2005 Extended Abstracts on Human Factors in Computing Systems, pp. 2017-2020. ACM Press, New York (2005).

26. Wickens, C., Gordon, S. and Liu, Y. An Introduction to Human Factors Engineering. Upper Saddle River, NJ: Pearson Prentice Hall, 2004. Print. 
APPENDIX A: Image Sets and Assessment Questions

\section{Abstract Objects Set 1 - Images}
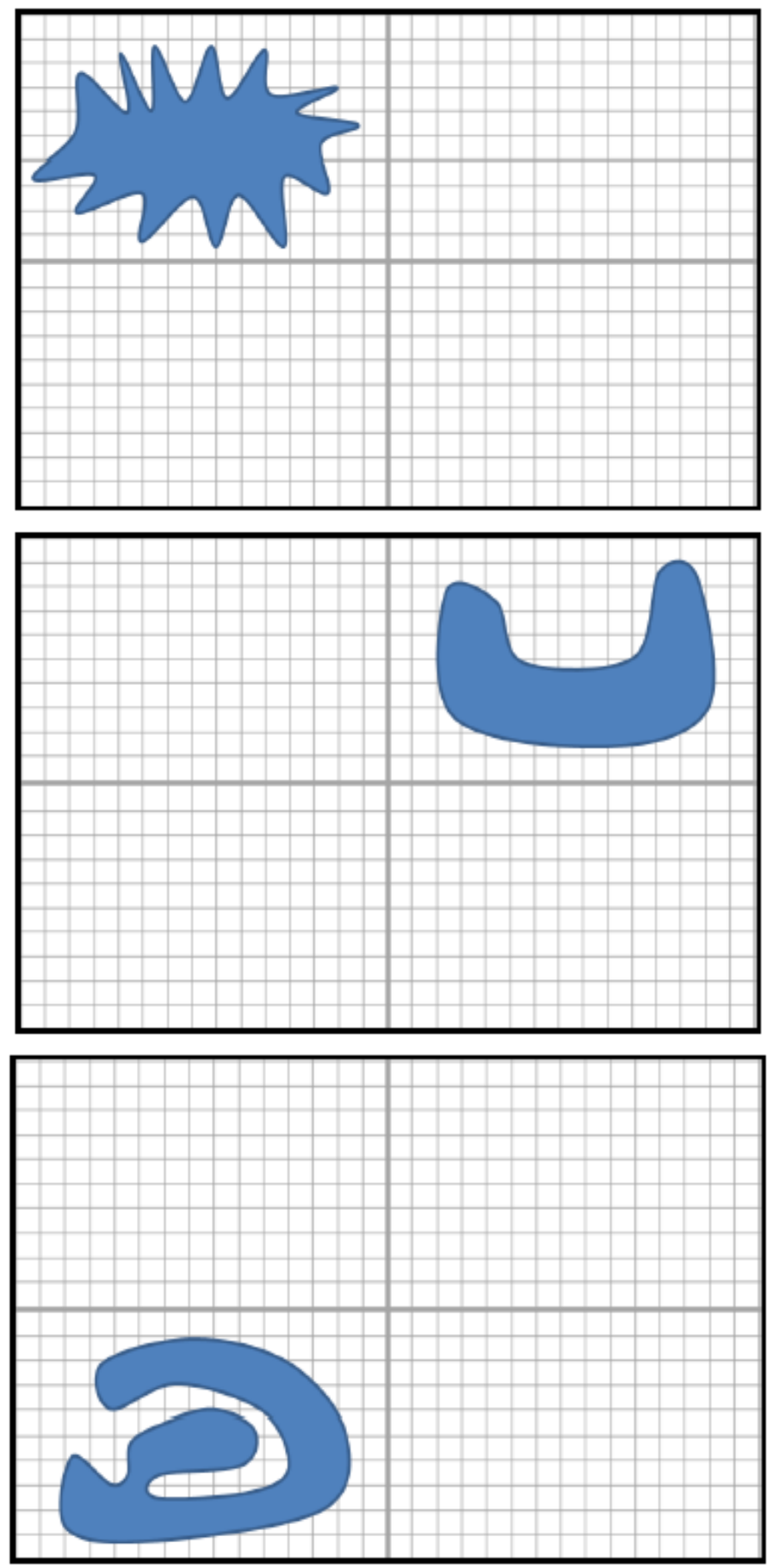

Page 62 

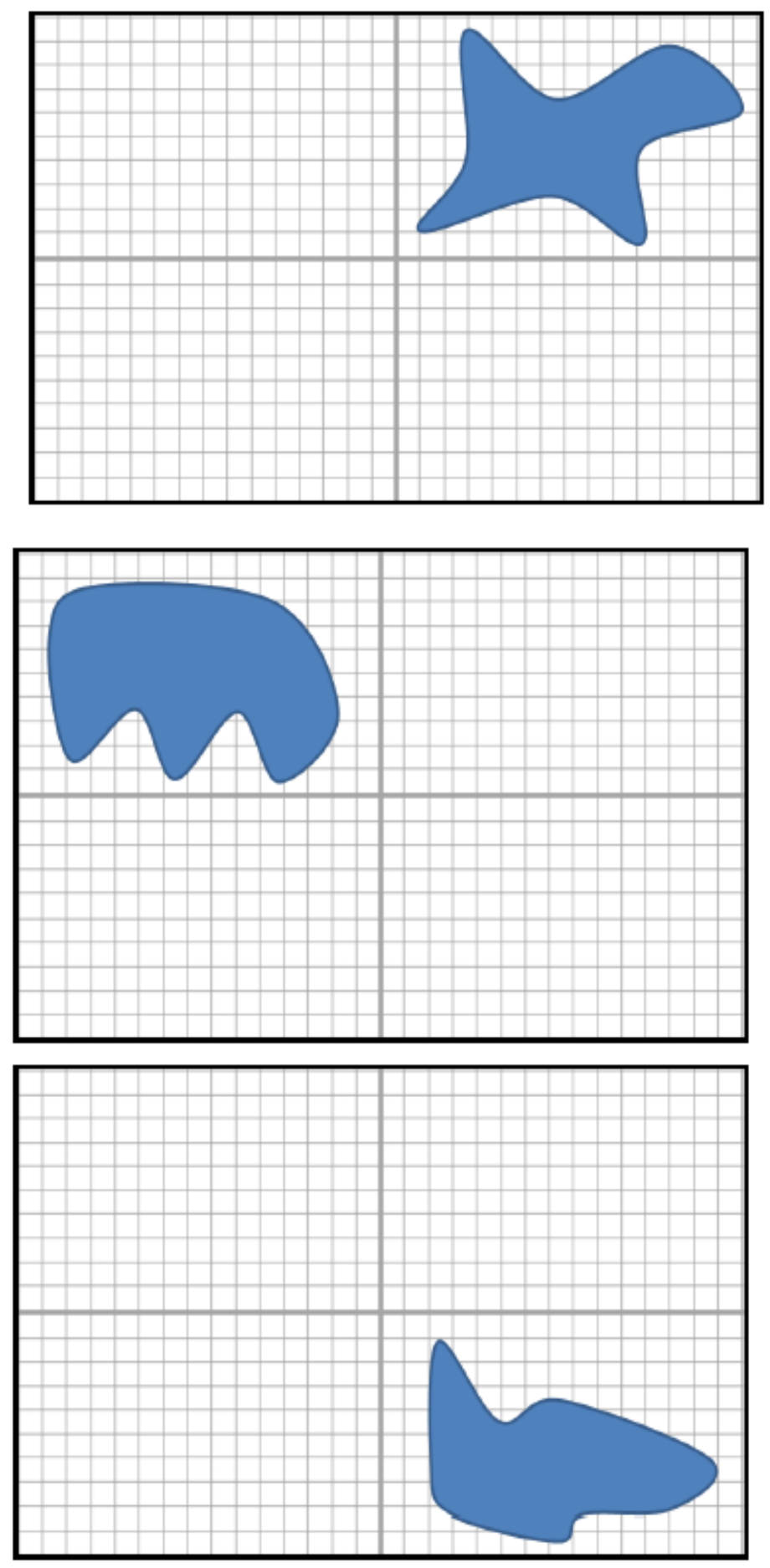

Page 63 


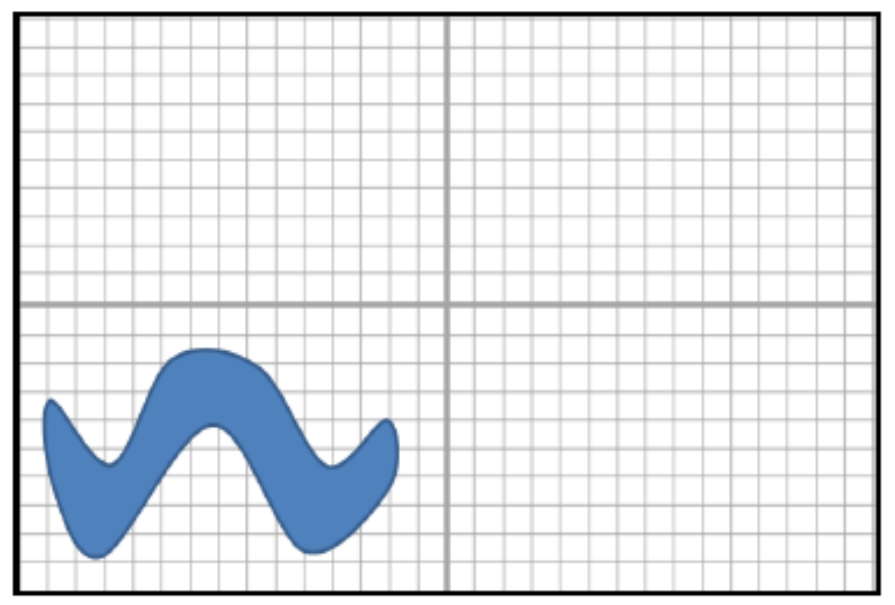

\section{Abstract Objects Set 1 - Assessment}

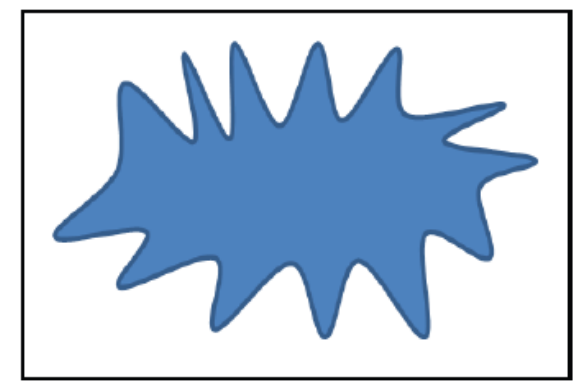

Which quadrant did the image appear in?

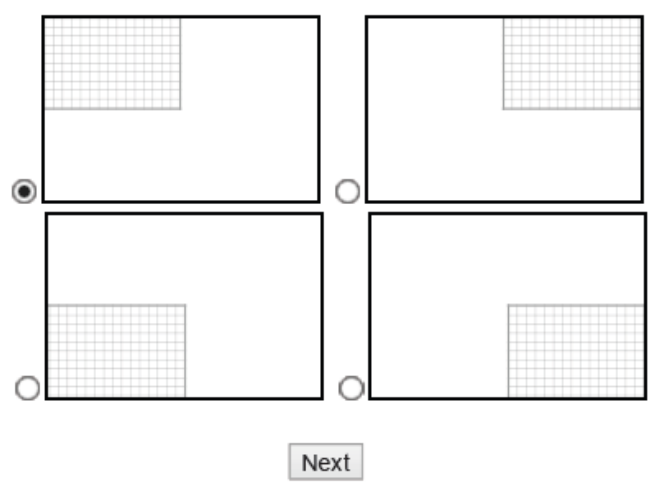

Page 64 


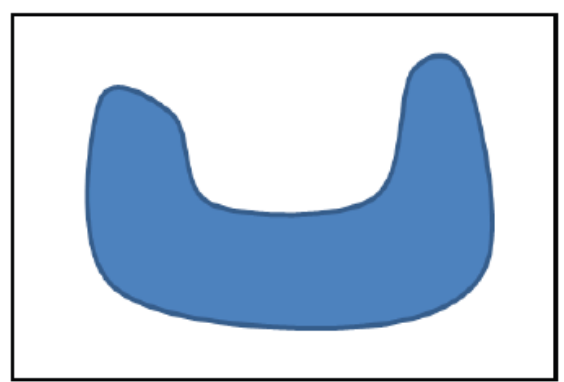

Which quadrant did the image appear in?

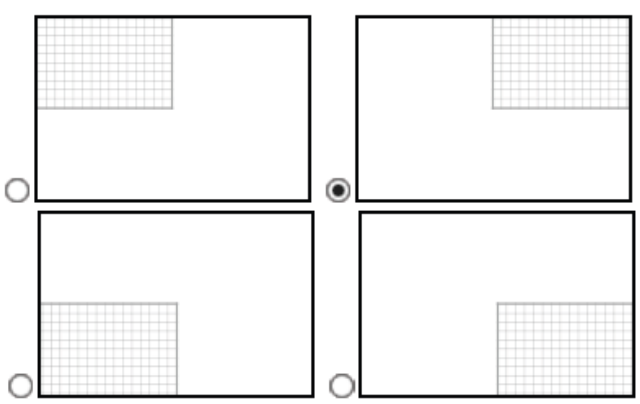

Next

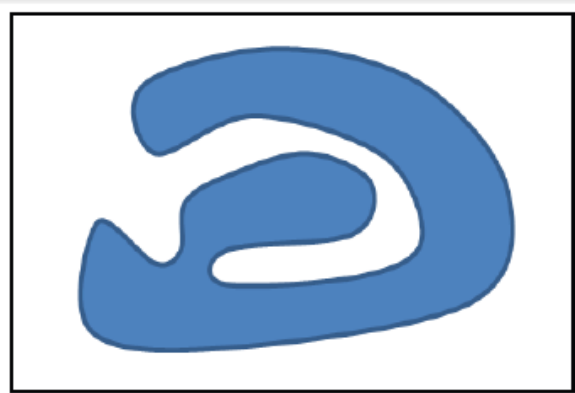

Which quadrant did the image appear in?

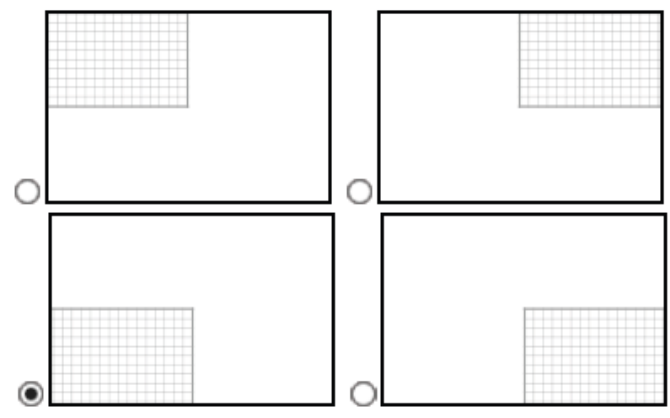

Next

Page 65 


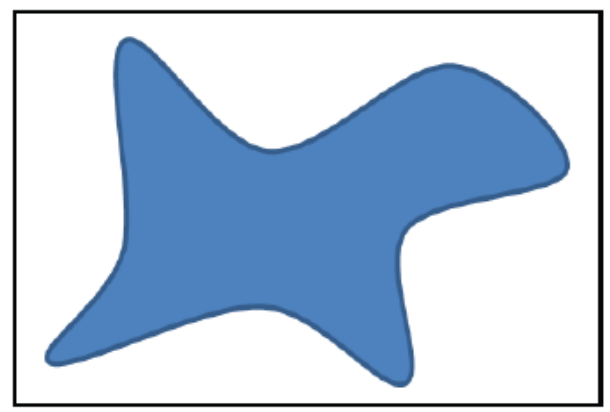

Which quadrant did the image appear in?
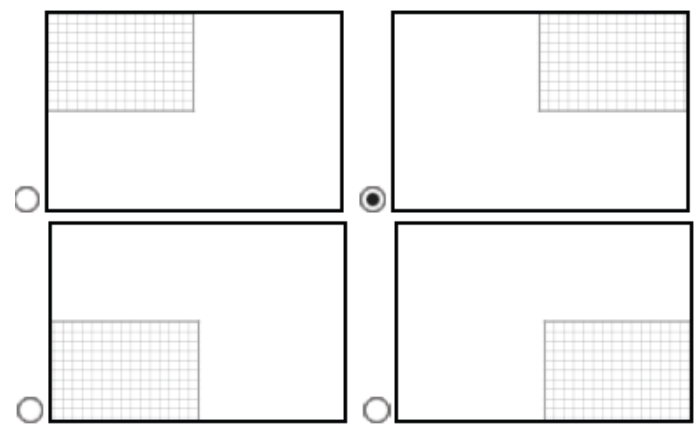

Next

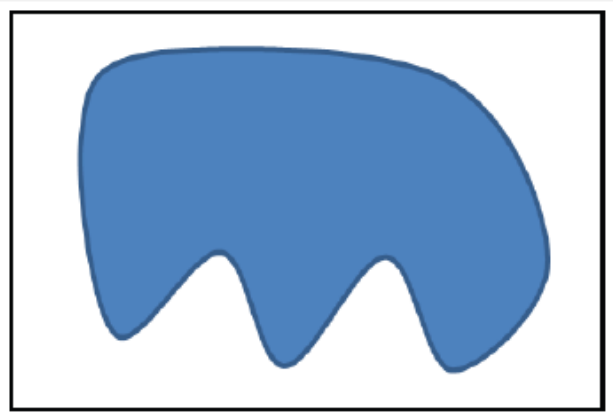

Which quadrant did the image appear in?

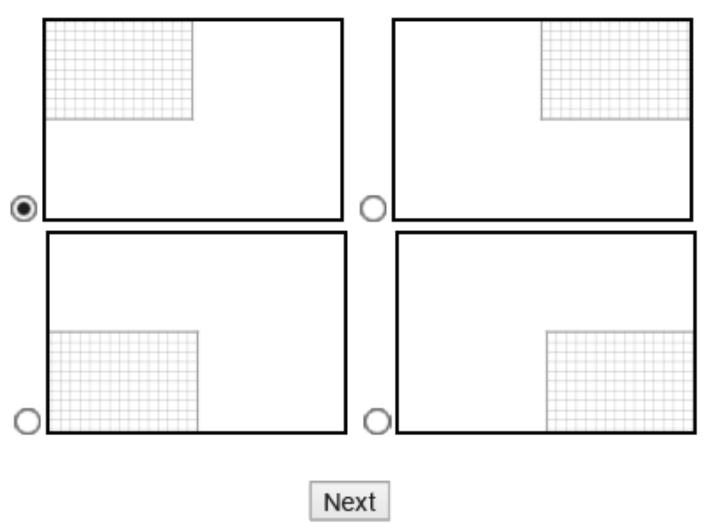

Page 66 


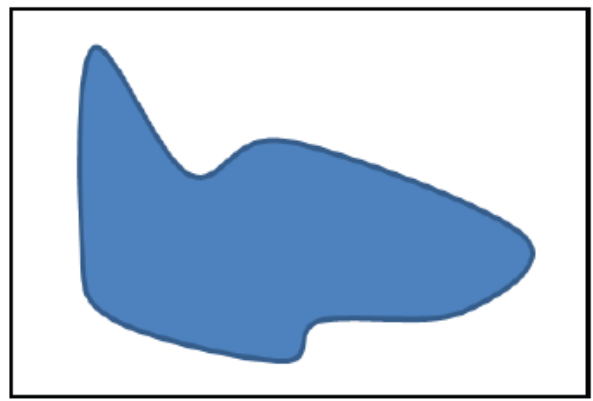

Which quadrant did the image appear in?
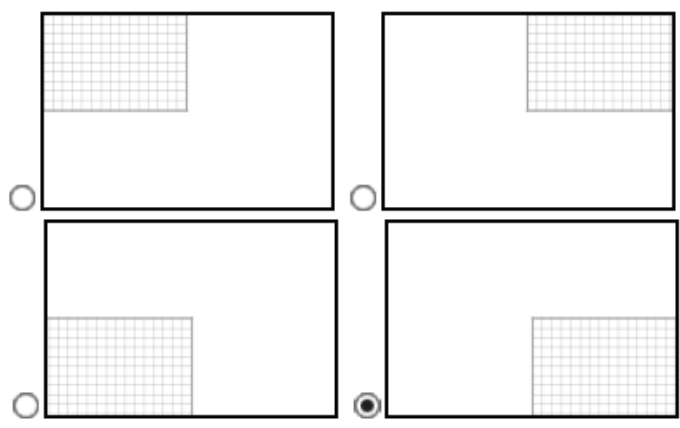

Next

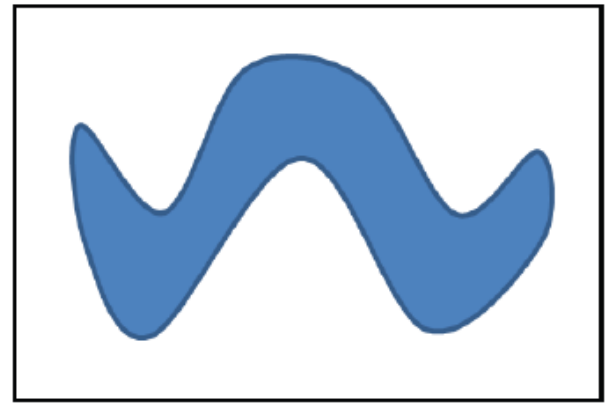

Which quadrant did the image appear in?
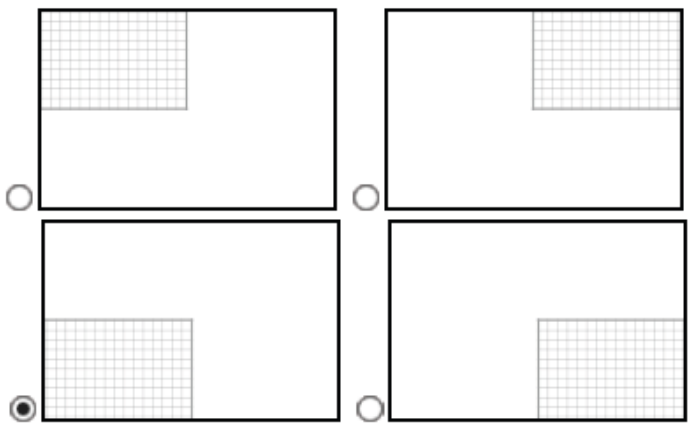

Next

Page 67 
Abstract Objects Set 2 - Images
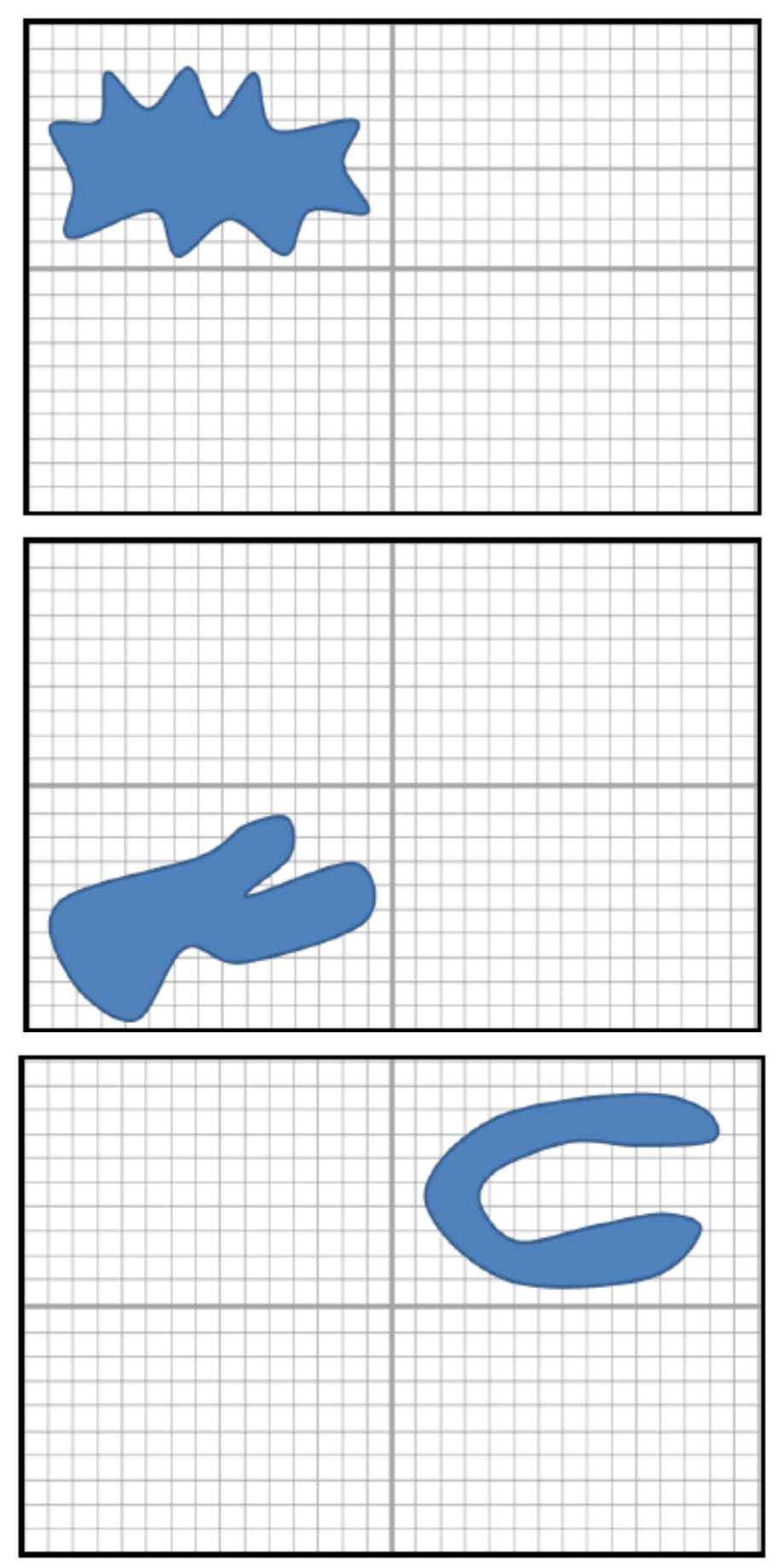

Page 68 

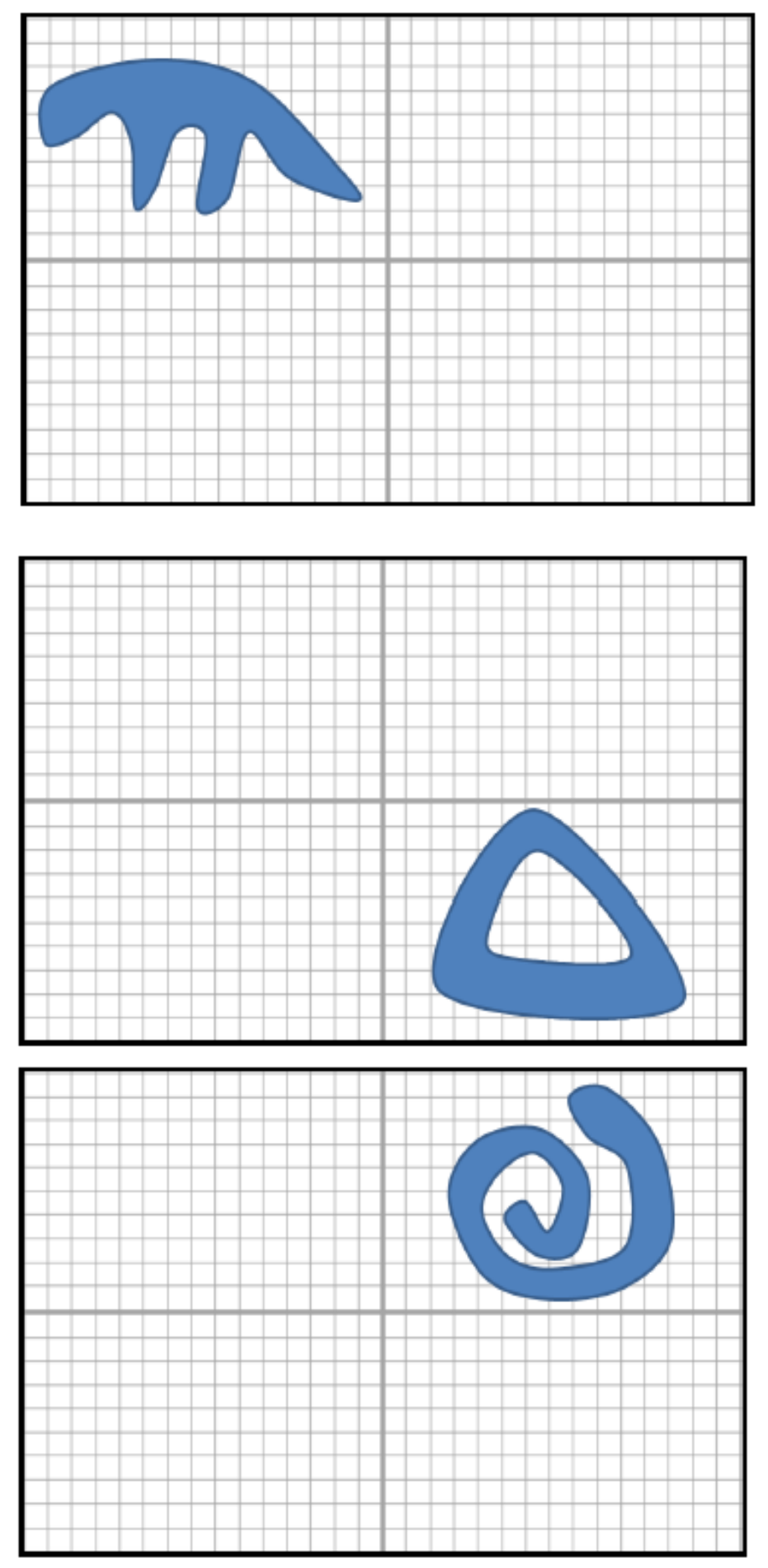

Page 69 


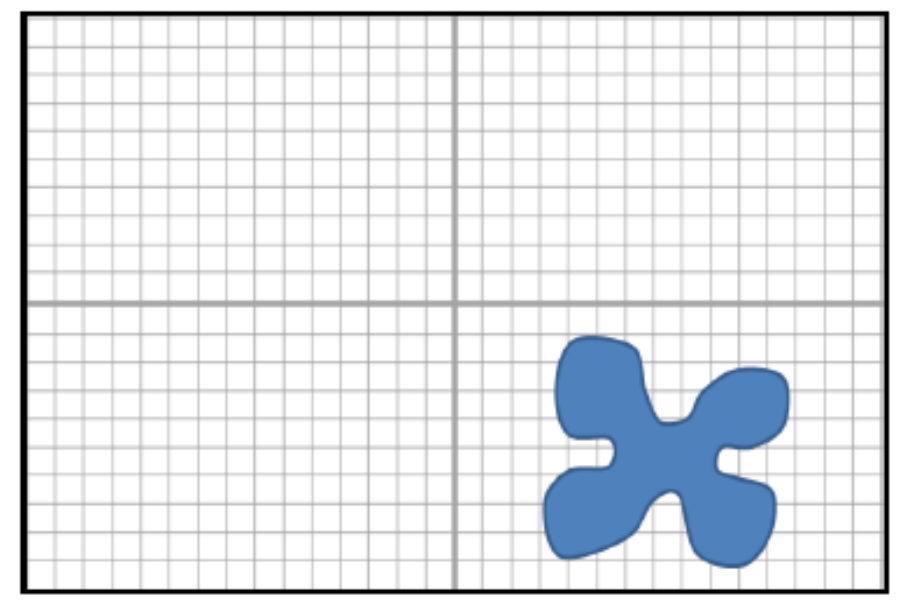

Abstract Objects Set 2 - Assessment

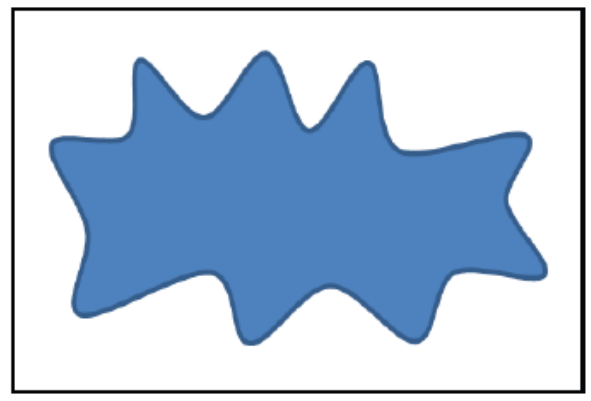

Which quadrant did the image appear in?
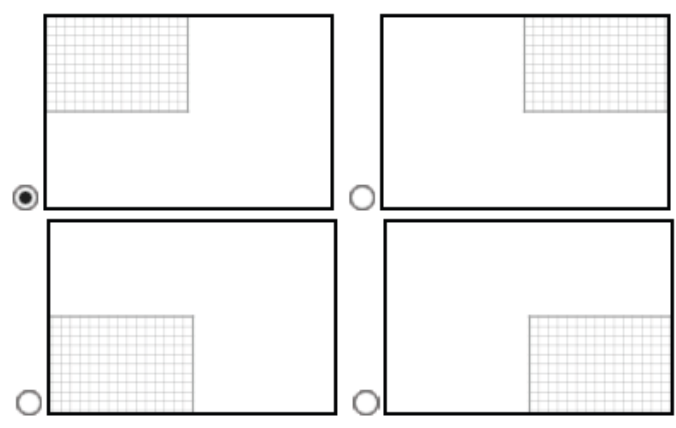

Next

Page 70 


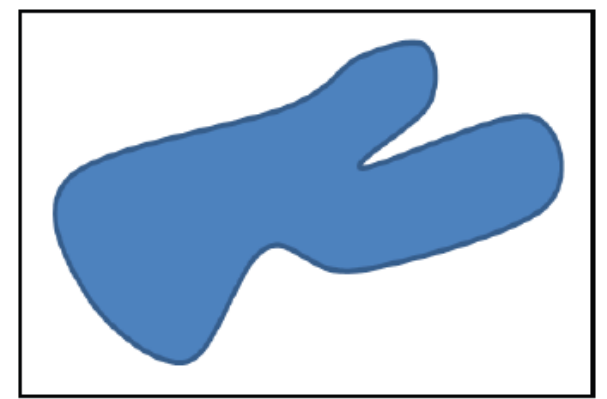

Which quadrant did the image appear in?

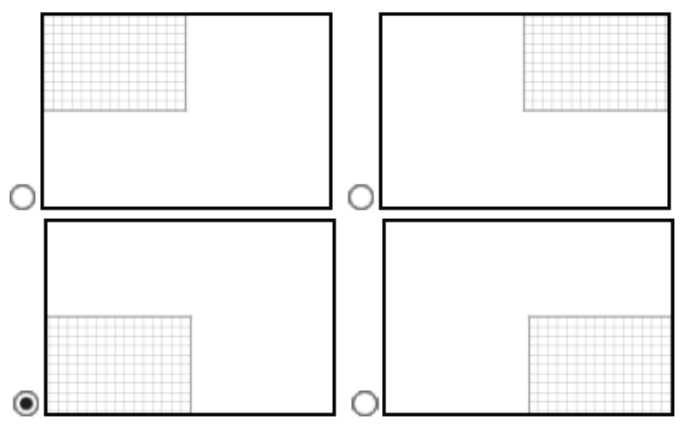

Next

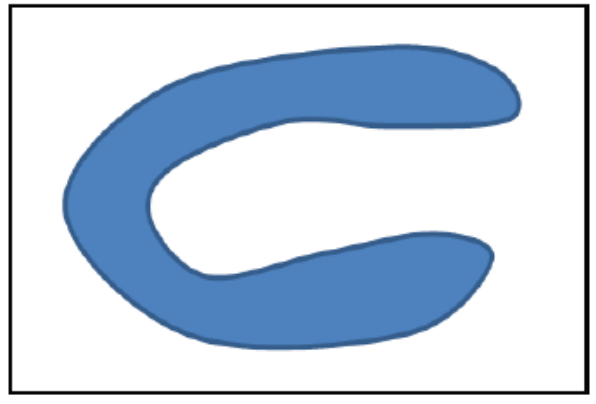

Which quadrant did the image appear in?
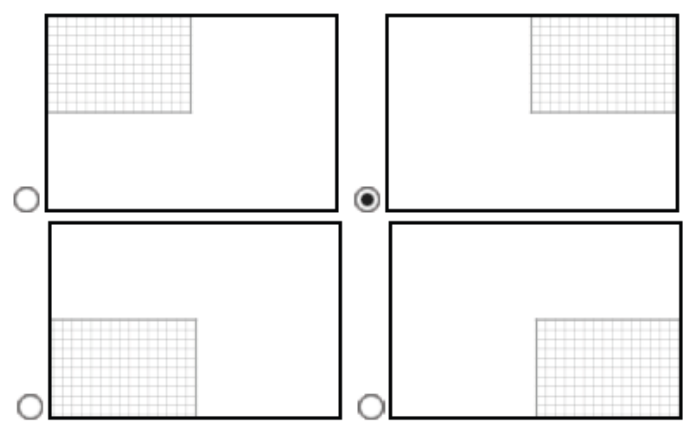

Next

Page 71 


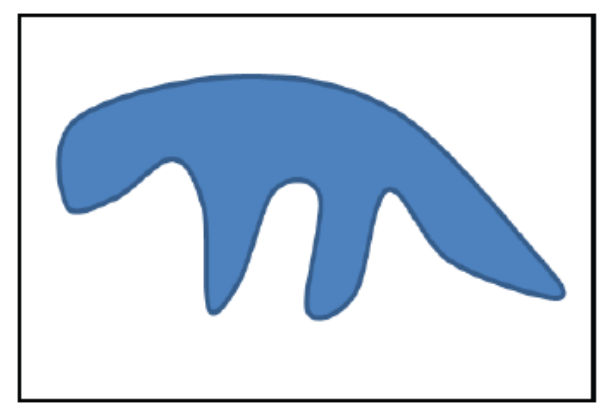

Which quadrant did the image appear in?

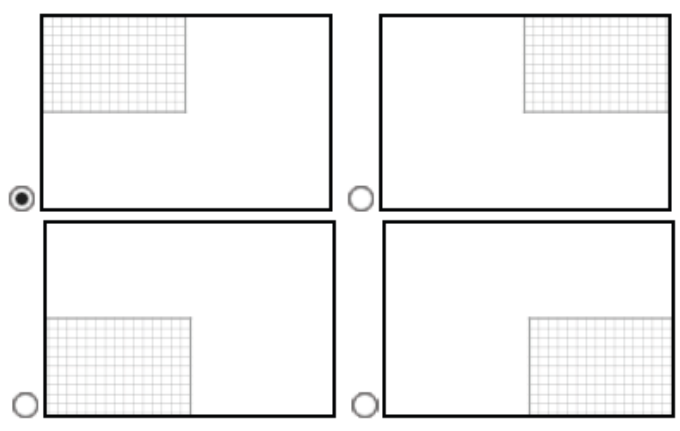

Next

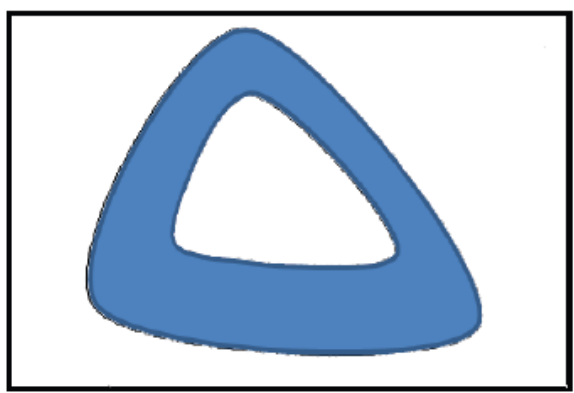

Which quadrant did the image appear in?
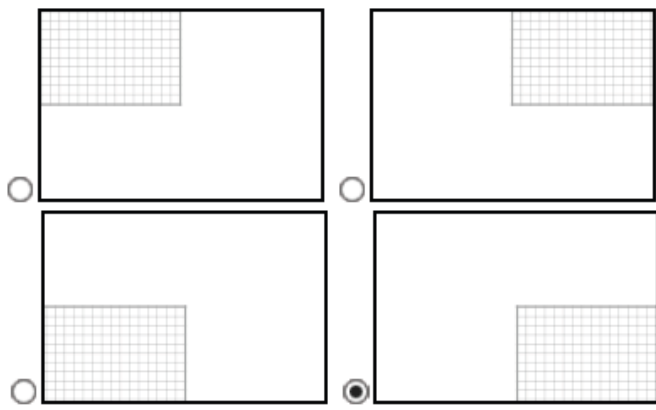

Next

Page 72 


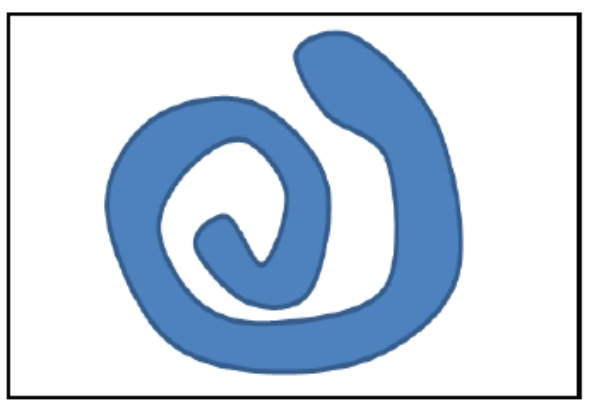

Which quadrant did the image appear in?
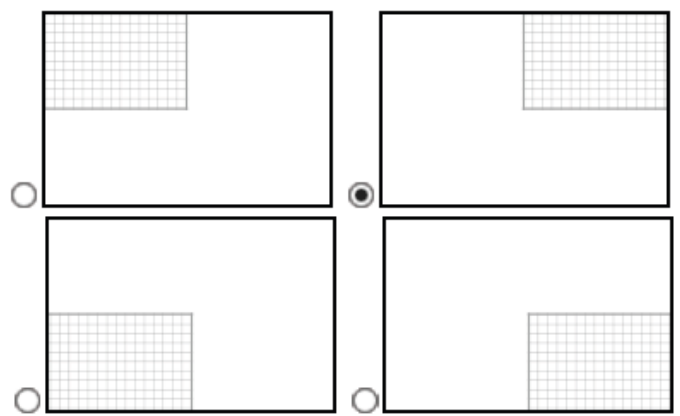

Next

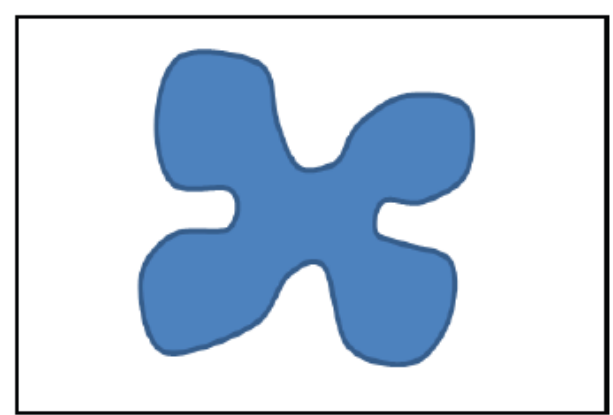

Which quadrant did the image appear in?
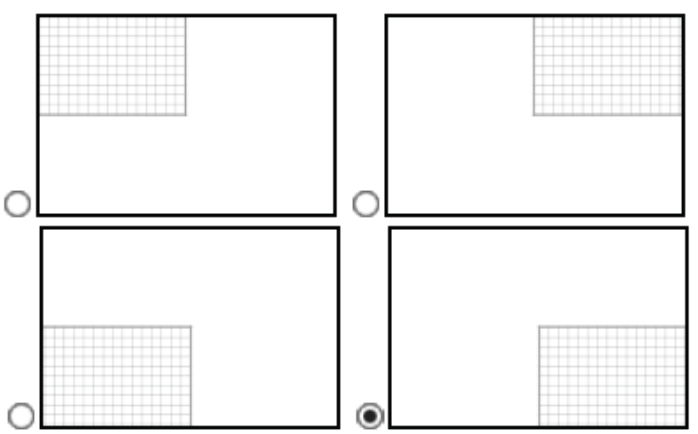

Next

Page 73 
Abstract Objects Set 3 - Images
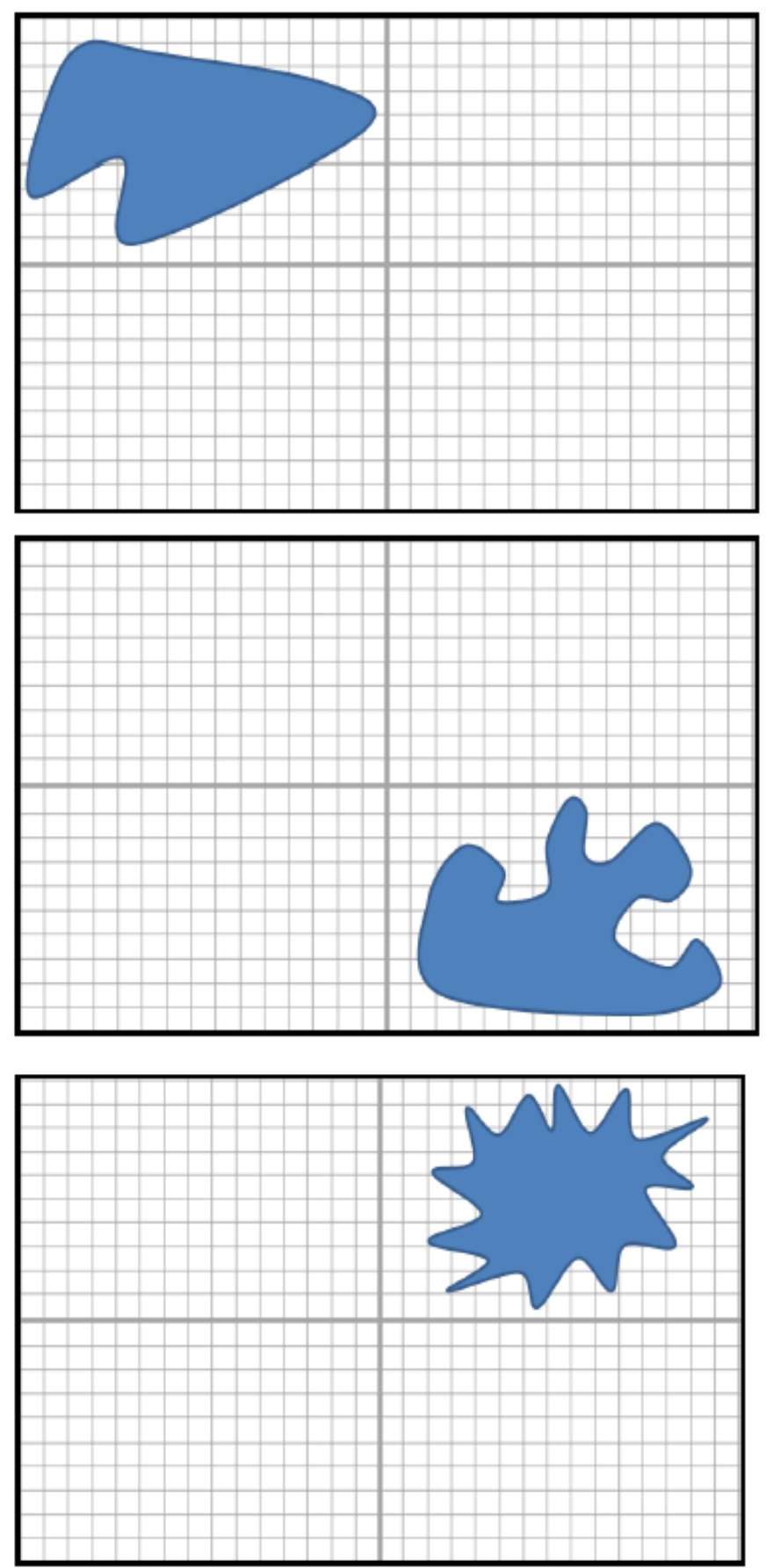

Page 74 

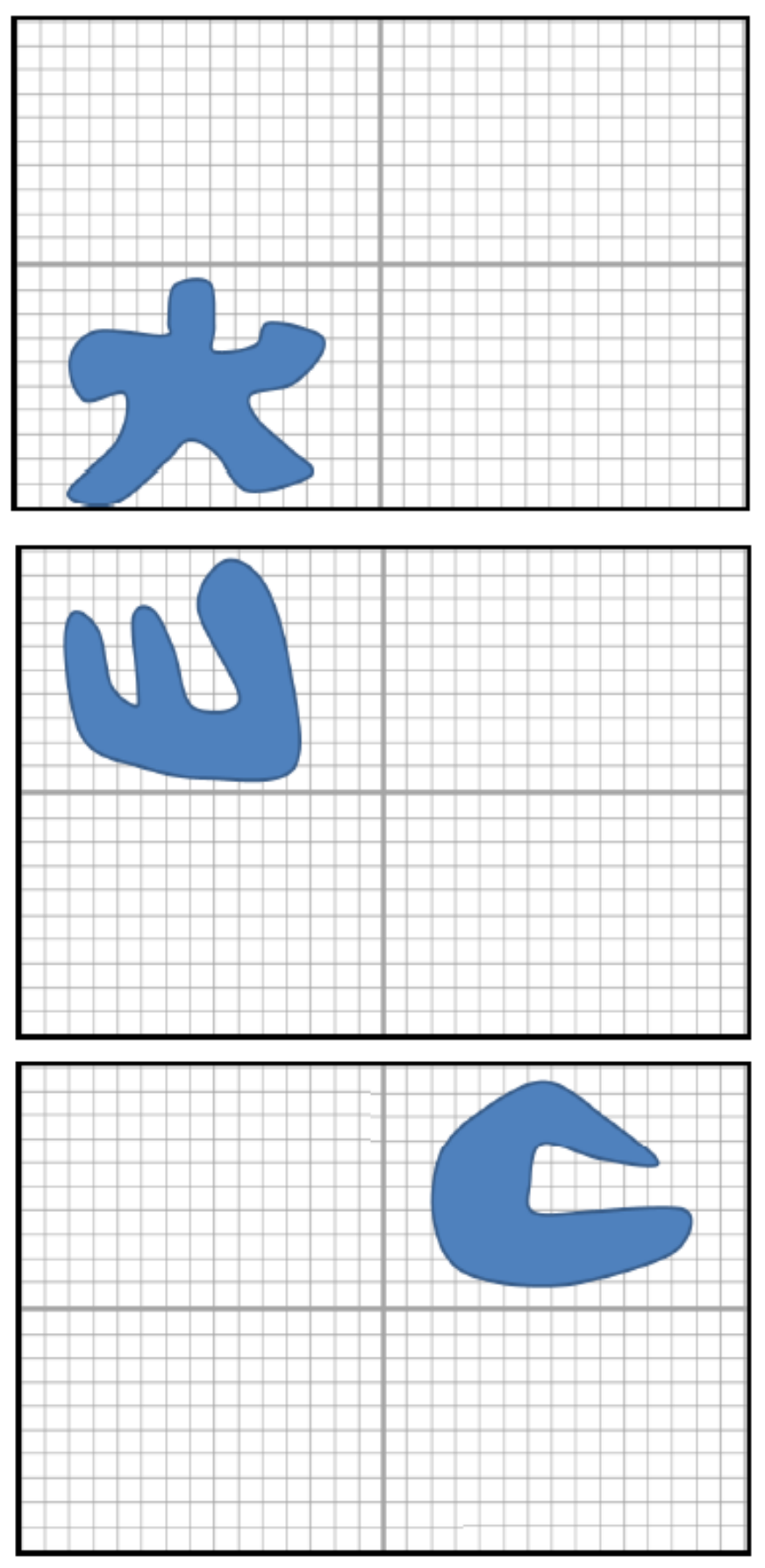

Page 75 


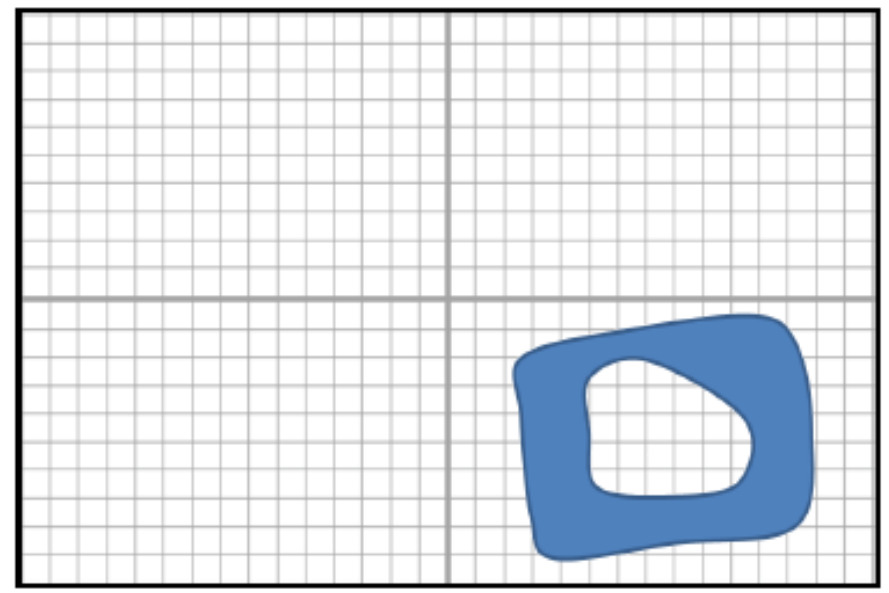

Abstract Objects Set 3 - Assessment

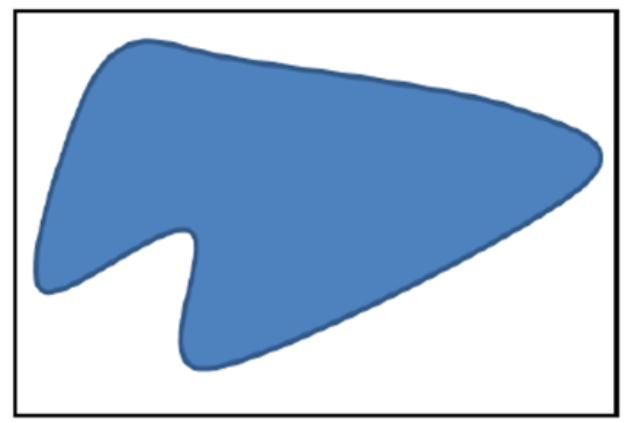

Which quadrant did the image appear in?

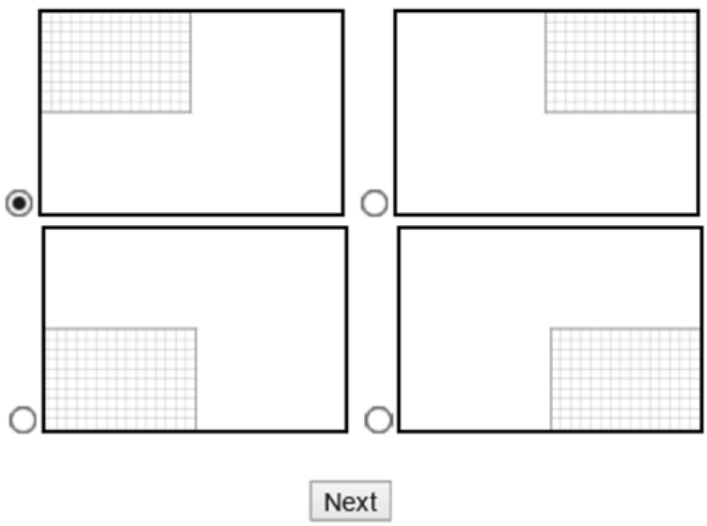

Page 76 


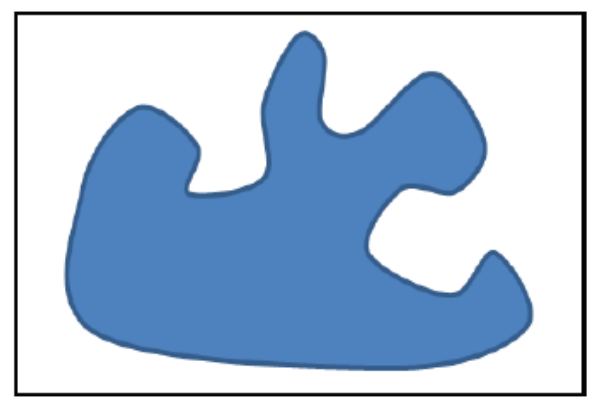

Which quadrant did the image appear in?
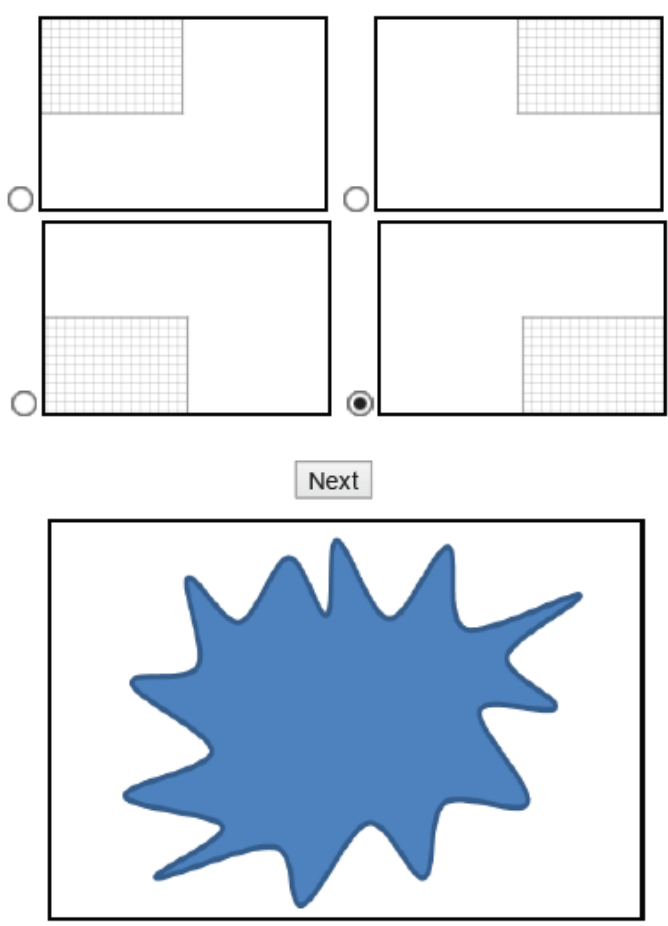

Which quadrant did the image appear in?

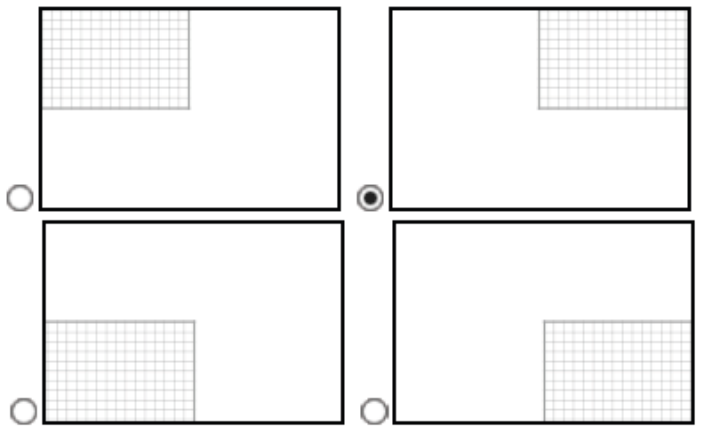

Next

Page 77 


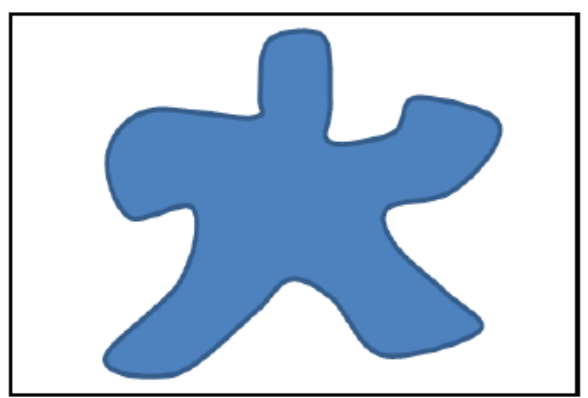

Which quadrant did the image appear in?

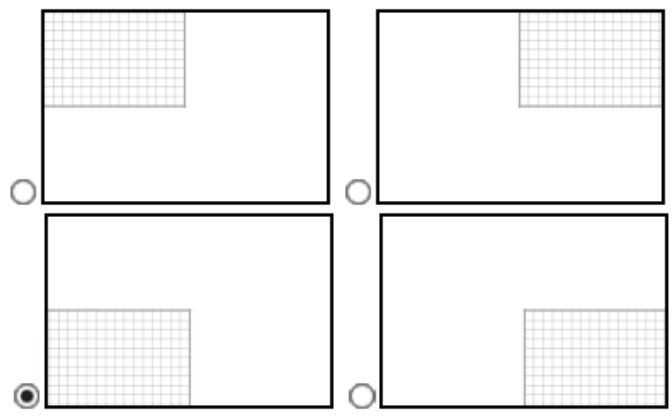

Next

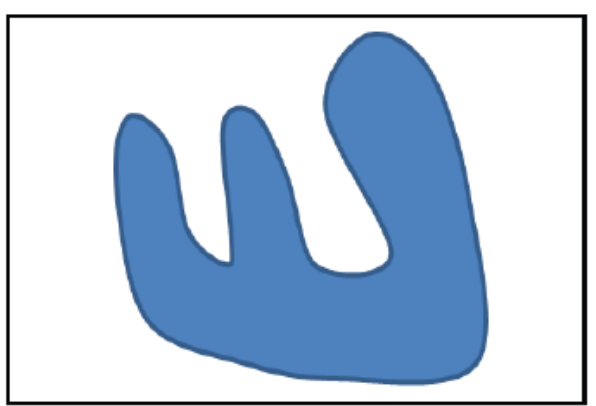

Which quadrant did the image appear in?

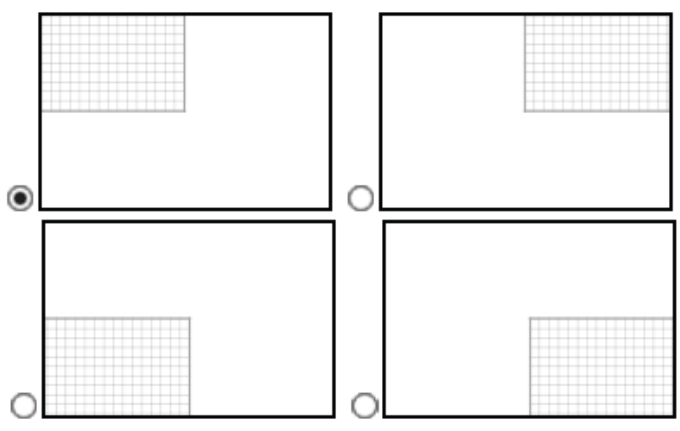

Next

Page 78 


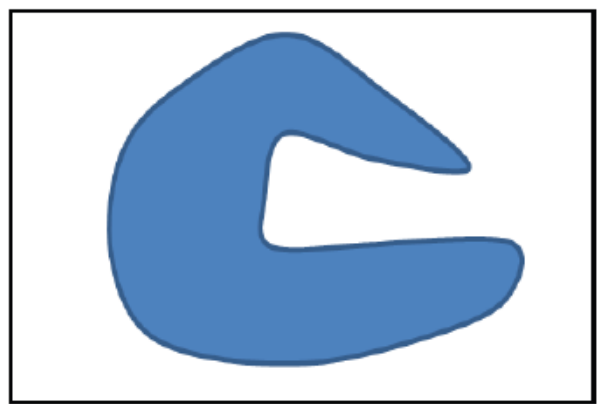

Which quadrant did the image appear in?
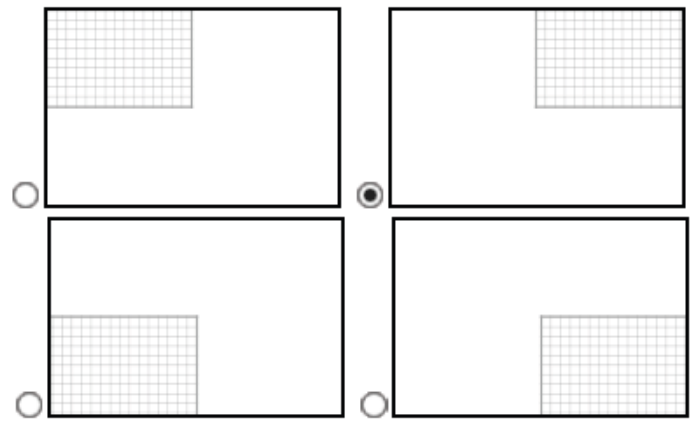

Next

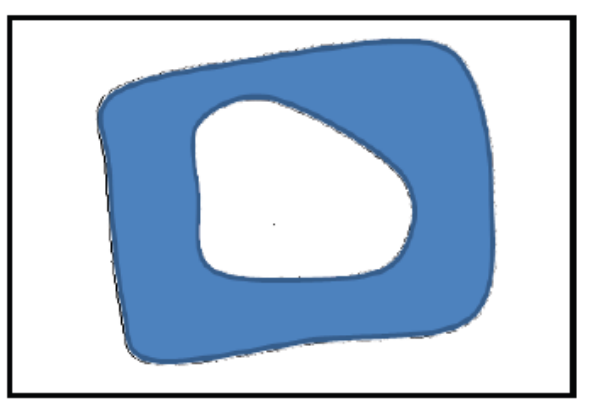

Which quadrant did the image appear in?

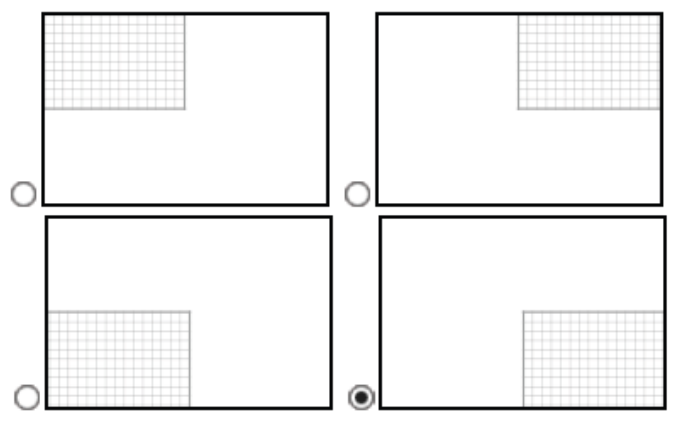

Next

Page 79 


\section{Abstract Objects Set 4 - Images}
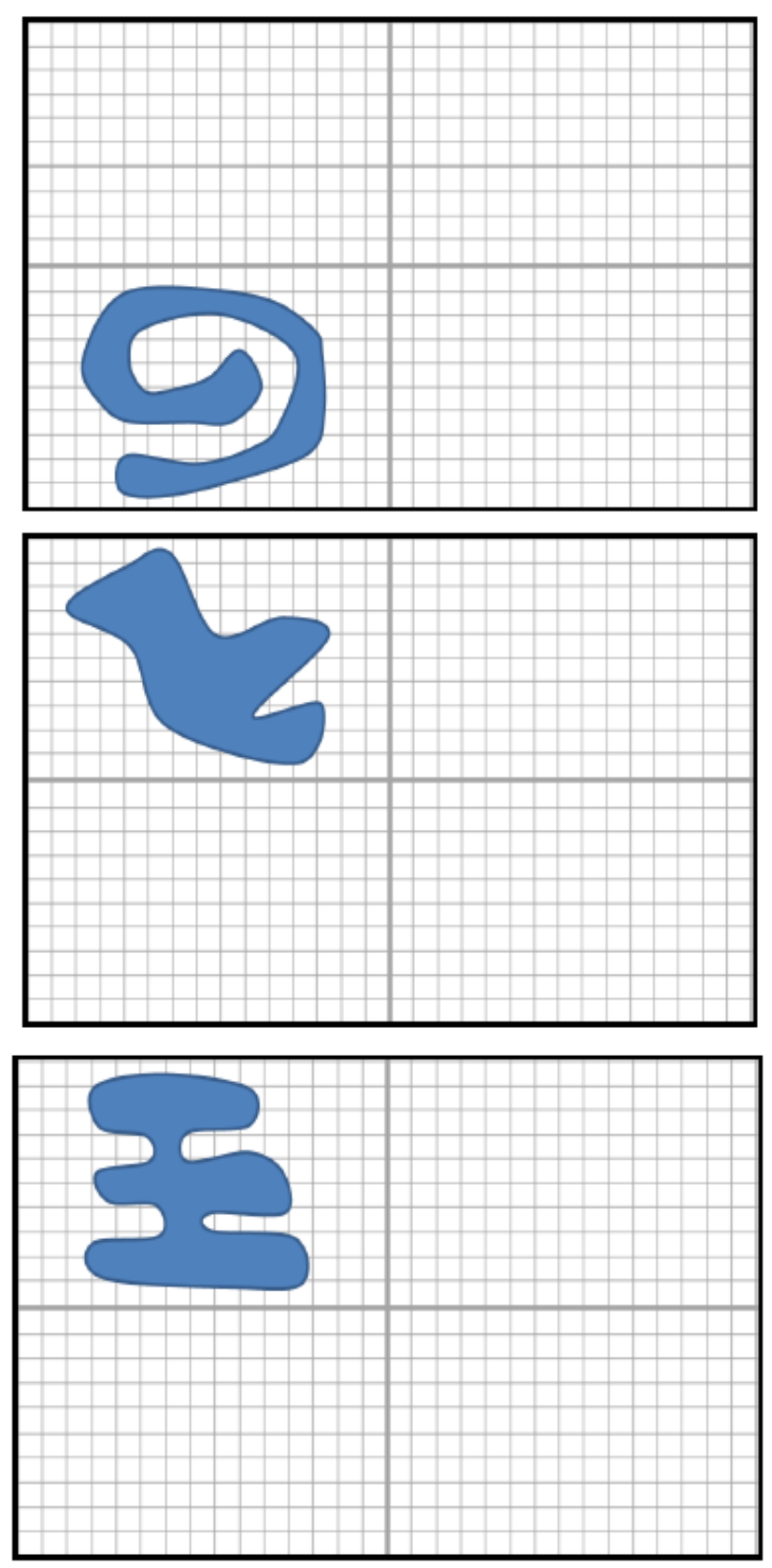

Page 80 

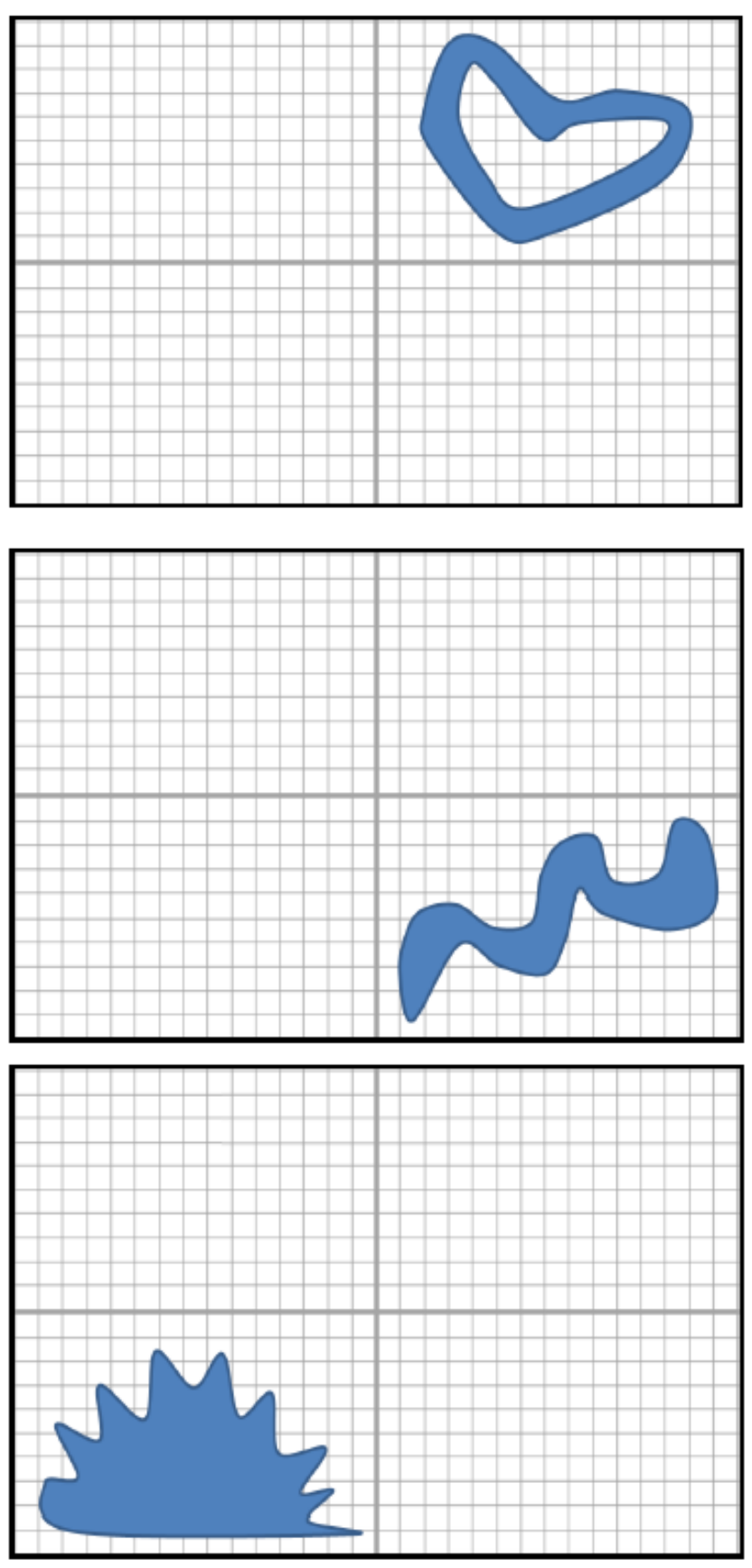

Page 81 


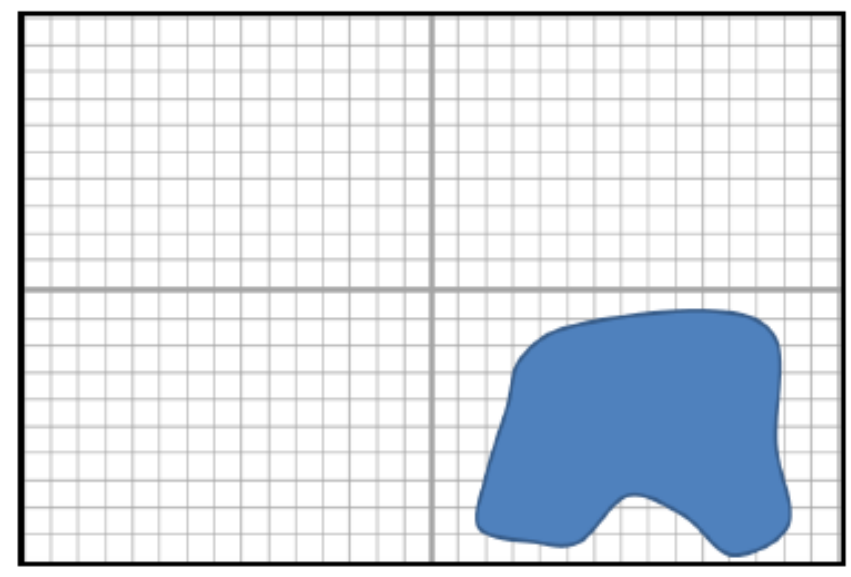

Abstract Objects Set 4 - Assessment

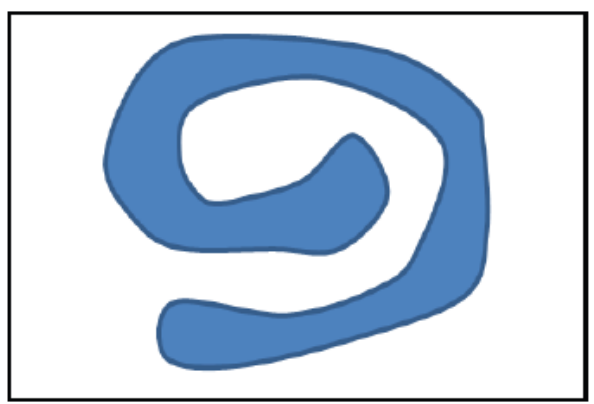

Which quadrant did the image appear in?

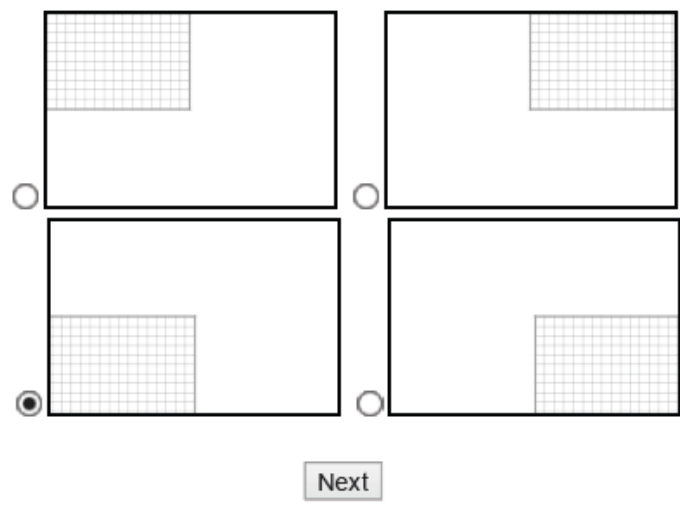

Page 82 


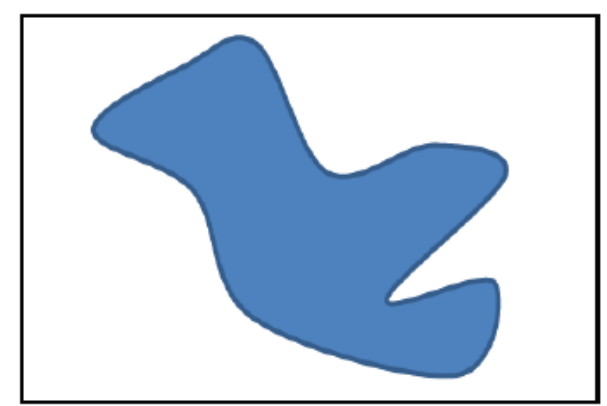

Which quadrant did the image appear in?
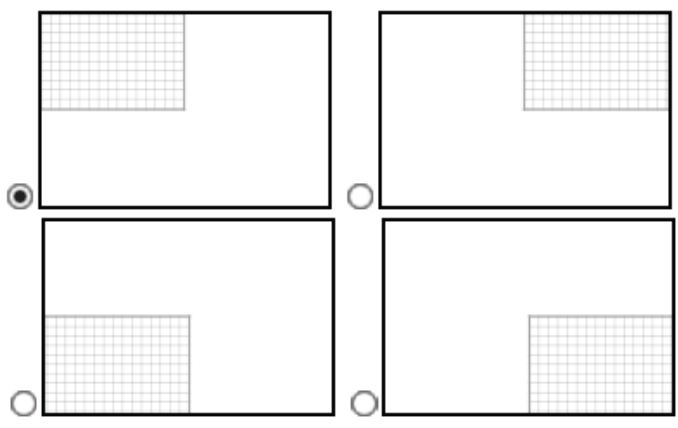

Next

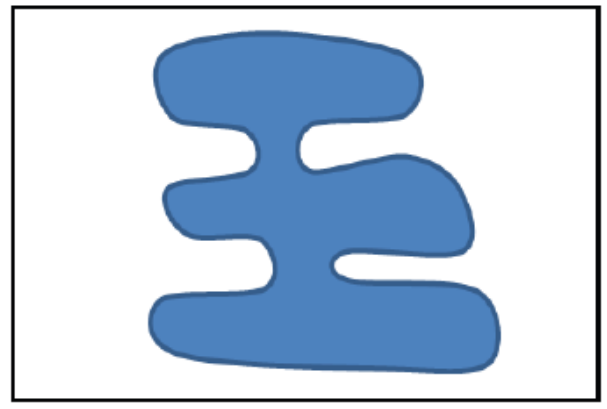

Which quadrant did the image appear in?
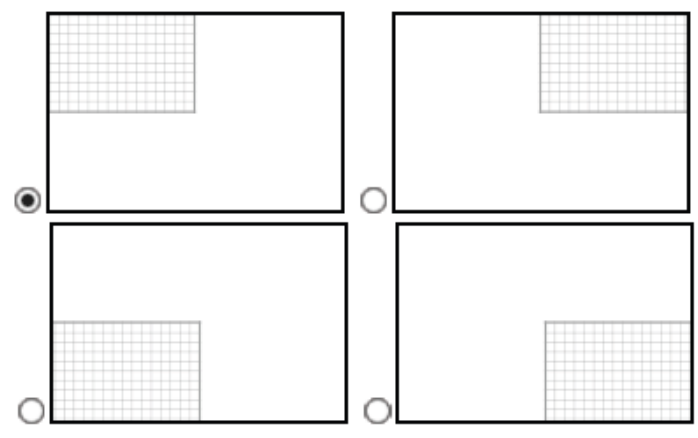

Next

Page 83 


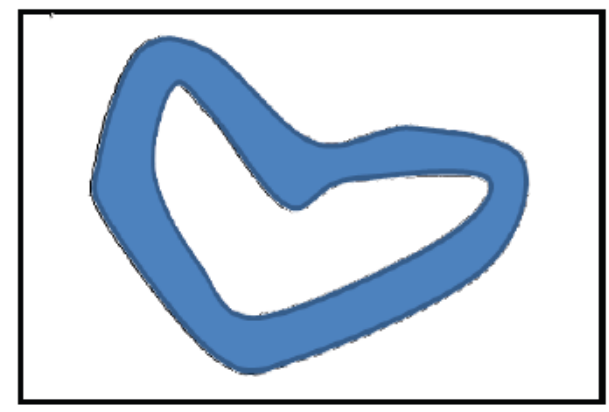

Which quadrant did the image appear in?
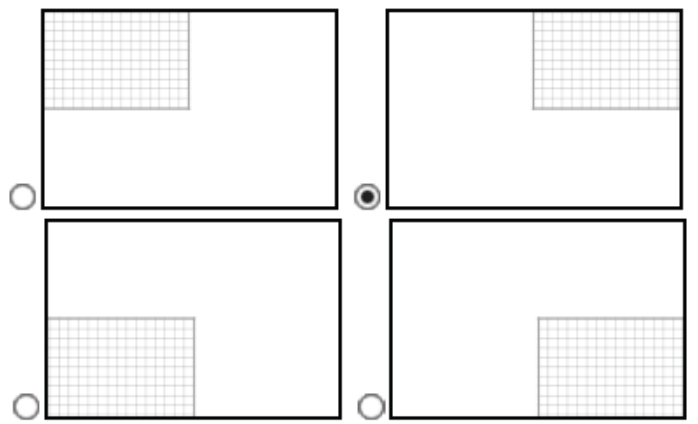

Next

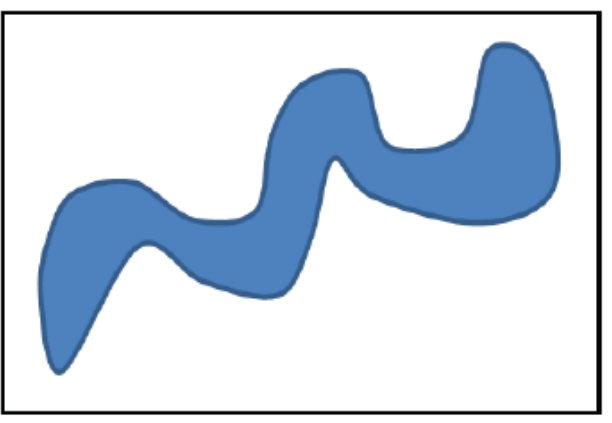

Which quadrant did the image appear in?
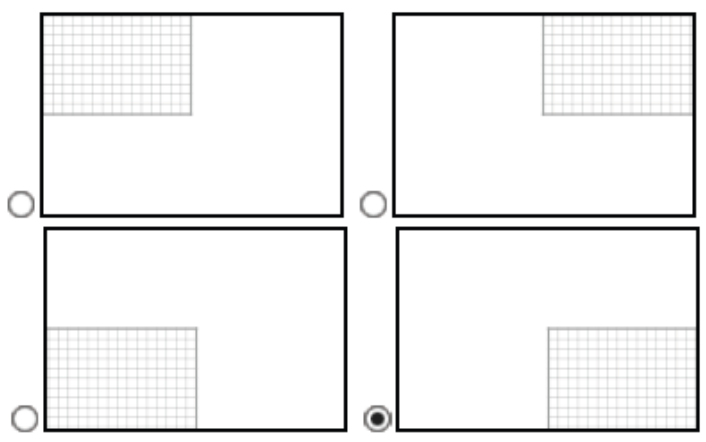

Next

Page 84 


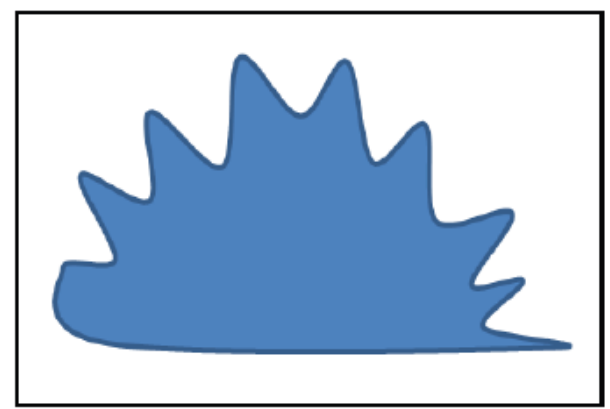

Which quadrant did the image appear in?

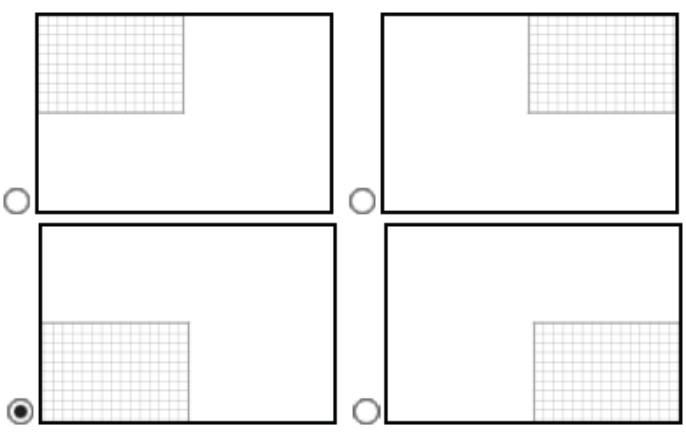

Next

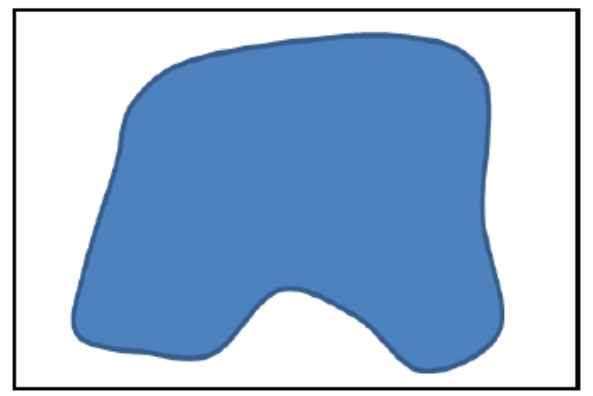

Which quadrant did the image appear in?
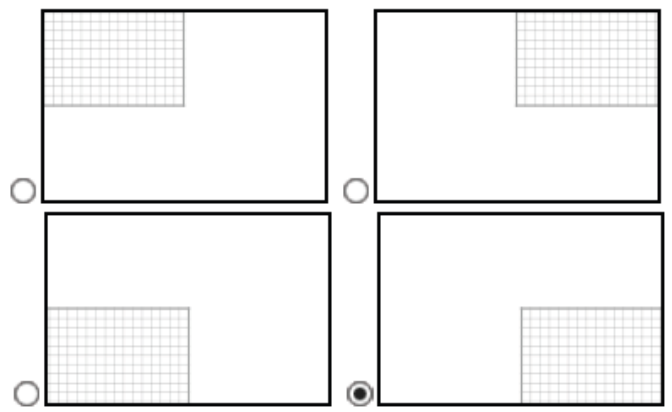

Next

Page 85 
Flags Set 1- Images
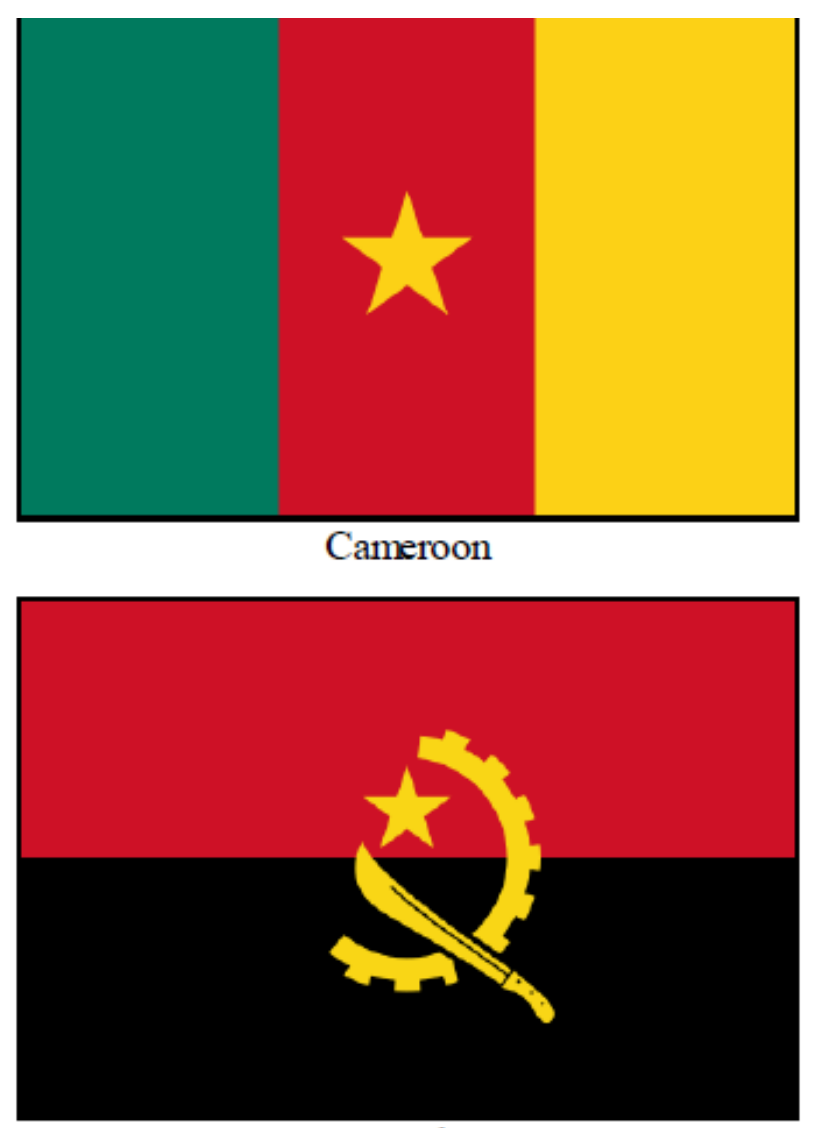

Angola

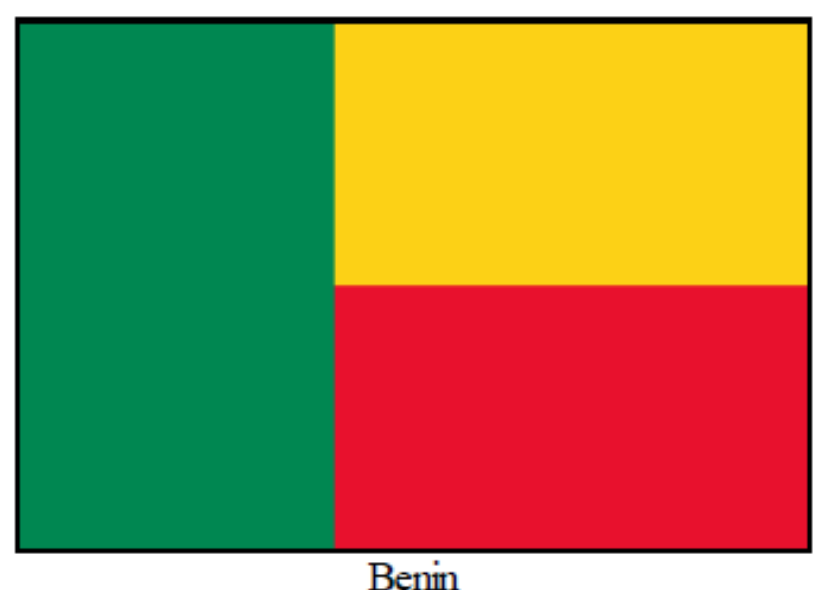

Page 86 


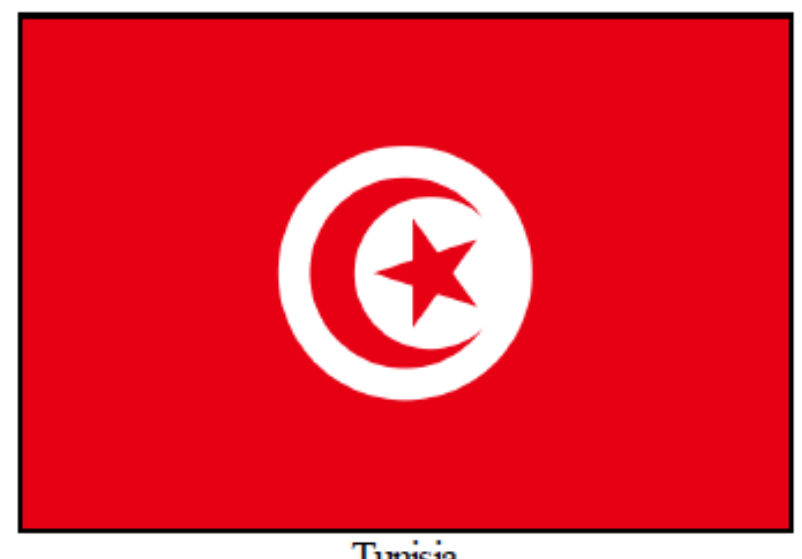

Tunisia

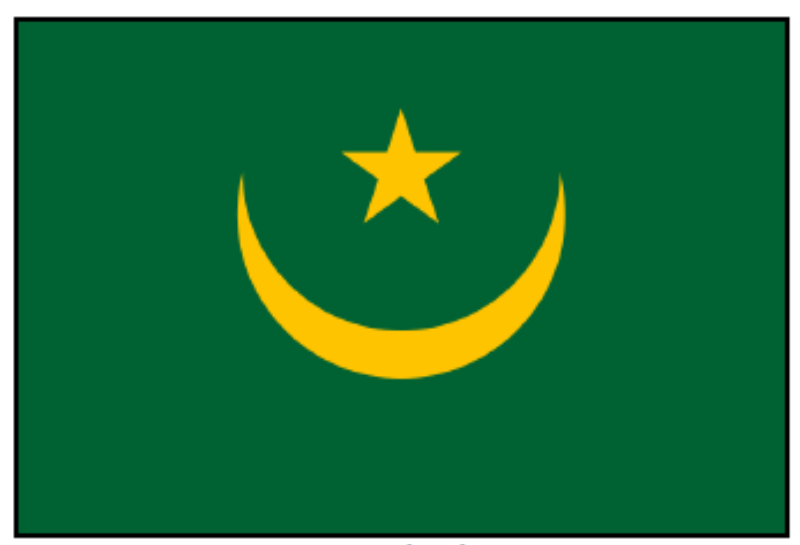

Mauritania

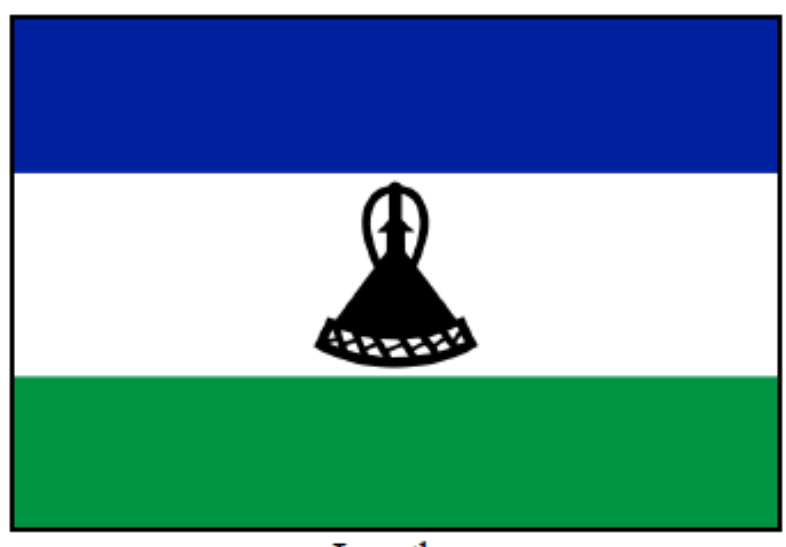

Lesotho

Page 87 


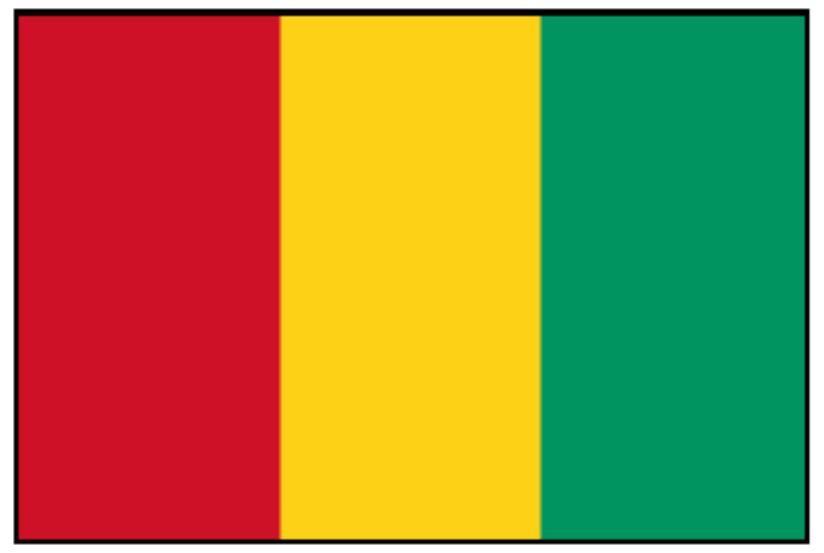

Guinea

\section{Flags Set 1- Assessment}

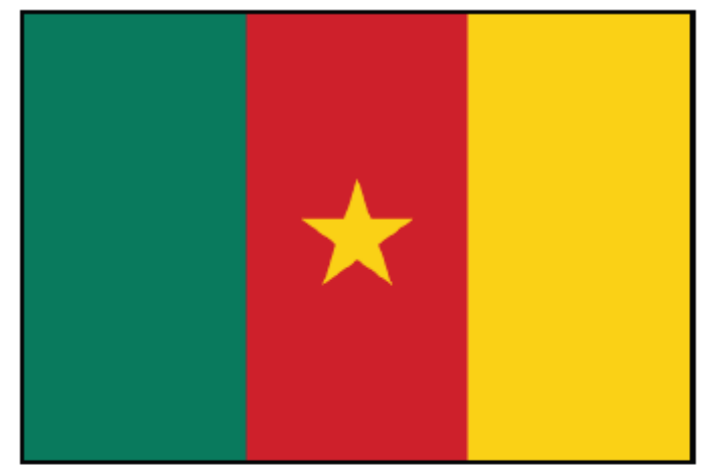

Which country does the flag above belong to?

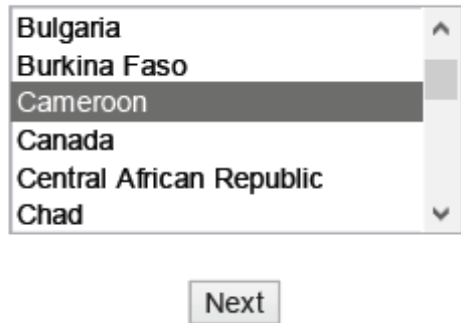

Page 88 
Which one of the following color schemes is displayed on the Angola flag?

\author{
OBhe, Yellow \\ ORed, White \\ ORed, Green, Yellow \\ O Red, Black, Yellow
}

Next

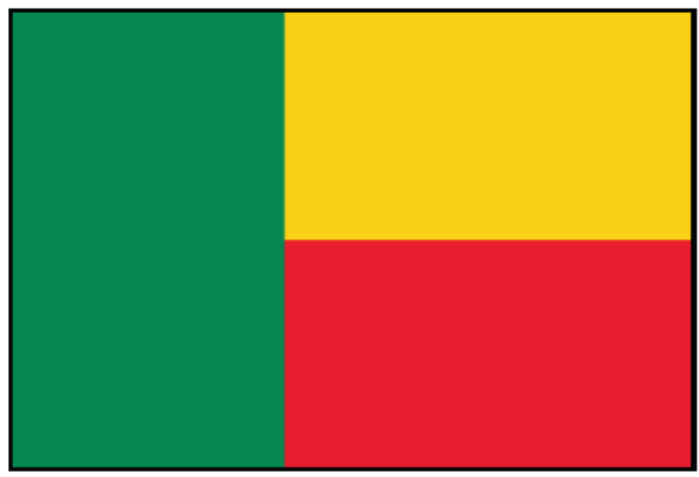

Which country does the flag above belong to?

\begin{tabular}{|l|}
\hline Belgium \\
Belize \\
\hline Benin \\
\hline Bosnia and Herzegovina \\
Bhutan \\
Bolivia \\
$\qquad$ \\
\end{tabular}

Which one of the following objects or shapes is displayed on the Tunisia flag?
Coat of Arms
Diagonal Stripes
O. Moon and Star
OHorizontal Stripes

Next

Page 89 


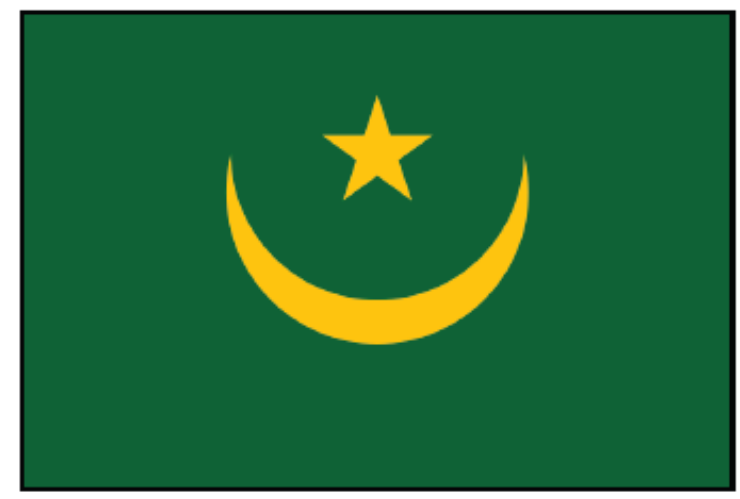

Which country does the flag above belong to?

\begin{tabular}{|l|}
\hline Mali \\
Malta \\
\hline Mauritania \\
\hline Mexico \\
Moldova \\
Monaco \\
\hline
\end{tabular}

Next

Which one of the following color schemes is displayed on the Lesotho flag?

Green, Yellow

OBhe, Green, Yellow

ORed, Green, White

OBhe, Green, White, Black

Next

Which one of the following objects or shapes is displayed on the Guinea flag?

OBird

O Vertical Stripes

OMoon and Star

OHorizontal Stripes

Next

Page 90 
Flags Set 2- Images

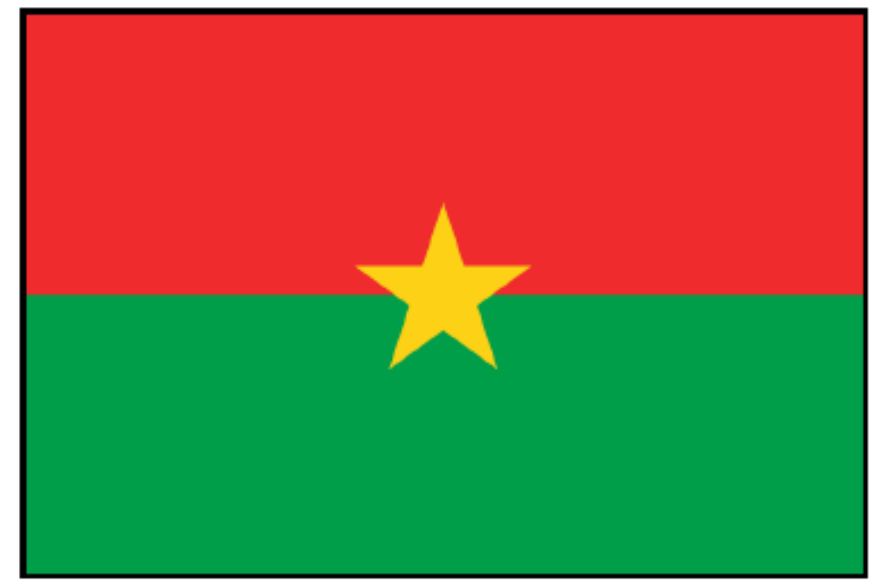

Burkina Faso

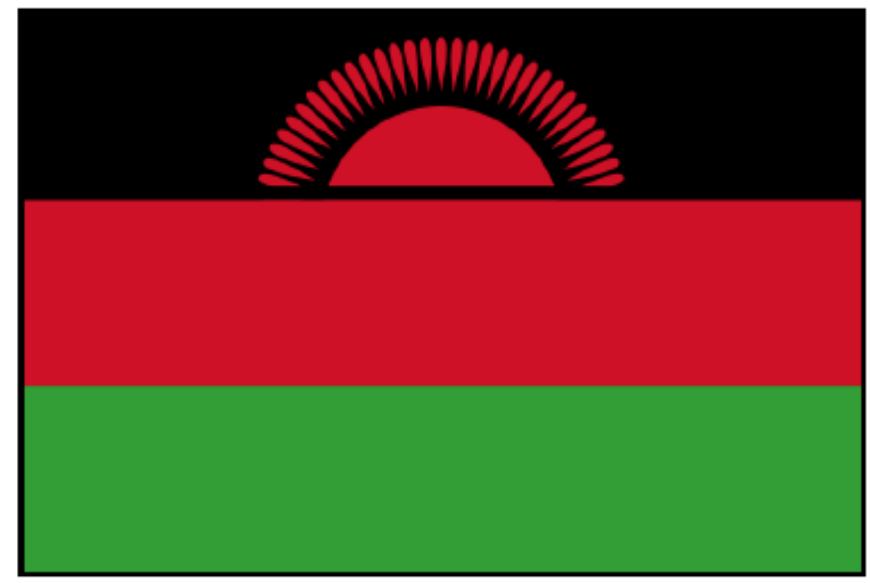

Malawi

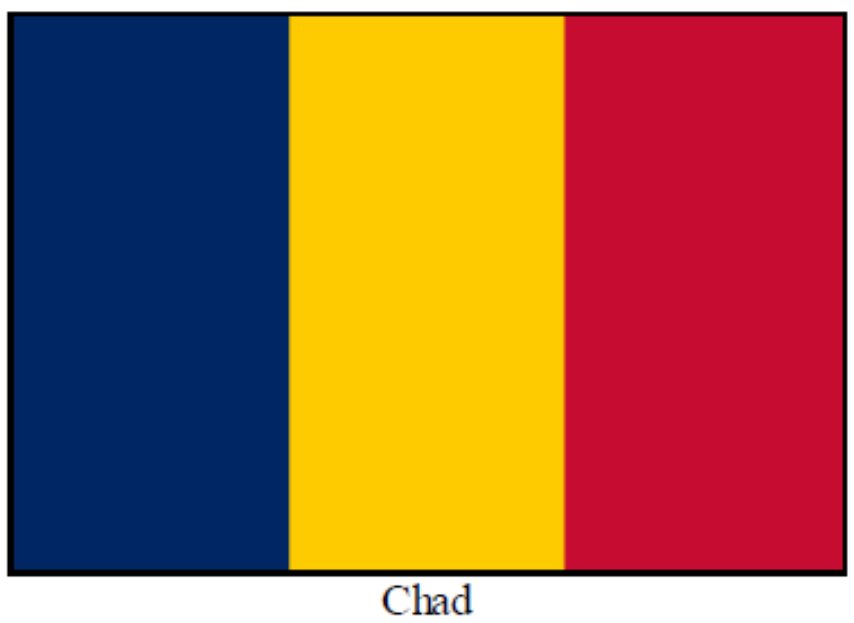

Page 91 


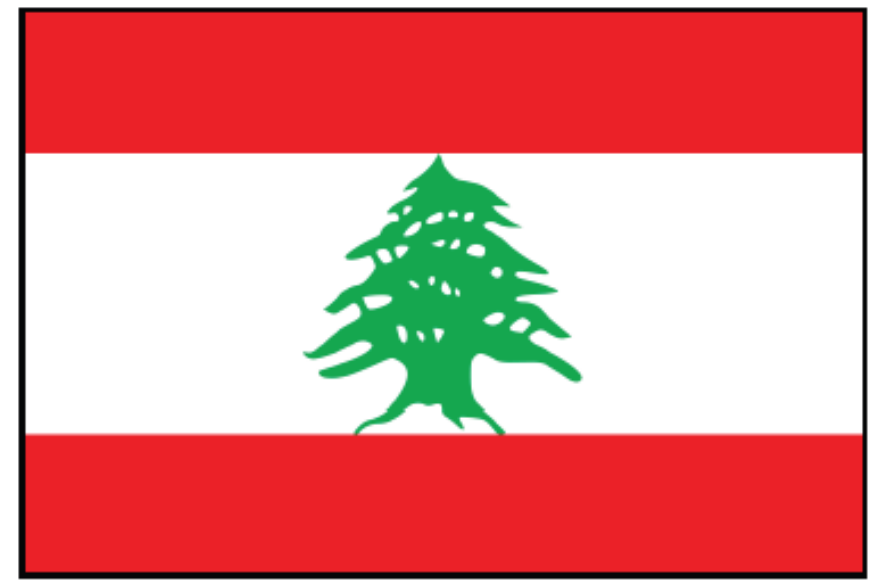

Lebanon

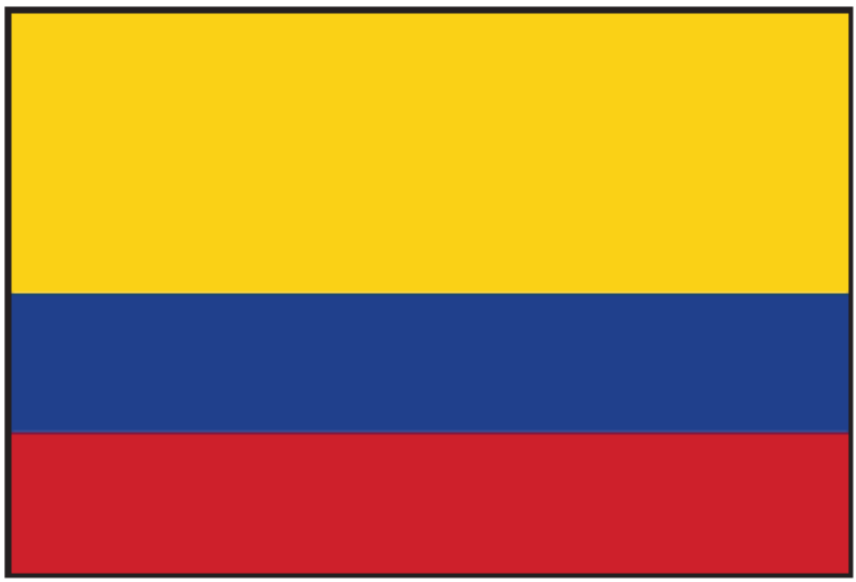

Colombia

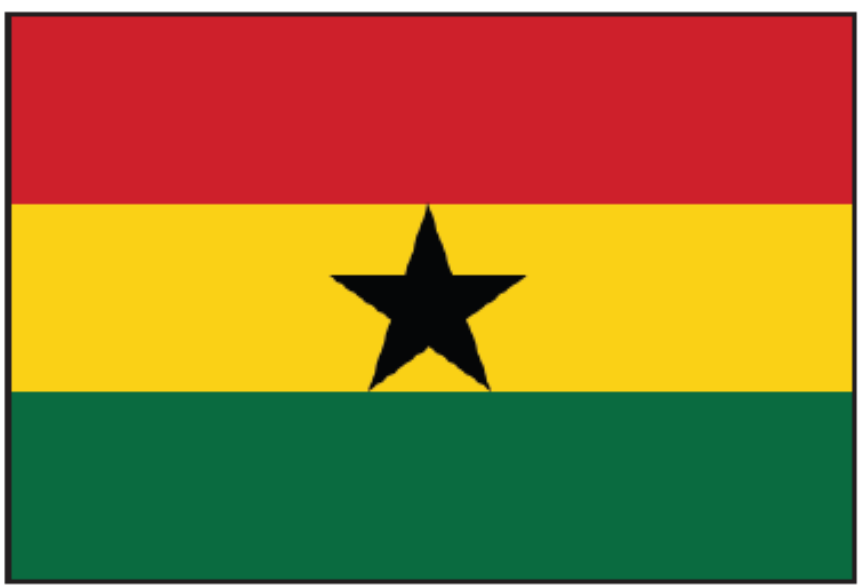

Ghana

Page 92 
Which one of the following color schemes is displayed on the Malawi flag?
O Red, Black, Green
ORed, Green, Yellow
ORed, Green, White
ORed, White

Next

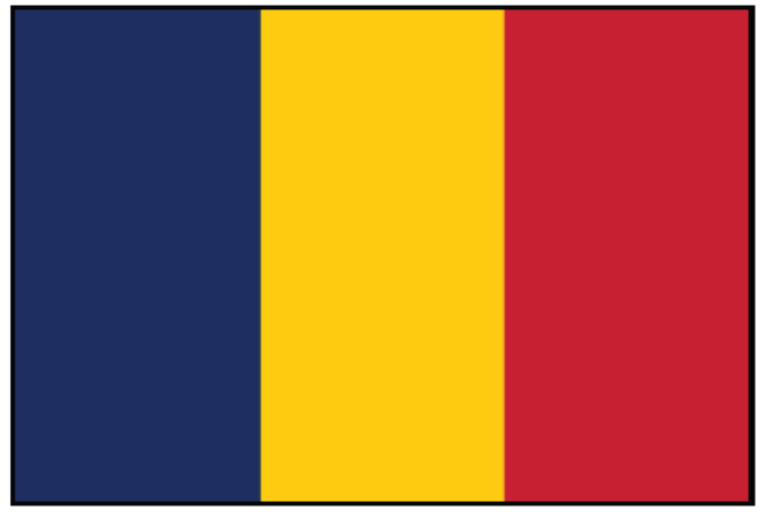

Which country does the flag above belong to?

\begin{tabular}{|l|}
\hline Canada \\
Central African Republic \\
\hline Chad \\
\hline Chile \\
China \\
Colombia \\
\hline
\end{tabular}

Next

Which one of the following objects or shapes is displayed on the Lebanon flag?

OSun

Vertical Stripes

OStar

O. Tree

Next

Page 94 


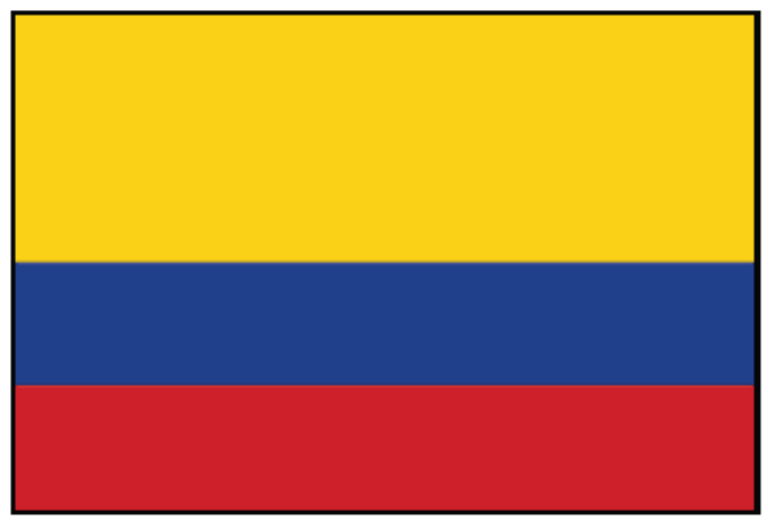

Which country does the flag above belong to?

\begin{tabular}{|ll|}
\hline Chile & \\
China & \\
\hline Colombia & \\
\hline Congo & \\
Costa Rica & \\
Croatia & \\
\hline & Next \\
&
\end{tabular}

Which one of the following color schemes is displayed on the Ghana flag?
OBhe, Green, Yellow
Green, Yellow
O Red, Green, Yellow, Black
ORed, Green, White

Next

Which one of the following objects or shapes is displayed on the Zambia flag?
OHorizontal Stripes
OSun
OStar
OBird

Next 
Flags Set 3- Images

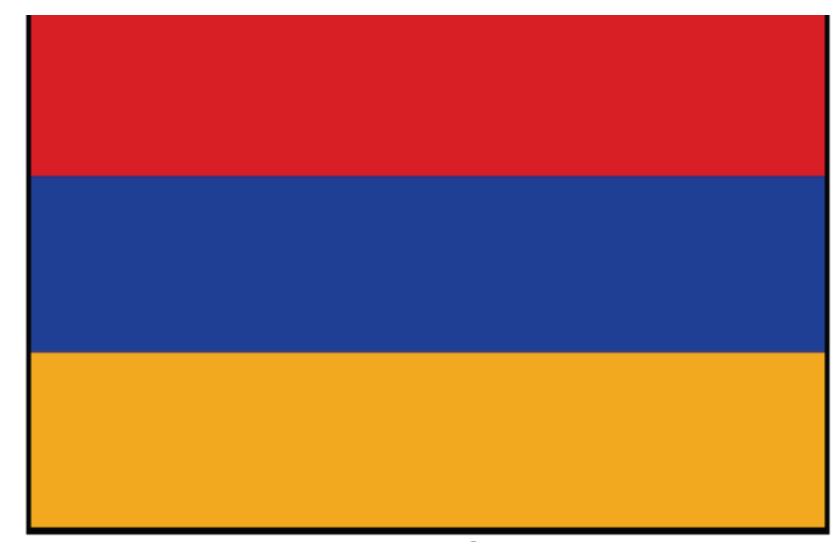

Armenia

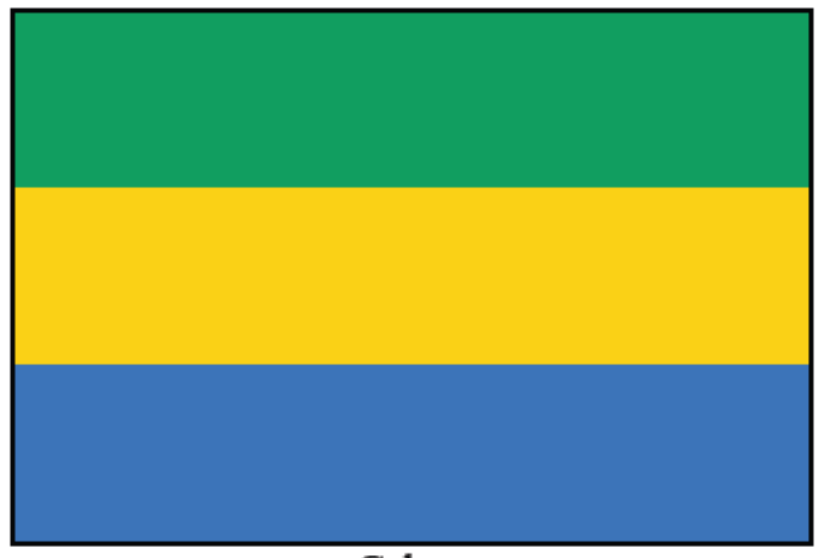

Gabon

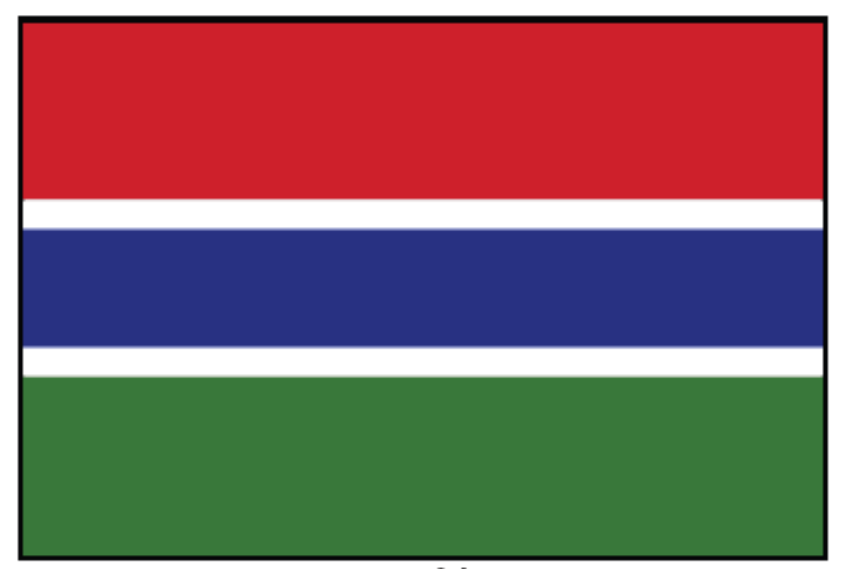

Gambia

Page 96 

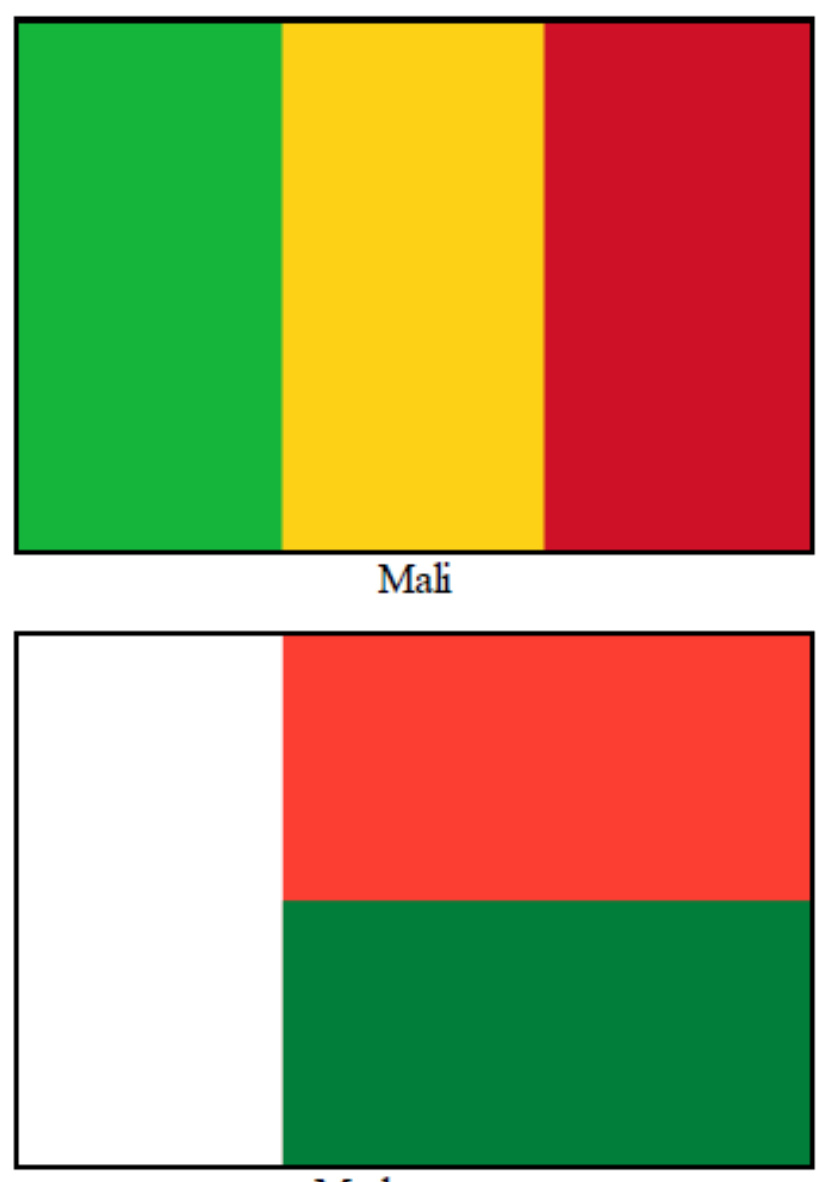

Madagascar

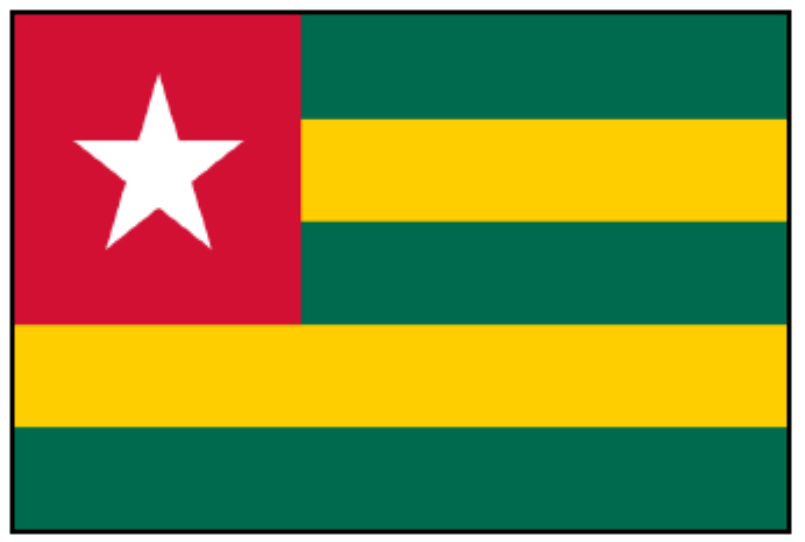

Togo

Page 97 


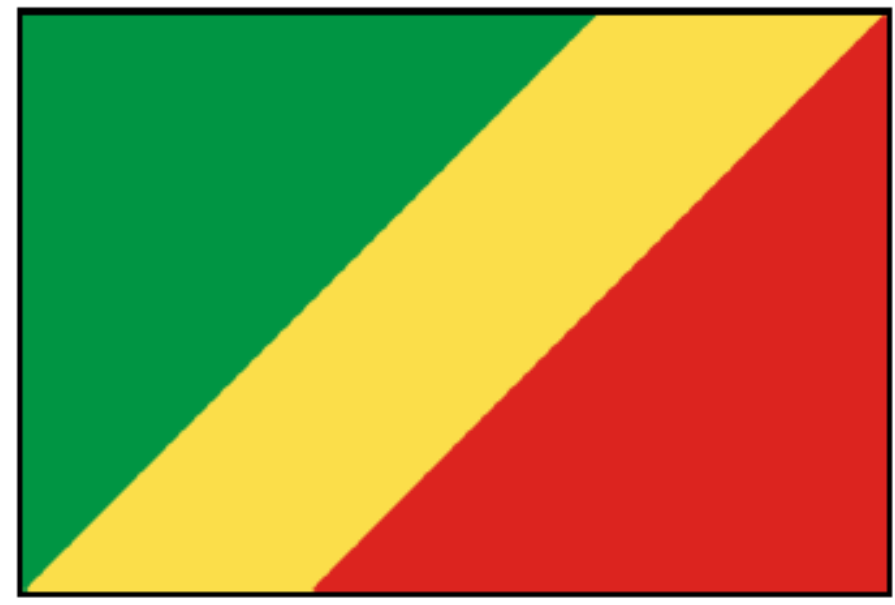

Congo

Flags Set 3- Assessment

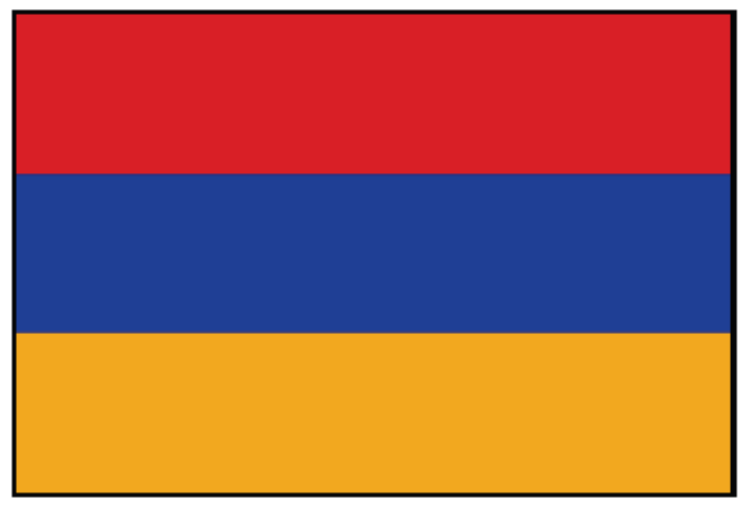

Which country does the flag above belong to?

\begin{tabular}{|l|}
\hline Antigua and Barbuda \\
Argentina \\
\hline Armenia \\
\hline Austrailia \\
Azerbaijan \\
Bahamas \\
\hline
\end{tabular}

Next 
Which one of the following color schemes is displayed on the Gabon flag?

ORed, Green, Yellow

ORed, Bhe, Yellow

Oreen, Yellow

OBhe, Green, Yellow

Next

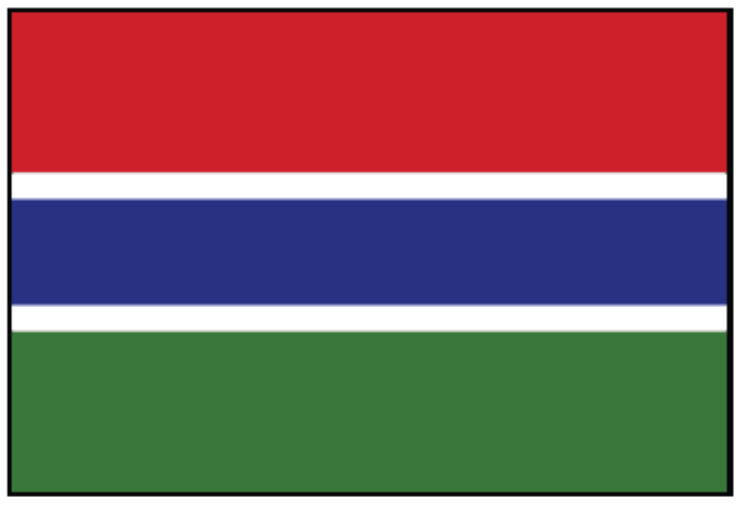

Which country does the flag above belong to?

\begin{tabular}{|l|l|}
\hline $\begin{array}{l}\text { French Guiana } \\
\text { Gabon }\end{array}$ & $\wedge$ \\
\hline Gambia & \\
\hline Georgia & \\
Germany & \\
Ghana & \\
& Next \\
\hline
\end{tabular}

Which one of the following objects or shapes is displayed on the Mali flag?

O Vertical Stripes
Sun
ODiagonal Stripes
Star




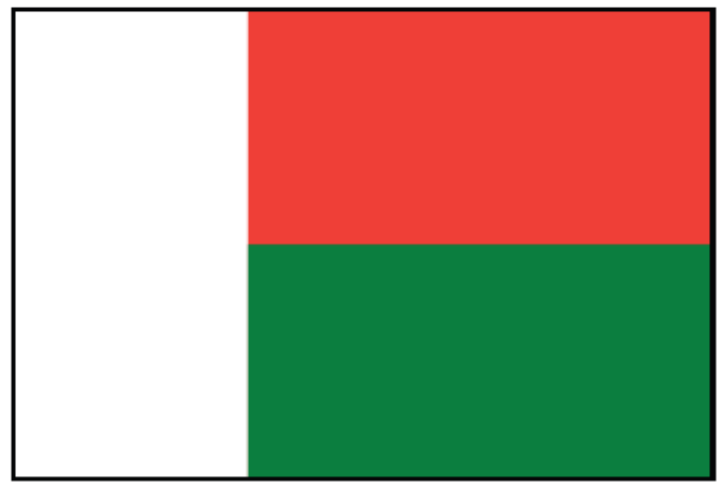

Which country does the flag above belong to?

\begin{tabular}{|ll|}
\hline $\begin{array}{ll}\text { Luxembourg } \\
\text { Macedonia }\end{array}$ \\
\hline Madagascar & \\
\hline Malaysia & \\
Malawi & \\
Mali & \\
\hline & Next \\
\hline
\end{tabular}

Which one of the following color schemes is displayed on the Togo flag?
Green, Yellow
OBhe, Green, Yellow
Red, Green, White
OBhe, Green, White, Black

\section{Next}

Which one of the following objects or shapes is displayed on the Congo flag?
Coat of Arms
O Diagonal Stripes
OMoon and Star
OHorizontal Stripes 
Flags Set 4- Images

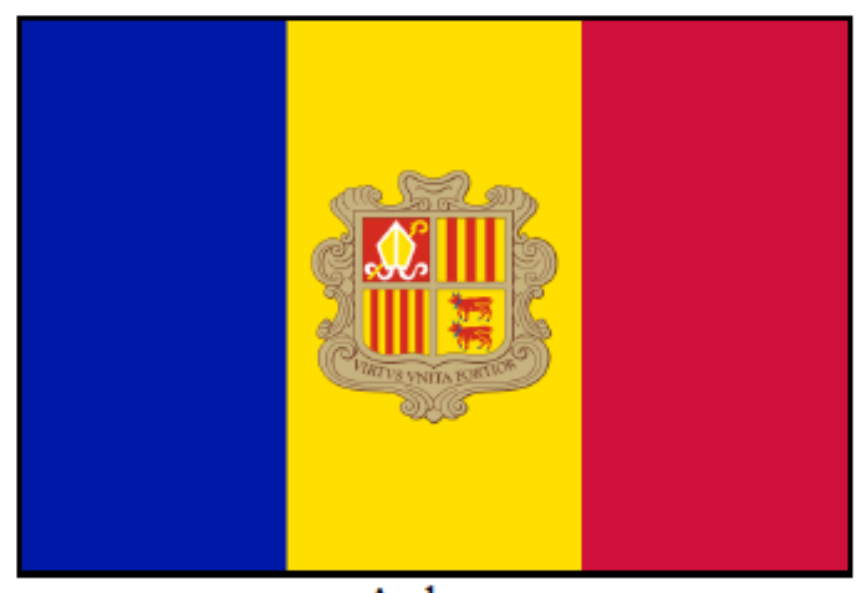

Andorra

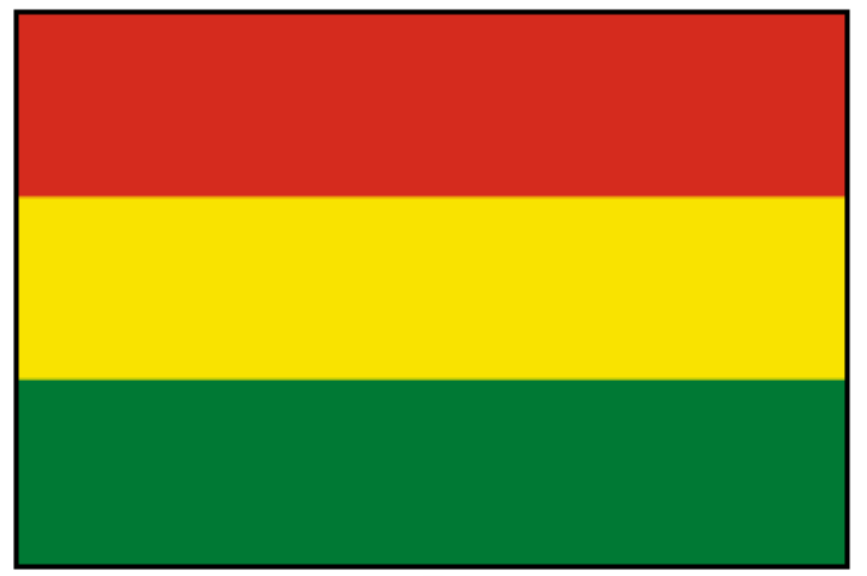

Bolivia

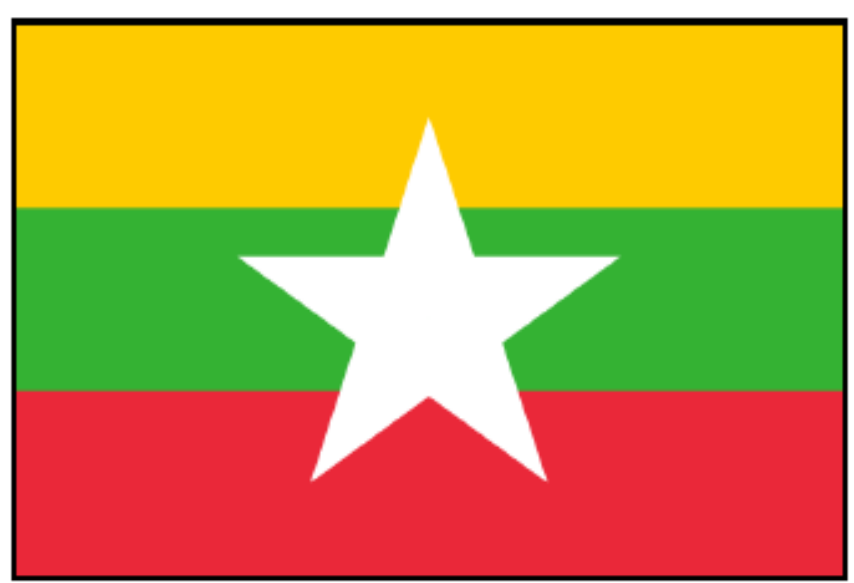

Myanmar

Page 101 


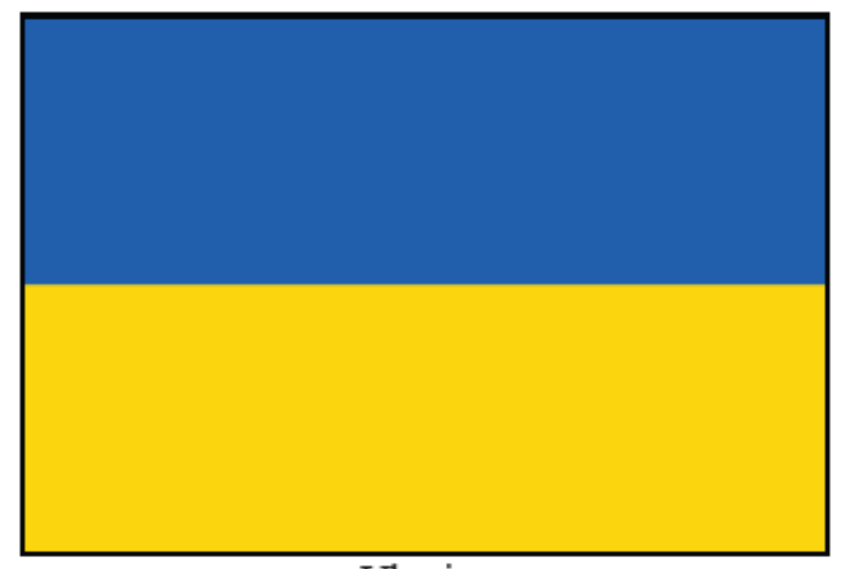

Ukraine
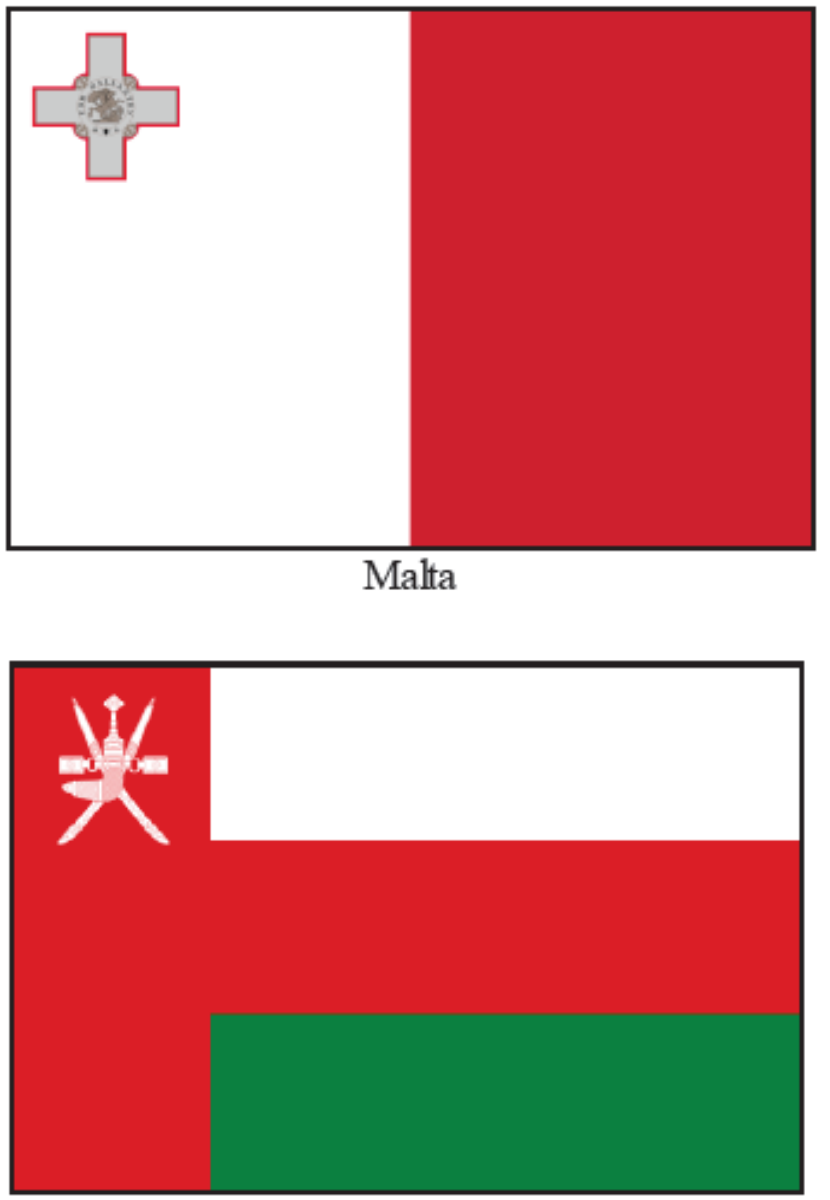

Oman

Page 102 


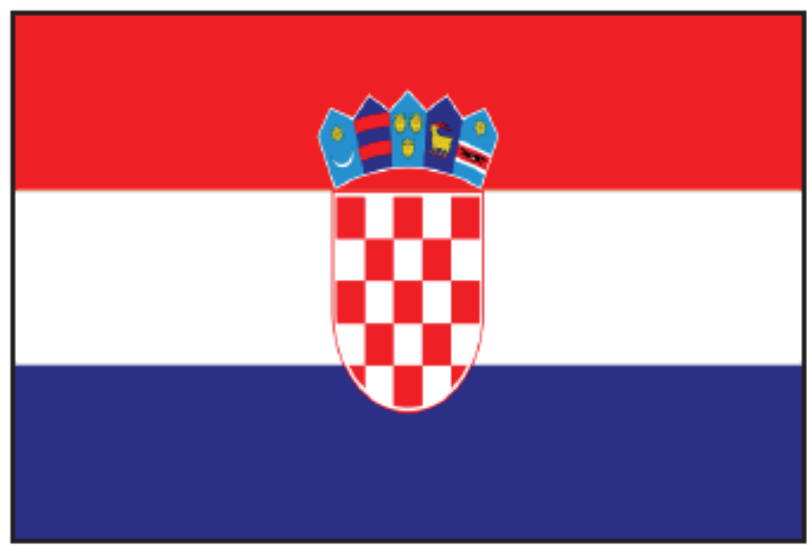

Croatia

Flags Set 4- Assessment

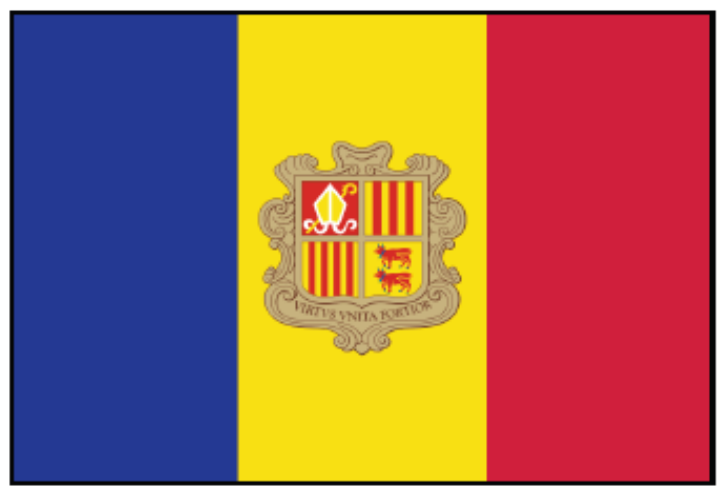

Which country does the flag above belong to?

\begin{tabular}{|c|c|}
\hline \multirow[t]{2}{*}{$\begin{array}{l}\text { Albania } \\
\text { Algeria } \\
\text { Andorra }\end{array}$} & $\wedge$ \\
\hline & \\
\hline $\begin{array}{l}\text { Angola } \\
\text { Antigua and Barbuda } \\
\text { Argentina }\end{array}$ & \\
\hline Next & \\
\hline
\end{tabular}

Page 103 
Which one of the following color schemes is displayed on the Bolivia flag?

ORed, White

ORed, Green, Yellow

Green, Yellow

ORed, Black, Yellow

Next

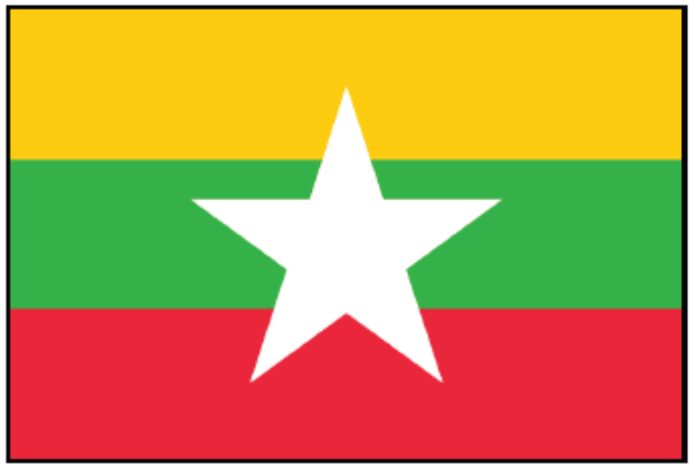

Which country does the flag above belong to?

\begin{tabular}{|l|}
\hline Morocco \\
Mozambique \\
\hline Myanmar \\
\hline Namibia \\
Nepal \\
Netherlands \\
\hline
\end{tabular}

Next

Which one of the following objects or shapes is displayed on the Ukraine flag?

Overtical Stripes

OBird

Ostar

O Horizontal Stripes

Next 


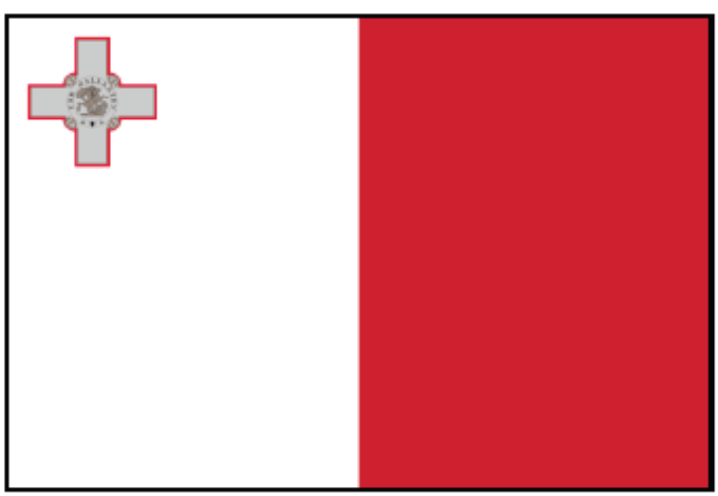

Which country does the flag above belong to?

\begin{tabular}{|ll|}
\hline Malawi & \\
\hline Mali & \\
\hline Malta & \\
\hline Mauritania & \\
\hline Mexico & \\
\hline Moldova & \\
\hline & Next \\
\hline
\end{tabular}

Which one of the following color schemes is displayed on the Oman flag?
Green, Yellow
Red, Black, Yellow
Red, White
(2) Red, Green, White

Next

Which one of the following objects or shapes is displayed on the Croatia flag?
(-) Coat of Arms
Moon and Star
Sun
Vertical Stripes

Next 


\section{Art Set 1 - Images}

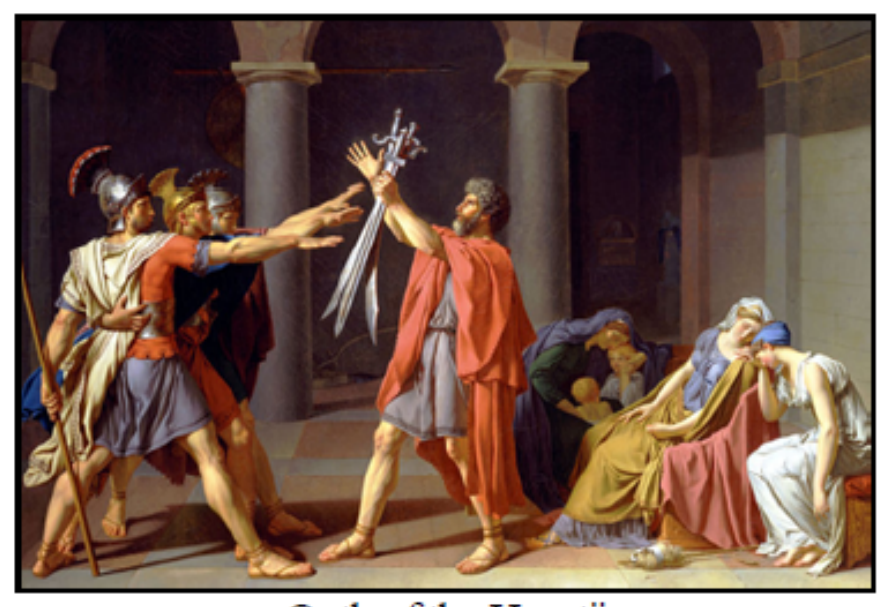

Oath of the Horatii

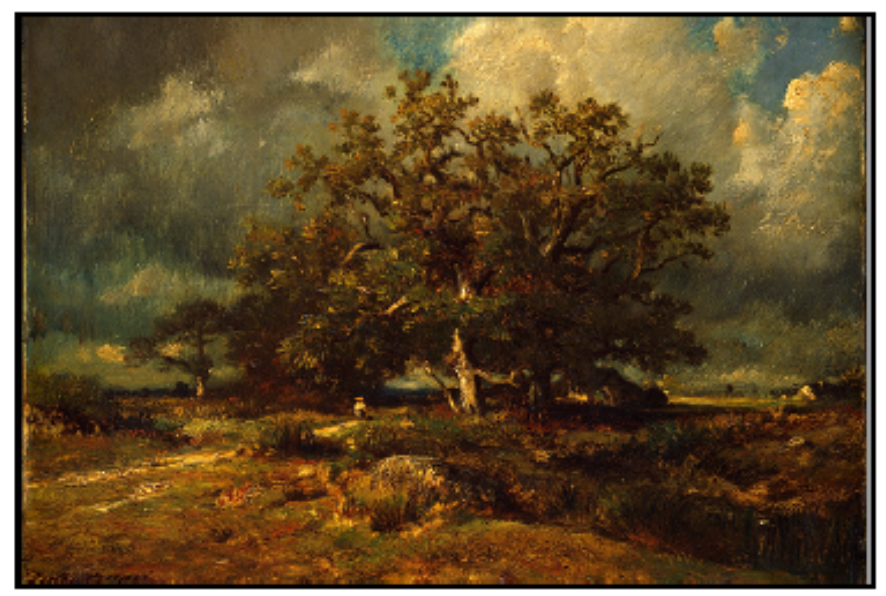

The Old Oak

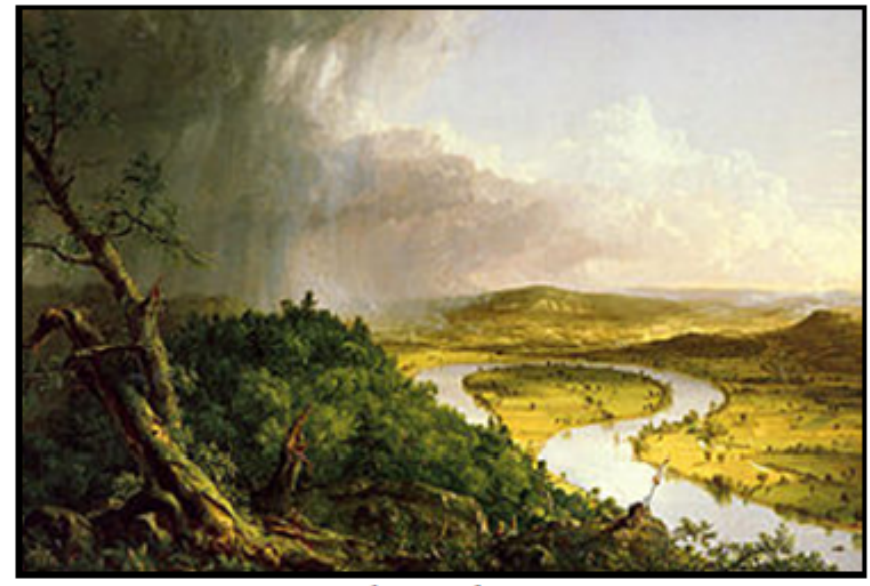

The Oxbox

Page 106 


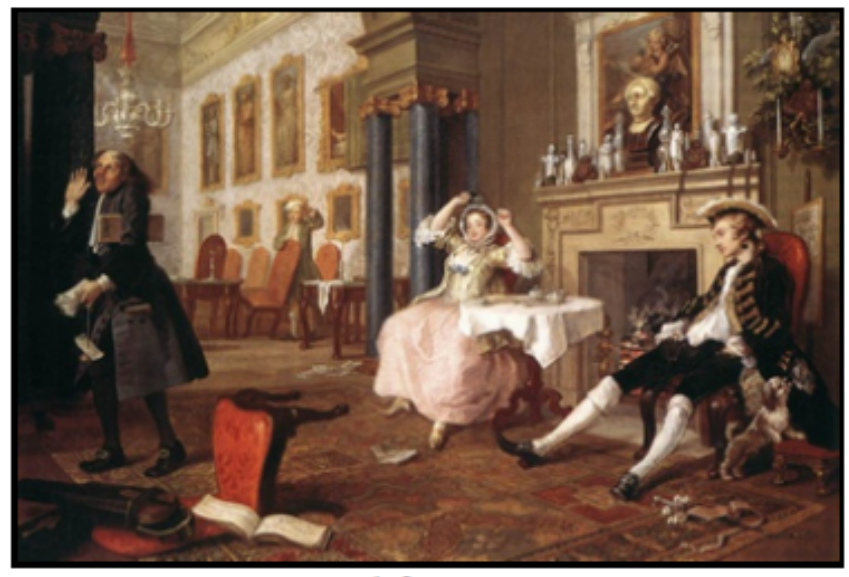

Breakfast Scene

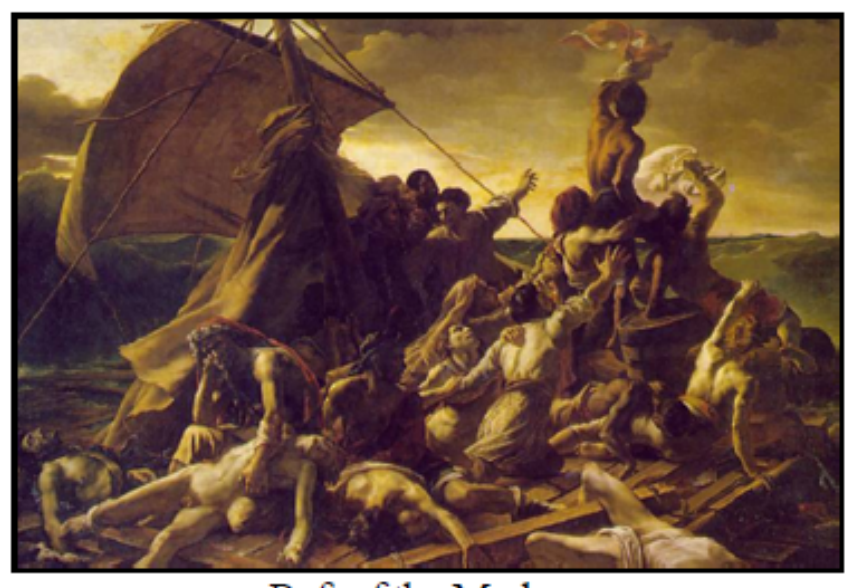

Raft of the Medusa

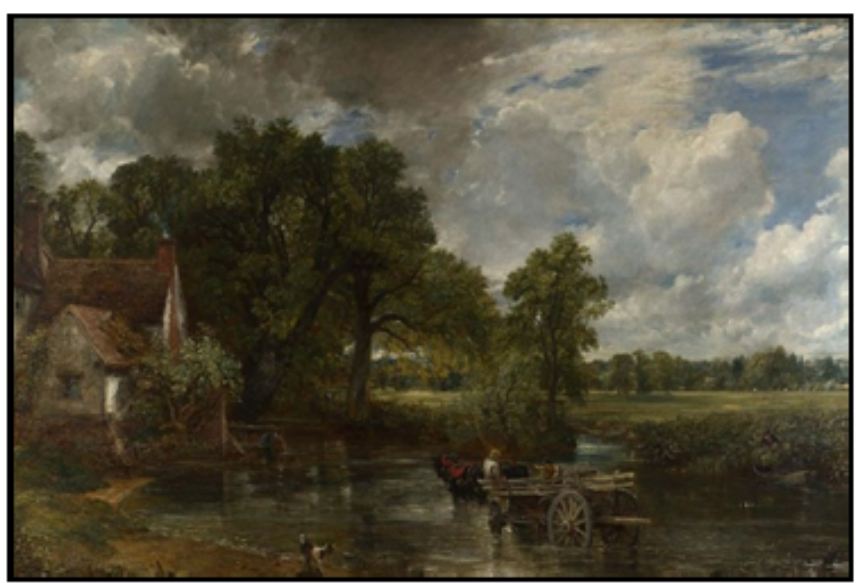

The Haywain

Page 107 


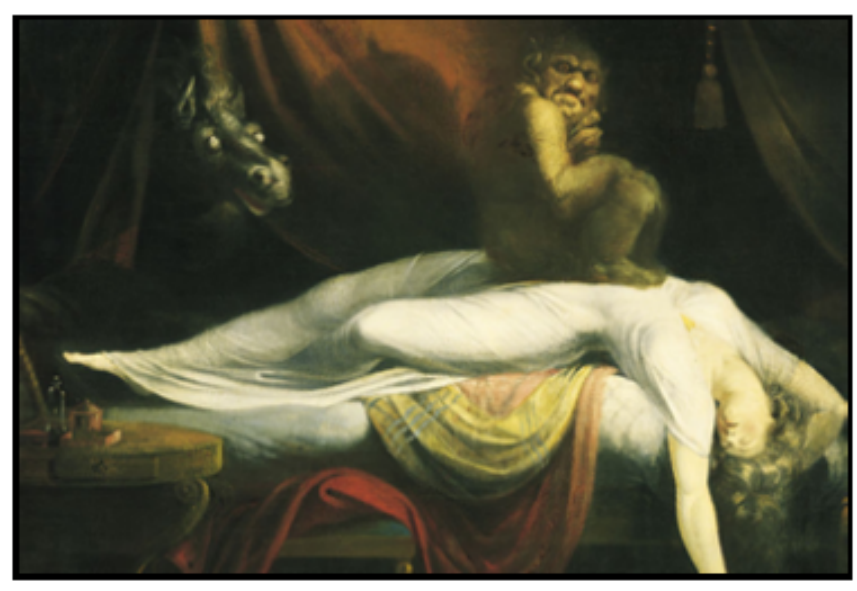

The Nightmare

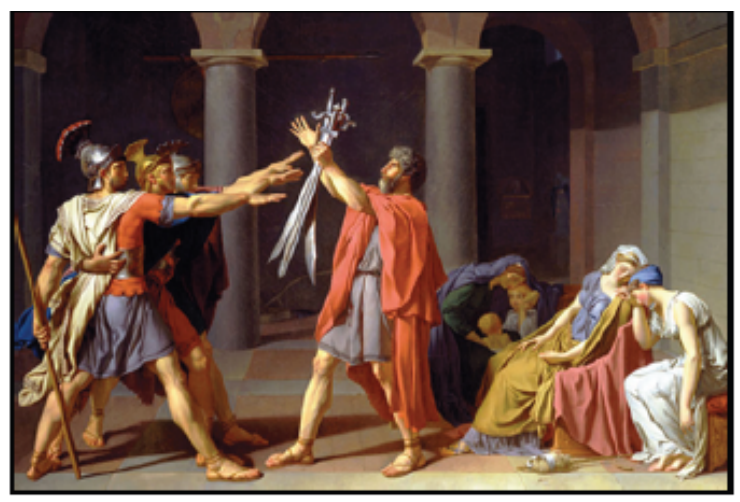

What is the name of the painting above?

\begin{tabular}{|l|}
\hline Breakfast Scene \\
The Haywain \\
The Nightmare \\
\hline Oath of the Horatii \\
\hline The Old Oak \\
The Oxbow \\
Raft of the Medusa \\
\hline
\end{tabular}

Next

Page 108 
What is the setting of the painting The Old Oak?

Outside in a Field or Grassy Area

Onside

Outside on a Battle Field

Outside on the Water

Next

What is the primary subject of the painting The Oxbow?

\author{
OLandscape and Animals \\ O People and Horses \\ OPeople \\ OLandscape
}

Next

How many people are in the painting Breakfast Scene?

ONone

OMore than 10

O At least 1 and at most 5

$O$ At least 6 and at most 10

Next

Page 109 
What is the setting of the painting Raft of the Medusa?

Outside in a Field or Grassy Area

OInside

Outside on a Battle Field

O Outside on the Water

Next

What is the primary subject of the painting The Haywain?
People and Horses
OLandscape
OPeople
Landscape and Animals

Next

How many people are in the painting The Nightmare?
(-) At least 1 and at most 5
At least 6 and at most 10
ONone
More than 10

Next 


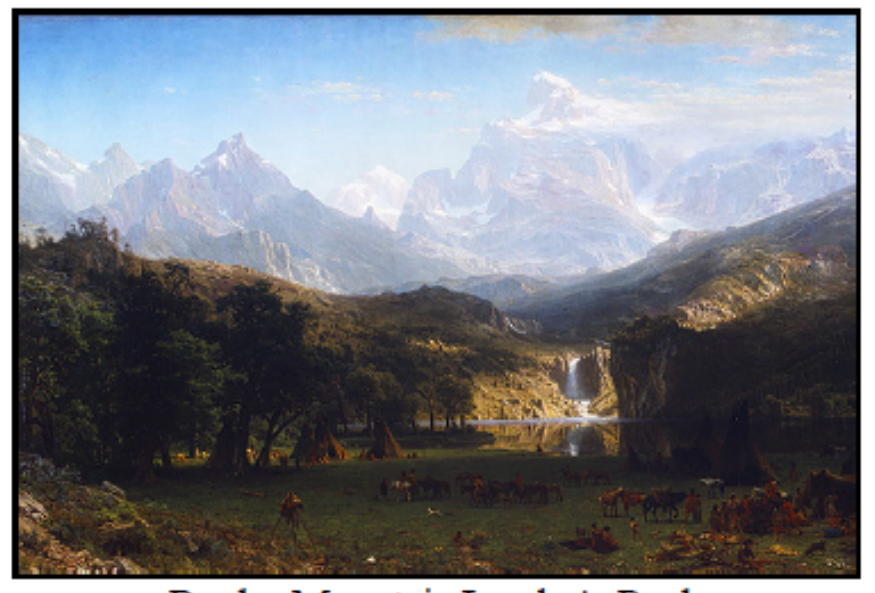

Rocky Mountain Lander's Peak

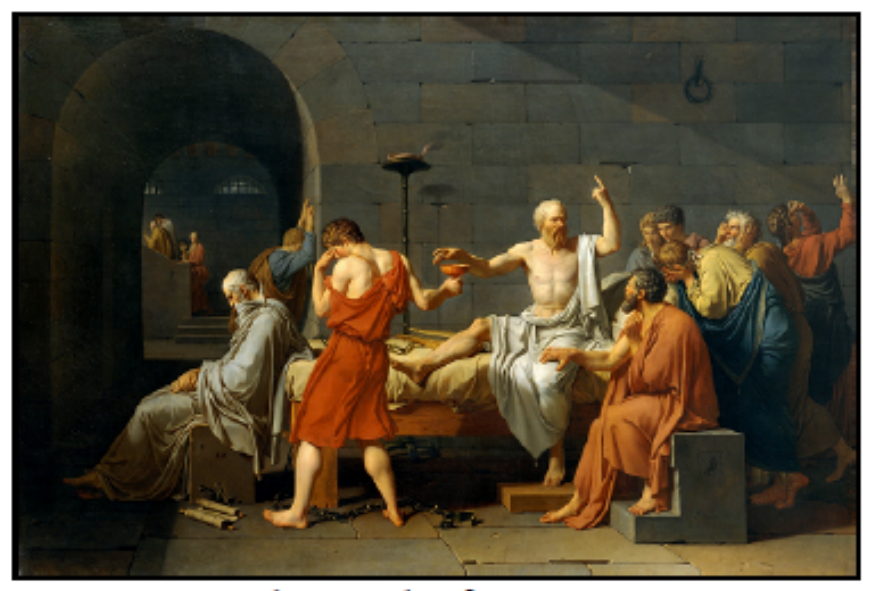

The Death of Socrates

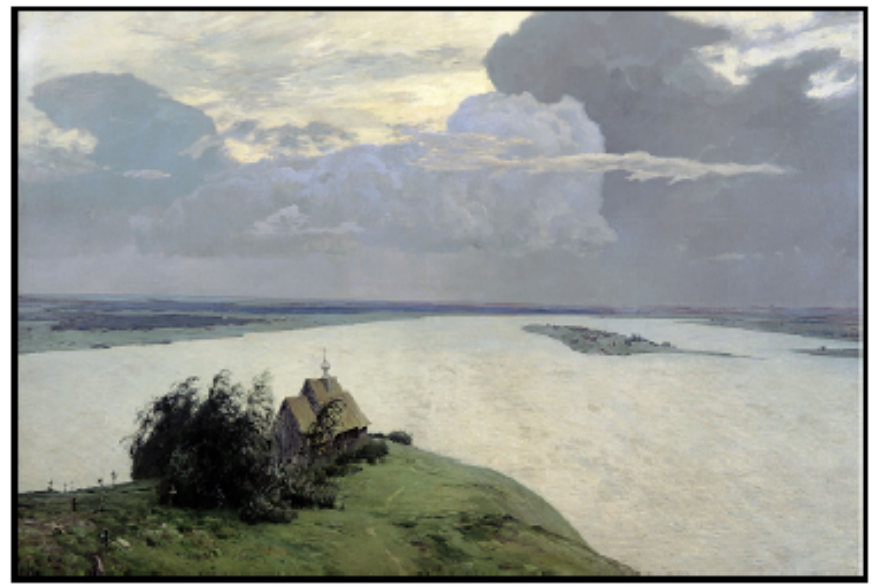

Above Eternal Peace

Page 111 


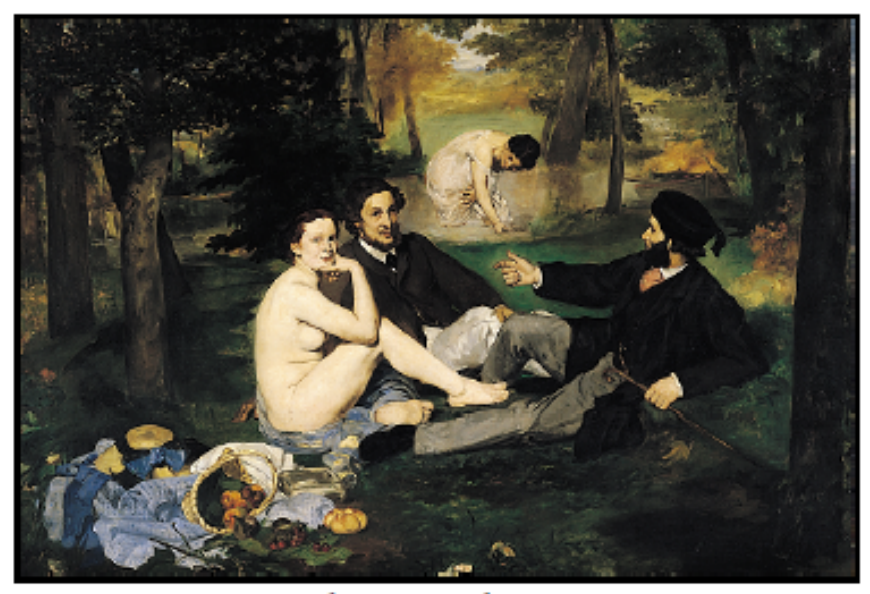

Luncheon on the Grass

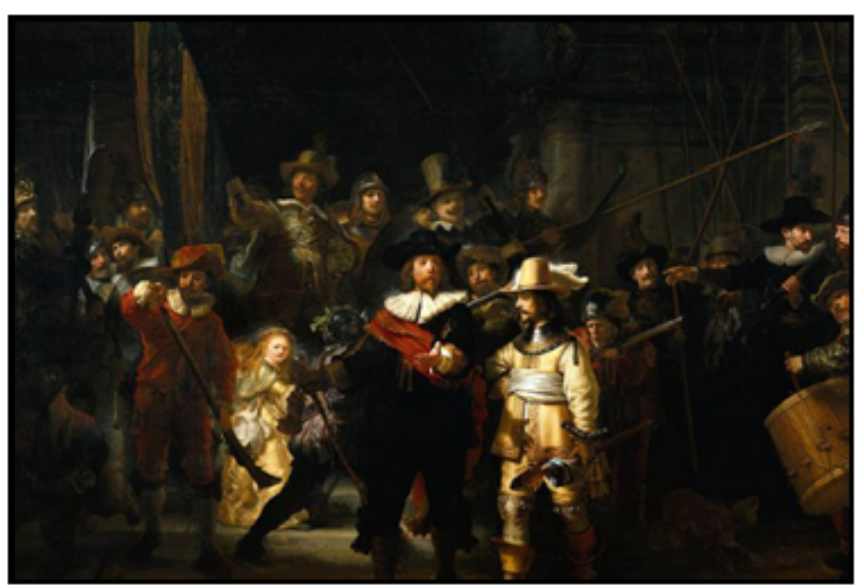

The Night Watch

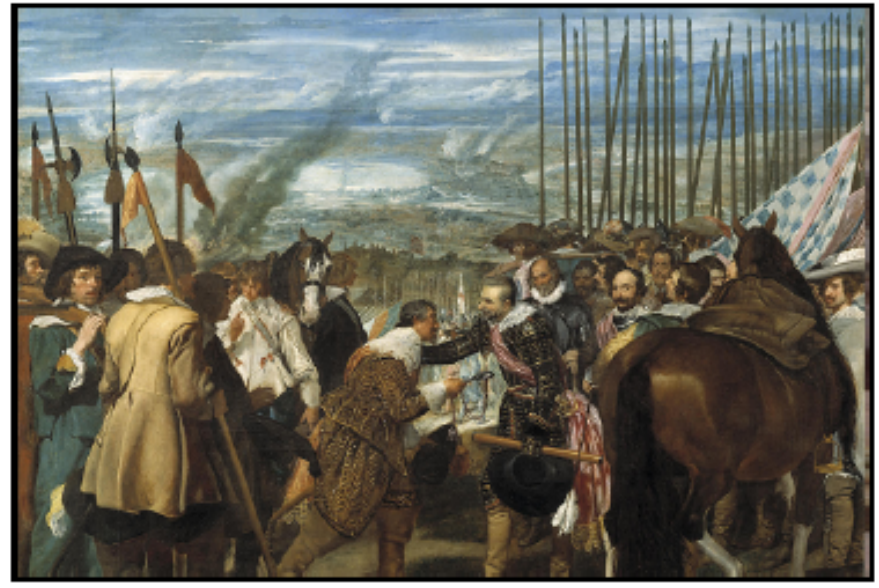

The Surrender of Breda

Page 112 


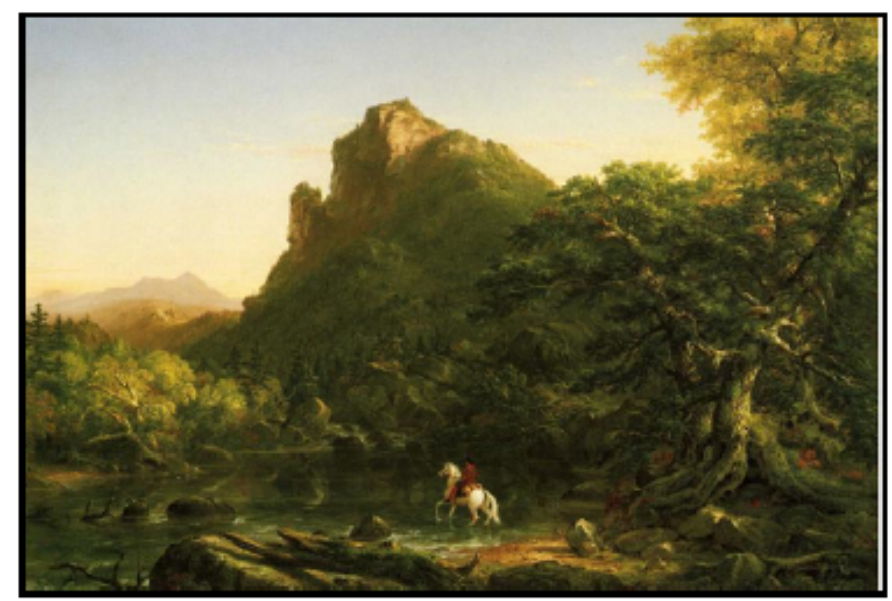

The Mountain Ford

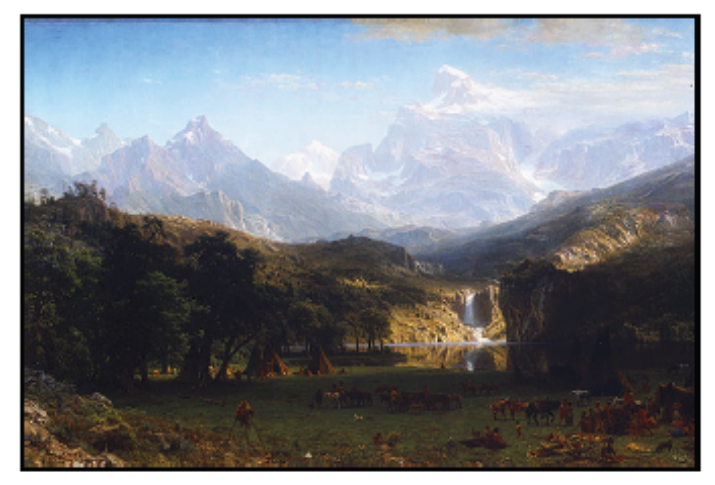

What is the name of the painting above?

\begin{tabular}{l} 
Above Etemal Peace \\
The Death of Socrates \\
Luncheon on the Grass \\
The Mountain Ford \\
The Night Watch \\
\hline Rocky Mountain Lander's Peak \\
\hline The Surrender of Breda \\
\hline
\end{tabular}

Next

Page 113 
What is the setting of the painting The Death of Socrates?

O Inside

Outside on the Water

Outside on a Battle Field

Outside in a Field or Grassy Area

Next

What is the primary subject of the painting Above Eternal Peace?

OPeople

OLandscape and Animals

O People and Horses

OLandscape

Next

How many people are in the painting Luncheon on the Grass?

- At least 1 and at most 5

ONone

At least 6 and at most 10

OMore than 10

Next 
What is the setting of the painting The Night Watch?

\author{
Outside in a Field or Grassy Area \\ Outside on the Water \\ O) Outside on a Battle Field \\ OInside
}

Next

What is the primary subject of the painting The Surrender of Breda?

\author{
OLandscape and Animals \\ - People and Horses \\ Landscape \\ People
}

Next

How many people are in the painting The Mountain Ford?

OMore than 10

O At least 1 and at most 5

ONone

At least 6 and at most 10

Next

Page 115 


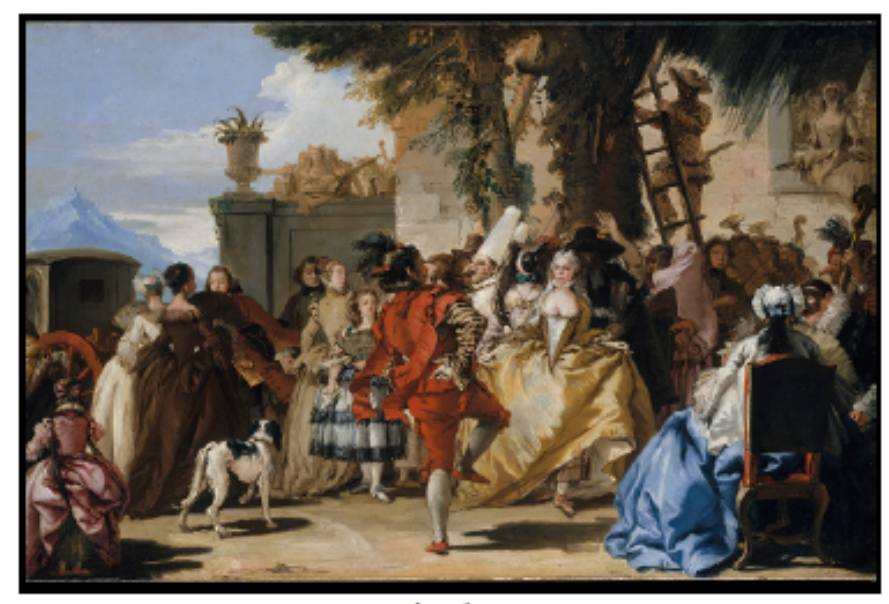

A Dance in the Country

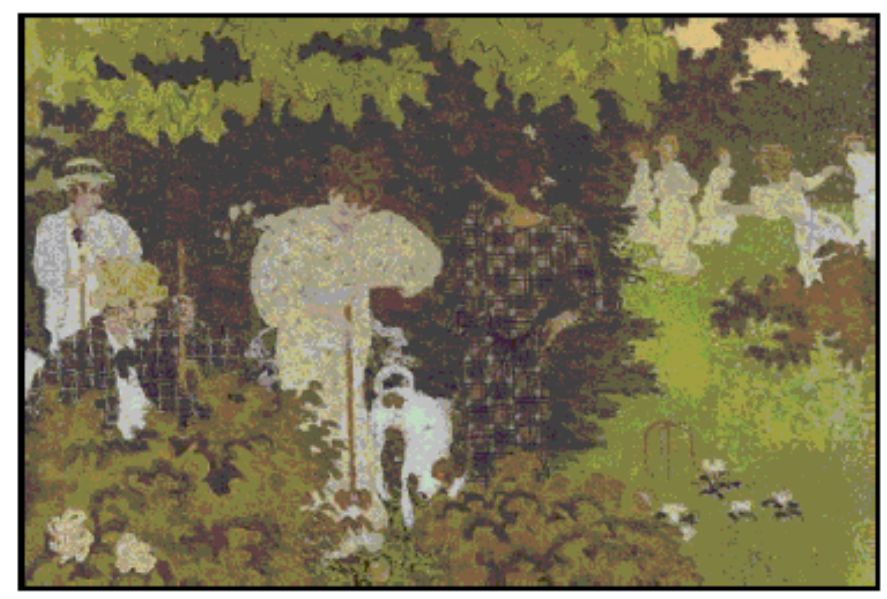

Twilight

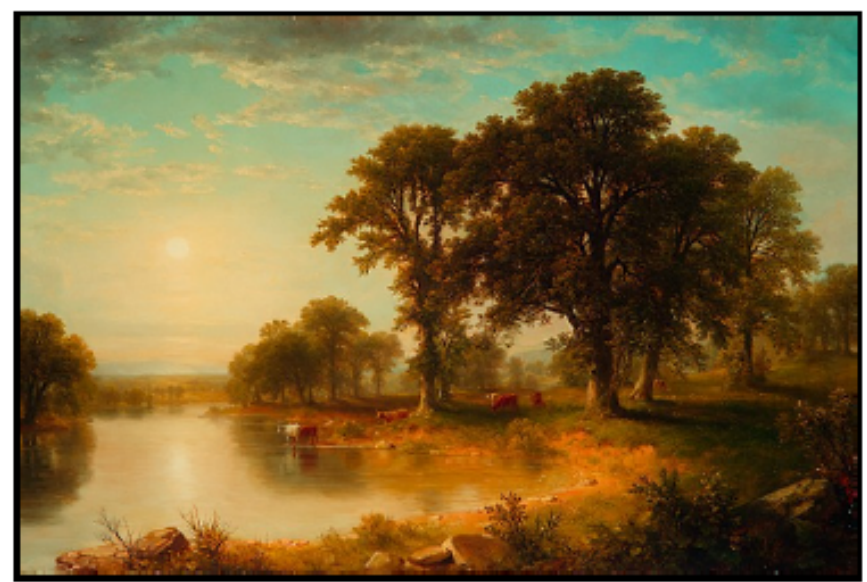

Summer Afternoon

Page 116 


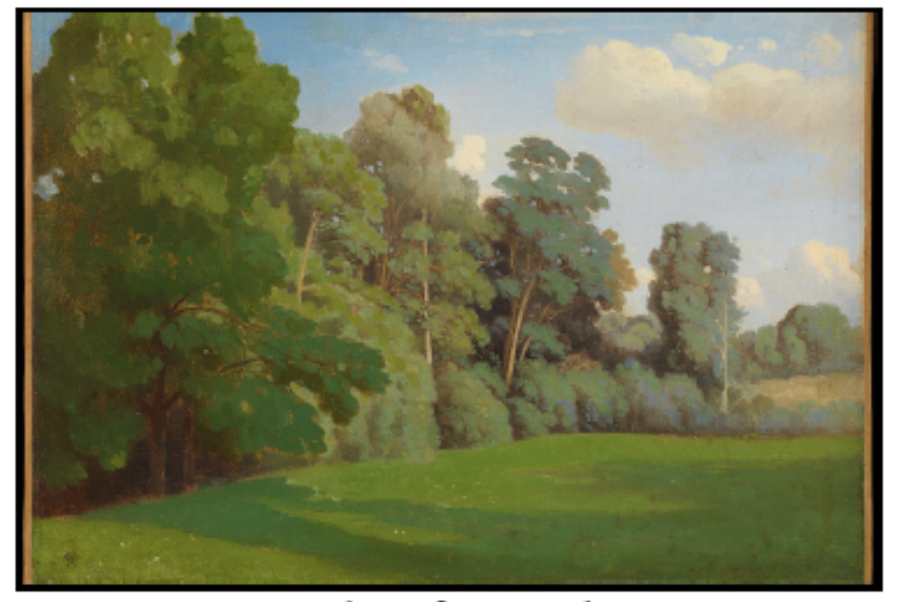

Edge of a Wood

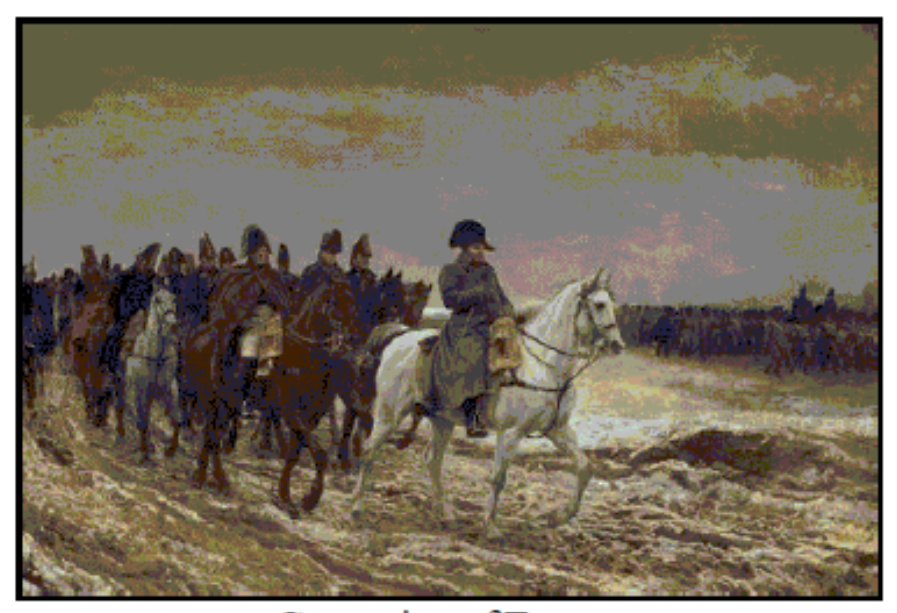

Campaign of France

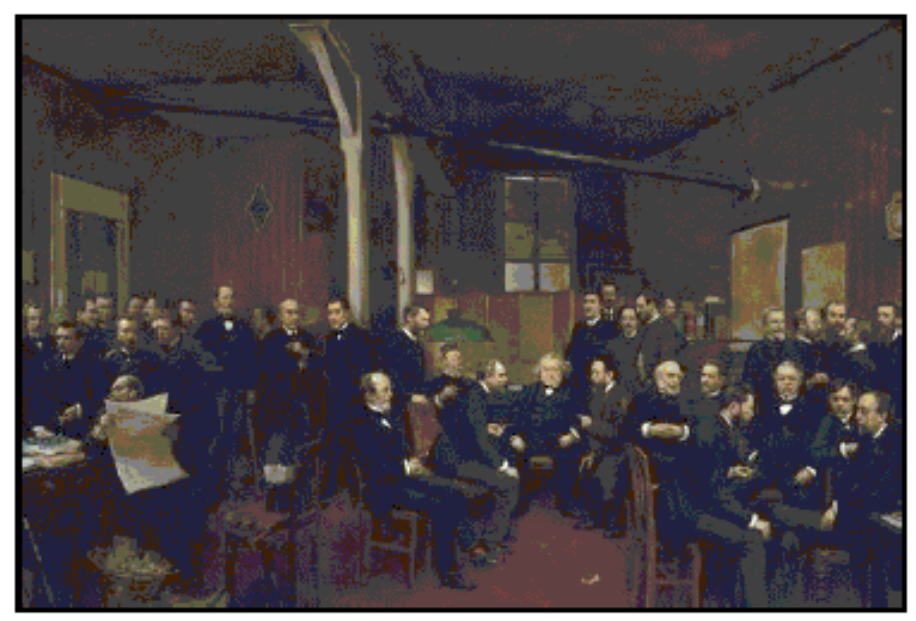

The Newsroom

Page 117 


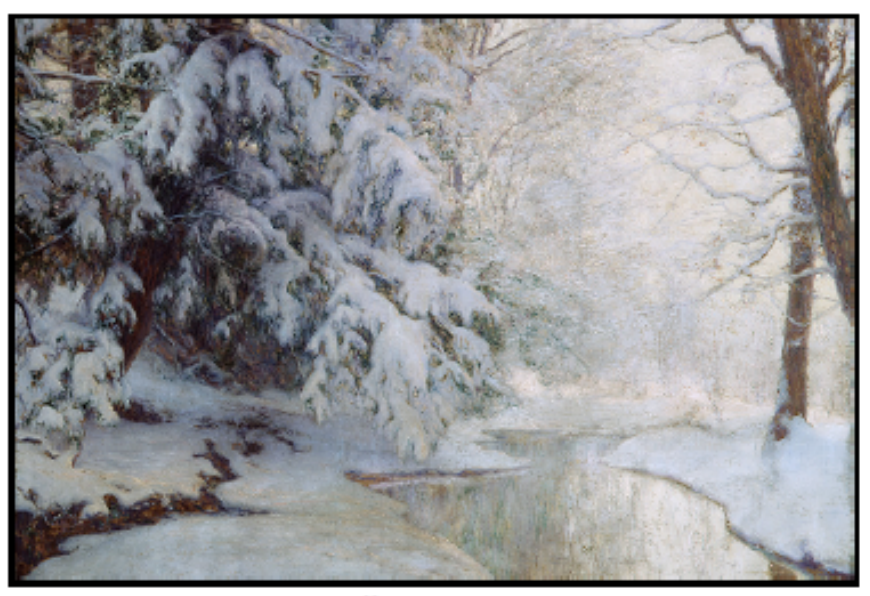

Silent Dawn

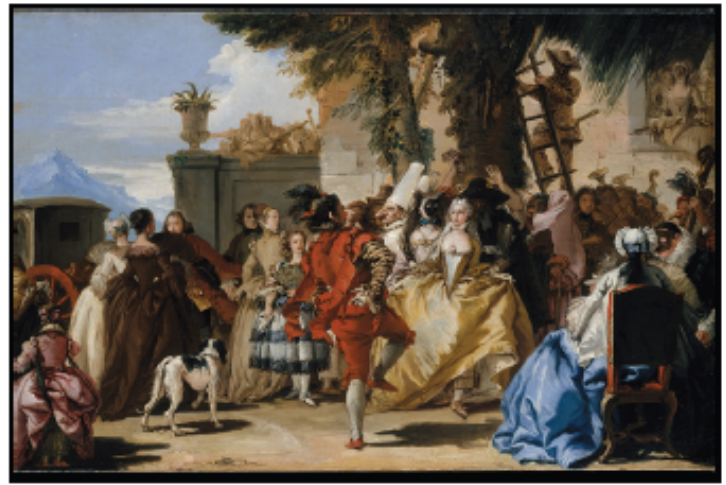

What is the name of the painting above?

Campaign of France

A Dance in the Country

Edge of a Wood

The Newsroom

Silent Dawn

Summer Afternoon

Twilight

Next

Page 118 
What is the setting of the painting Twilight?

Outside on the Water

Outside on a Battle Field

O Outside in a Field or Grassy Area

Inside

Next

What is the primary subject of the painting Summer Afternoon?
Landscape and Animals
- Landscape
O People and Horses
People

Next

How many people are in the painting Edge of a Wood?

At least 1 and at most 5

ONone

More than 10

At least 6 and at most 10

Next 
What is the setting of the painting Campaign of France?

Outside in a Field or Grassy Area

Onside

O Outside on a Battle Field

Outside on the Water

Next

What is the primary subject of the painting The Newsroom?

O People
OLandscape and Animals
O People and Horses
OLandscape

Next

How many people are in the painting Silent Dawn?

At least 6 and at most 10

At least 1 and at most 5

OMore than 10

ONone 


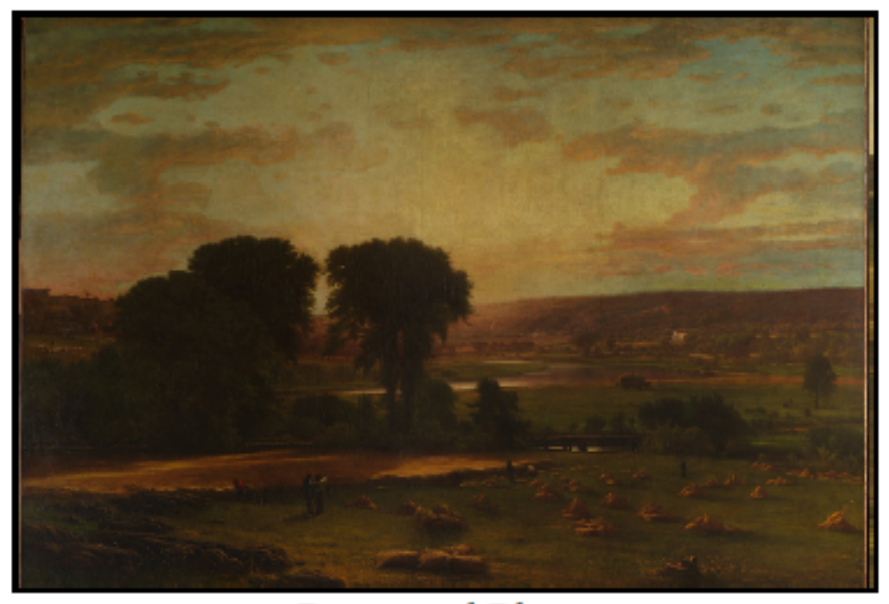

Peace and Plenty

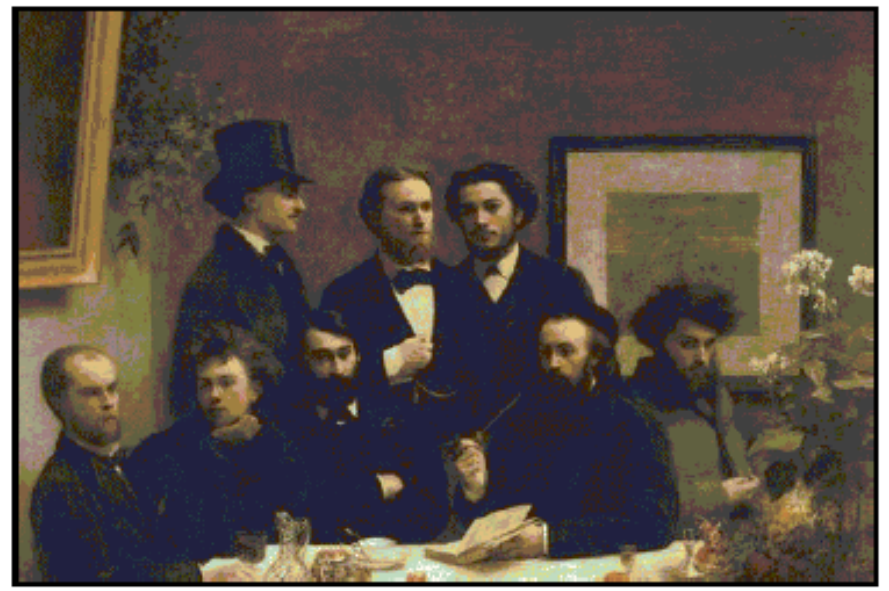

By the Table

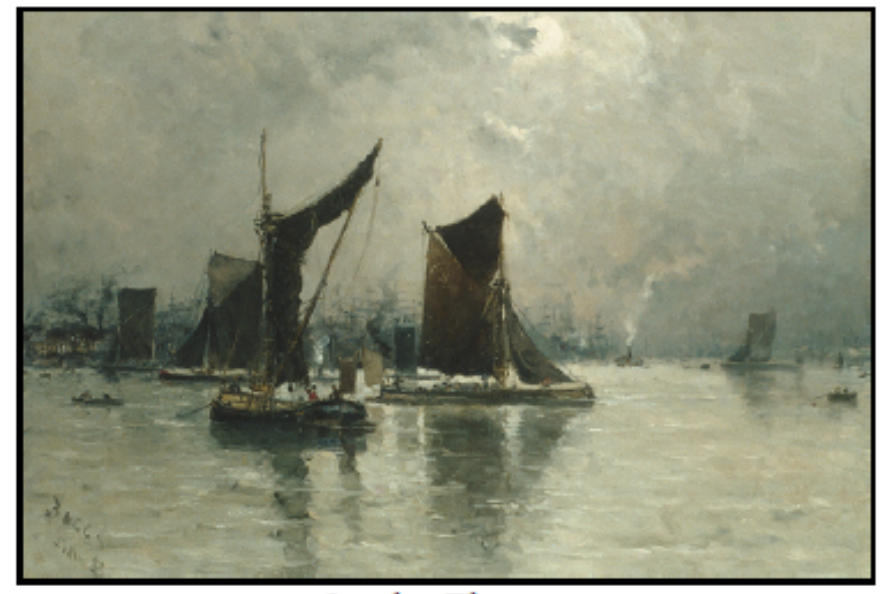

On the Thames

Page 121 


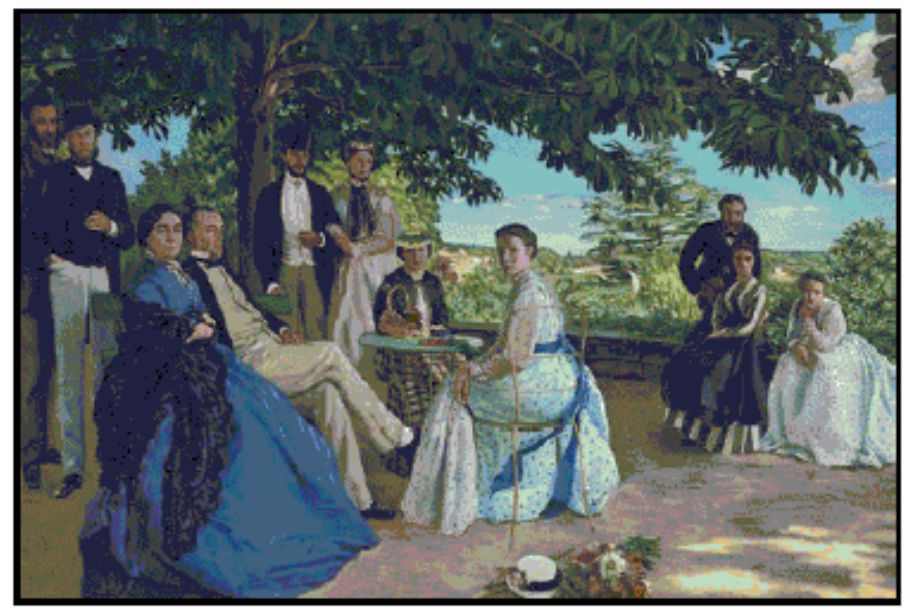

Family Reunion

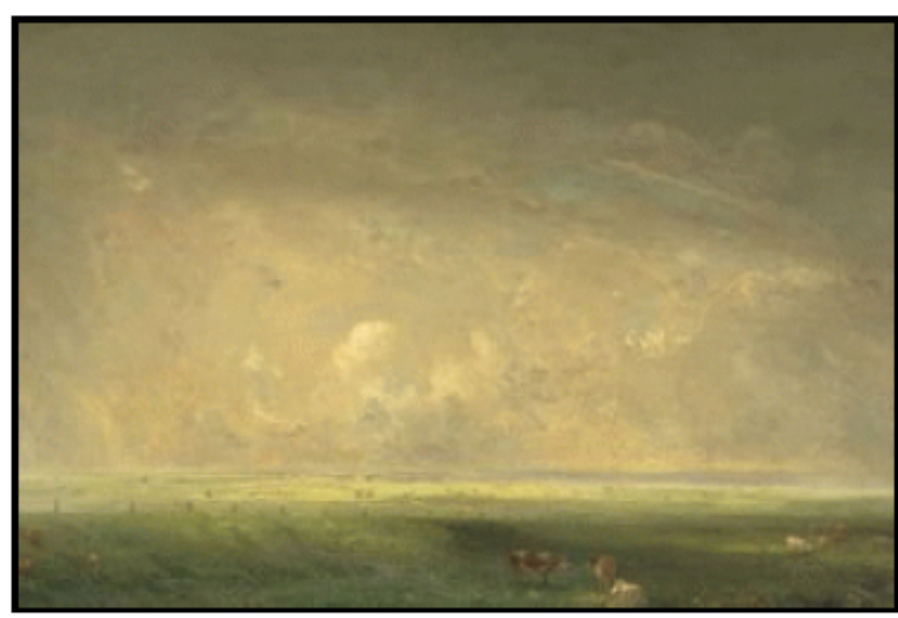

Rain and Sun

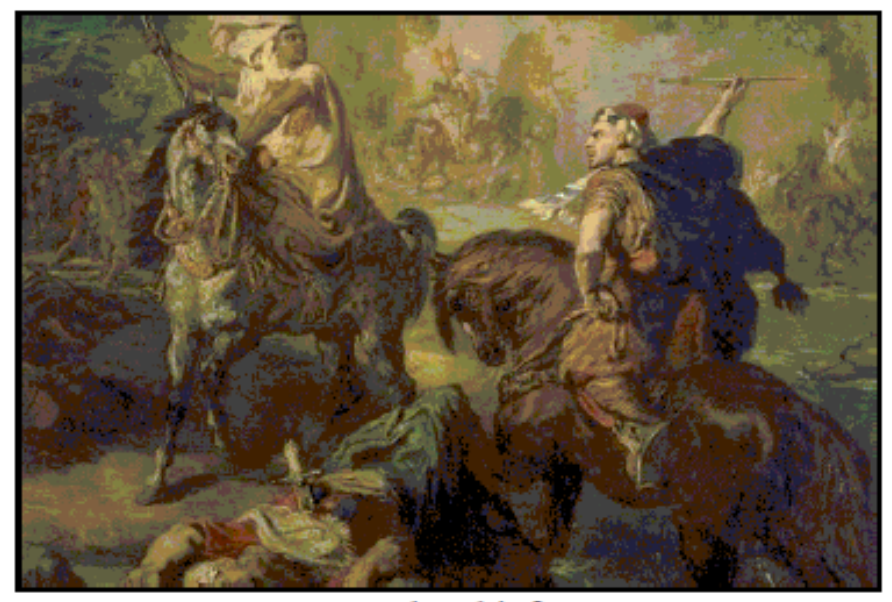

Arab Chiefs

Page 122 


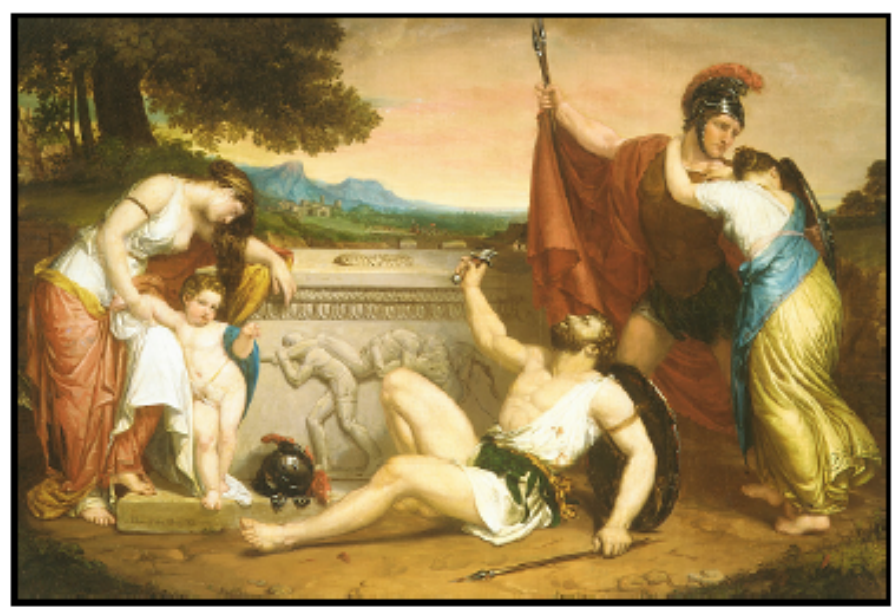

The Wages of War

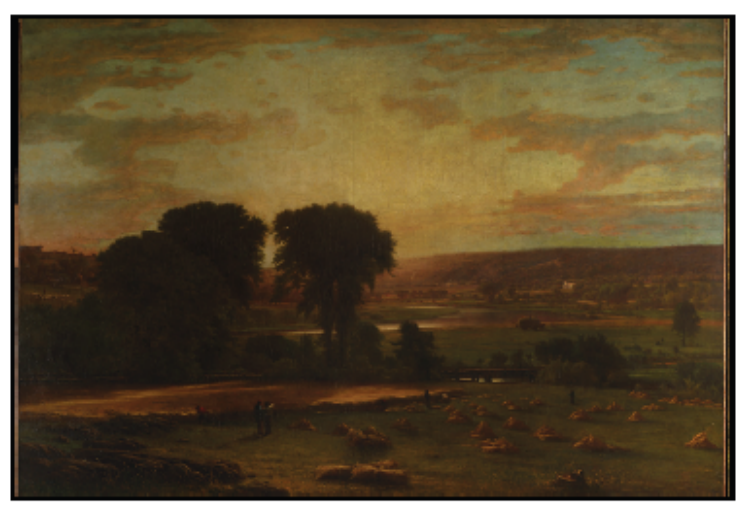

What is the name of the painting above?

\begin{tabular}{|l|}
\hline Arab Chiefs \\
By the Table \\
Family Reunion \\
On the Thames \\
\hline Peace and Plenty \\
\hline Rain and Sun \\
The Wages of War \\
\hline
\end{tabular}

Next

Page 123 
What is the setting of the painting By the Table?

O Inside

Outside on the Water

Outside on a Battle Field

Outside in a Field or Grassy Area

$$
\text { Next }
$$

What is the primary subject of the painting On the Thames?

$$
\begin{aligned}
& \text { People } \\
& \text { Landscape and Animals } \\
& \text { Landscape } \\
& \text { People and Horses }
\end{aligned}
$$

$$
\text { Next }
$$

How many people are in the painting Family Reunion?
C More than 10
None
At least 6 and at most 10
At least 1 and at most 5

$$
\text { Next }
$$

What is the setting of the painting Rain and Sun?

Inside

- Outside in a Field or Grassy Area

Outside on the Water

Outside on a Battle Field

Next

Page 124 
What is the primary subject of the painting Arab Chiefs?

\author{
People \\ Landcape \\ C People and Horses \\ Landscape and Animals
}

Next

How many people are in the painting The Wages of War?

C At least 1 and at most 5

None

More than 10

At least 6 and at most 10

Next

Page 125 
APPENDIX B: Carousel Implementation and Development

The carousels for the experiment and the data collection tools were designed for use on desktop computers through an internet browser. Though Adobe Flash was briefly considered for development of the carousels, the proprietary nature of the code and the need for server-side data collection made this an infeasible development language. A combination of Hypertext Markup Language (HTML), Cascading Style Sheets (CSS), and JavaScript (JS) were used to effectively create the carousels and integrated data collection. The carousels and data collection forms are designed for use in Google Chrome Version 26 on Windows XP, Windows Vista, Windows 7, or Windows 8.

\section{CSS 3}

The ability to develop carousels which are "3D" in nature is possible using CSS 3, the latest working specification for styling web pages. This specification has been developed by the CSS Working Group as a portion of the World Wide Web Consortium (W3C) [1]. Not all browsers support the new features of CSS 3 and many browsers which implement features require browser specific information. A complete list of browser support for various features is available through W3Schools Online Web Tutorials that is updated by W3C [2].

\section{D Transforms}

The specific feature added in CSS 3 that improved the 3D capabilities of the styling language is 3D Transforms. These transformations were previously possible using 2D transforms to effectively scale images for the illusion of a third dimension, but the new feature greatly simplifies any implementation. The transformations include translation, scaling, and rotation on the x-axis, y-axis, and z-axis. The option to use a 
matrix for transformation in a single step is also available. The other expanded feature is the ability to give elements perspective [3].

One of the most accepted and well recognized tutorials for using CSS 3D Transforms was written by David DeSandro [4][5]. The tutorial covers each of the features in the specification with open source examples of static and dynamic interactive styling. Most valuable are the discussion of the geometry and trigonometry that must be used with JS to create a dynamic and adjustable display [6]. This culminates in an example of a carousel of numbered tiles on a flat plane that rotates using a button press. The source code for the carousel presented in the tutorial is the foundation for the carousels that were developed for the experiment [7].

The Modernizr JavaScript library is mentioned in the tutorial and is fundamental in using CSS 3 and other features that have not been full incorporated into published standards and implemented by all browser developers. This library verifies the browser that is in use by a client and creates JavaScript objects and applies CSS properties which are supported by a given browser. Modernizr assists browsers that do not support newer specifications, rendering a feature limited page, rather than failing to load the page. Browsers with the newer specifications render the page with all of the possible features [8].

\section{D Carousel Features}

The original carousel set the background color of each panel using a hue, saturation, and value (HSV) combination across the color spectrum, with a number in the foreground to easily indicate transitions between panels. The carousel also provided 
buttons for the user to change the number of panels on the carousel, rotate the carousel, the axis of rotation, and the visibility of the backface of panels.

\section{Controls}

The first step for the purposes of the experiment was to remove the ability of the user to change the properties of the carousel. The buttons for changing the number of panels, the axis of rotation, and the visibility of the backface of panels were removed. Each of these settings was instead fixed, with 7 panels, a horizontal axis of rotation, and panel backface visibility. The previous and next buttons to rotate the carousel were left for testing and development purposes and centered beneath the main panel. The content of the panels was also changed to display images, removing the numbers from the foreground. CSS allows images to be set as the background rather than setting a specific HSV color [9].

Tilt

The next step was to adjust the axis of rotation so that the carousel rotations were no longer perpendicular to the plane of display. The "tilt" of the carousel magnified the $3 \mathrm{D}$ appearance, further differentiating the design from a $2 \mathrm{D}$ carousel. The original design treated the entire carousel as a single object, placing each panel onto the object and then rotating the object as a whole. This is effective when the rotation is occurring around a single axis. Tilting this entire object would change the orientation of the panels relative to the display so that the panels would appear tilted as well as the carousel.

To prevent the tilt of individual panels, the carousel was redesigned to update the placement of the panels within the carousel on each rotation, rather than simply updating only the carousel object. The function used in initially creating the carousel was called at 
each rotation instead of only for initializing the carousel, which is less efficient but allowed for the panels to move independently. The panels were adjusted in height based on their location relative to the front center panel. Adding this height adjustment to the original rotation creates the illusion of tilting the axis of rotation without in turn tilting the individual panels. The adjustment of height without additional adjustment of the translation towards the center of the carousel creates an ellipse, rather than a circular carousel. This is not visible in the carousels until the tilt is at an angle in excess of $45^{\circ}$. The carousel object as a whole was increased in height to accommodate the size of all of the panels. The border around the centered image was floated further up the screen to be focused around the centered panel.

\section{Backface Visibility}

A side effect of tilting the carousel is that the backface of each panel was readily visible and was the mirror the image which appears on the front of the panel. For the purposes of consistency, the images need to face forward regardless of the orientation of the panel. Using the CSS background-image property would not allow the panels to face forwards while on the back half of the carousel during rotations. The images had to be added directly into the content of the panels and removed from the background. Then the panels are scaled to be flipped on the back half of the carousel so the content of each panel faces the user.

\section{Captions}

Captions for some types of image content needed to be inserted beneath each image. The caption would appear by default on top of the image as in the original carousel with numbering. Increasing the line height and decreasing the text size pushed 
the captions to fit below the images, so the numbers which appeared in the middle of each panel instead appeared below the panels.

\section{Text Areas}

A text area beneath the main image and caption was explored as an option to present additional information for the experiment. Unlike the caption which would be visible for all panels, the text area would remain under the centered image and only display information about that panel. The text area was placed in a separate object below the carousel and adjusted to fit beneath it. A JS array containing the matching text for each panel was indexed and cycled through to fill the text area on each rotation [10]. Unfortunately, with a tilted carousel, the text area covers a portion of the back half of the panels or is displayed far below the carousel requiring the user to scroll. The text area was removed because of this issue and the focus of the experiment remained on the image and captions.

\section{D Carousel Features}

A $2 \mathrm{D}$ carousel to use in comparison to the $3 \mathrm{D}$ carousel was developed using the same combination of HTML, CSS, and JS.

\section{Transitions}

The 2D carousel was initially developed using only HTML and JS. Similar to the text area for the 3D carousel, the content for the 2D carousel could be filled in using JS. When the next or previous button was pressed, the content would "jump" from one panel to another. The panels did not appear to move and the content would change without any visual cue other than the change. The 3D carousel had smooth transitions which took one second to move the entire panels from one location to another [11]. 
The 2D carousel was redeveloped to use the same perspective and set of transitions as the 3D carousel using the same initial model. Instead of using a carousel object, the panels were not rotated but translated across the screen to the left and right of center on the horizontal axis. Each panel would slowly transition from left to right or right to left over the same one second interval as the 3D carousel.

A side effect of the transitions is that as a panel reached the far right edge, it would suddenly transition all the way from the right side of the screen to the left side of the screen, in front of and between other panels. The same side effect would occur on the far left edge of the screen. The user could easily be distracted by this and confused as to the shifting content. Rather than displaying the panel as it moved across the screen, the panels which would be in the back half of the 3D carousel transition out of view. The 2D carousel only displays the panels that would be in the front half of the 3D carousel. The change magnifies the difference between the $2 \mathrm{D}$ and $3 \mathrm{D}$ carousel in addition to correcting the visible edge-to-edge transition.

\section{Scaling}

A 2D carousel did not have an obvious center panel which was larger than any of the other images. The center image of the carousel was adjusted to have a different perspective than the rest of the images in the carousel. The remaining images had to be pushed further to the left and to the right so they would not intersect with the main image. All of the other images were scaled to match the size and relative vertical position of the panels on the 3D carousel which are perpendicular to the screen on the left and right sides of the carousel. 


\section{Experiment Feature Changes}

Once both the 2D and 3D carousels were fully developed in appearance, several changes were made to conform to the final experiment setup design. The number of panels was fixed to seven in total, conforming to the average working memory of a person. The buttons to move between the panels were removed and a single button to start the scrolling or rotation of the carousels was added. The carousel was hidden from view on the initial page load, showing only the button labeled "Start". Once depressed, the carousel would begin scrolling or rotating, changing the center panel once every six seconds [12]. After every panel had been viewed in the center panel for six seconds, the

carousel would fade out of view. These features helped control the environment, allowing equal time for viewing each panel and preventing participants from viewing a panel more than once. A 2D and 3D carousel was created for each set of images of particular content types. With four sets of images per content type, three types of content, and both $2 \mathrm{D}$ and 3D carousels, a total of 24 separate pages were created.

\section{Data Collection}

The ideal situation for collecting data from the carousels was to use forms in the same browser window as the carousel. The participant would be interacting with the same interface and the information about his or her trials would be recorded on the computer. Typically, web forms are used to collect data and store that information onto a server. In this experiment, a web server supporting Active Server Pages (ASP) as well as server side code such as Python or PHP was not available. Instead the data had to be collected on the client computer while still using web forms. 


\section{JavaScript FileWriter Application Programming Interface (API)}

A new API to the JS language is a FileWriter which allows for JS code to create files in a sandboxed filesystem on a portion of a client computer to store information [13]. The API is a working draft, published by the WebApps Working Group on as a portion of W3C [14]. The only browser which has implemented the FileWriter API as an experimental feature and part of its application store is Google Chrome [15].

The most straightforward and complete tutorial of the FileSystem API implementation in Google Chrome was published to the HTML5Rocks Tutorials section and written by Eric Bidelman. The tutorial explains how to gain access to the sandboxed filesystem, interacting with files, creating directories, and moving files[16].

Storing data on the client computer requires several actions on behalf of the client in conjunction with requests through JS code using the FileWriter API. The application settings for Google Chrome must be changed to allow storage space on the computer and to allow file access from webpages. On Windows XP, Windows Vista, Windows 7, and Windows 8 this can be done by adding flags after the program target inside desktop, quick launch, or start menu shortcuts. With these in place, any website can store information on the local computer, a very large security risk. The pages which use this API all show a warning bar at the top asking for the user's permission to store data on his/her local computer. After acceptance for a page, data can be stored for all future sessions until the browser cache is cleared. After clearing, the user will be prompted again.

The JS code first requests for the sandboxed filesystem and temporary or persistent storage space on the local computer. For recording results from an experiment, 
persistent storage which will remain indefinitely unless accessed and deleted by the user is ideal. When the API is used to create a file, the file name including extension is used as a hash into the filesystem to a specific value. The actual file on the local computer is saved without an extension with an eight digit file name. New files are sequentially numbered. Outside of JS code through the original web application, the file names cannot be used to reference or locate the files.

\section{Evaluation Forms}

For each carousel, evaluation forms were created with one question about each panel that was presented in the carousel, in order. Forms used HTML radio buttons and list boxes along with a JS array filled with answers [17]. The questions display one at a time, requiring the user to press next to continue. There is also no means for the user to navigate backwards to revise answers to previous questions. Upon completion of all the questions in an evaluation form, the results are written to a file in the sandboxed filesystem using the current timestamp as the file name to avoid overwriting any data. The carousel information, the trial number, the total number of correct answers, and the individual responses to questions with the corresponding answers are recorded in each file. With the evaluation forms created, the carousels were changed to redirect to the evaluation form after the last panel is displayed, rather than fading away and leaving the user on a blank page.

\section{Personal Information Collection}

A form was also created asking for each participant to enter their first and last name, email address, major, year in school, gender, and age. Upon submission, a new file is created using the FileSystem API storing all of the participant information as well as a 
time stamp. This time stamp is used as a unique identifier for a particular participant through the rest of the forms and for data collection purposes.

\section{Experiment Randomization}

Each participant was expected to view a fully randomized version of the experiment content, with six carousels for training and six carousels for the actual experiment. Within the two sets of trials, subjects were expected to see two carousels containing each type of content, one 2D carousel and one 3D carousel for each type. The order of the six carousels within the training and experiment trials was to be fully randomized. The sets of images were all only to be seen by each participant once throughout the entire experiment. Whether the sets of images were included in the training or experimental trials was also randomized.

Because the assignment of image sets to carousels and carousels to sets of trials both involve ordering within a fixed number of items, a straight random number generator will not adequately select the random ordering. Instead, a random number within a specified range ( 4 for the image sets, 6 for the trial sets) is selected and stored. For the remaining numbers, another random number within the range is generated, checked for uniqueness against all previous numbers generated, stored if it is unique, or regenerated if it is not unique. This process continues until every number within the range has been selected and stored in the order of selection. There is a slight chance that the numbers generated are in order, but this is one possible combination.

In preparation for the randomization, a three dimensional array is filled with the uniform resource locators (URLs) of the 24 carousels. The first dimension corresponds to the content, the second dimension corresponds to the $2 \mathrm{D}$ or $3 \mathrm{D}$ carousel layout, and the 
third dimension specifies the set of images to be displayed within the carousel. Another array is created containing an alternating pattern for selecting a 2D or 3D carousel layout, represented by the values 0 and 1 . Unique arrays of rankings were generated for the three content types and the two sets of trials. Then a set of three arrays are filled with the information about the order, the content option, and the image set for the option.

The final array is filled, using the order as the index into this array, pulling a URL from the three dimensional array indexing first with the content option, second with the 2D or 3D carousel layout, and third with the image set for the option. The list of 12 URLs is inserted as hyperlinks into two separate tables on a page via the document object model DOM, one for the training trials and one for the experiment [18][19].

\section{Qualitative Assessment}

After all of the links have been visited and the carousel evaluations have been performed, a questionnaire about the effectiveness of the display for each carousel in the experiment trials is given to the user. The questionnaire shows the same carousel as displayed during the experiment, with the same six second rotation to remind the user of what they saw. A multidimensional array of the four images sets for the three content types is used to populate the carousel for each question. The styling of the carousel is also modified to match the 2D or 3D layout the participant was originally presented with. Radio buttons are again used to collect the data. Another file is written to the sandboxed file system with the results of this qualitative assessment.

\section{Participant Identification}

In each phase of interaction with a participant data is collected and stored in separate files. As mentioned, the files are named with sequential numbers without the use 
of file extensions and cannot be associated with any particular participant by file name. The timestamp stored with the original personal information is used to act as inter-page communication.

Upon creating the table of hyperlinks, the unique time stamp that identifies the participant is appended onto the end of each URL separated by a hash symbol. The order the carousel appears in a particular trial is also appended after the unique time stamp. The information stored after the hash symbol is known as the window location hash value [20]. When one of the hyperlinks is clicked, the complete URL containing the hash information is opened in a new window or tab. The newly opened page can retrieve the window location hash value from the end of the URL through the DOM.

The carousel pages were modified to take advantage of this property. Although the carousels do not generate files with results, the carousels determine which evaluation is opened and must pass that information on to the evaluation. A carousel retrieves the window location hash value and appends it to the end of the URL for the corresponding evaluation. After the carousel finishes rotating, the evaluation is opened using that URL. The evaluation was also modified to retrieve the window location hash value and to record that at the top of results which are written to the output file in the sandboxed filesystem.

The window location hash is recorded in every data file. The carousel evaluation files also have a record of the order in which they were visited within a trial. Upon review of the data files from the experiment manually or programmatically, the files for the 12 carousels for a single participant and his or her personal information can be brought together in the order they were presented to the participant. 


\section{Fail-safes}

If for any reason the user, browser, or local computer prevent a file from being written using the FileWriter API, a JS alert is used to display the would be contents of the file. The experimenter will be alerted that the data is not being recorded as expected and is presented with the data so it can be recorded manually.

If for any reason a page which writes a data file did not have a hash value, a timestamp is used as a makeshift identification number. The evaluation records an order number of 0 , indicating an order number was not specified in the URL. The files are created sequentially, the participant information, 12 sets of results from carousels, and then the qualitative assessments. When reviewing or aggregating the information after the experiment, the time stamps can be sorted in order to correct the problem and associate a single set of results with a single participant.

\section{Data Aggregation}

A small Java application was written to allow the experimenter to select a folder containing the files generated from any trial runs to create a summary file with the participant information associated to specific results for individual carousels $[21][22][23][24]$. The application relies on a specified format of data files to identify files containing personal information, carousel evaluation results, and the qualitative assessments of the experiment carousels. It also does not assume the files will be accessed sequentially and stores the information into a hash table using the unique identification number as the key for each participant.

After all of the data has been read into internal data structures, the data is written to an external comma separated value sheet. Each row contains the personal information 
of a single participant and the result from a single carousel. The results do not necessarily appear in order because hash tables were also used for the storage of the results from individual carousels.

The original application was designed to read files from only a single directory. However, after the pilot it became clear that the FileWriter API created additional folders and placed data files in separate folders rather than into a single folder. Rather than creating a more complex system of folder selection, the original folder popup was used repeatedly, until the experimenter pressed the cancel button, at which point data was aggregated from all of the folders that were previously selected. 
Carousel Implementation and Development References

1. Bos, Bert, Ed. Home page. Cascading Style Sheets. World Wide Web Consortium, 12 May 2013. Web. 28 December 2012. $<$ http://www.w3.org/Style/CSS/>

2. “CSS3 Browser Support Reference”. W3Schools. Refsnes Data, n.d. Web. 28 December 2012. <http://www.w3schools.com/cssref/css3_browsersupport.asp>

3. “CSS3 3D Transforms". W3Schools. Refsnes Data, n.d. Web. 28 December 2012. <http://www.w3schools.com/css3/css3_3dtransforms.asp>

4. DeSandro, David. Home page. Intro to CSS 3D transforms. GitHub, April 2012. Web. 28 December 2012. <http://desandro.github.io/3dtransforms/>

5. DeSandro, David. "An Introduction to CSS 3-D Transforms". 24-Ways. 24 Ways, 14 December 2010. Web. 28 December 2012. <http://24ways.org/2010/intro-tocss-3d-transforms/>

6. DeSandro, David. "Carousel - Intro to CSS 3D transforms > Docs". Intro to CSS 3D transforms. GitHub, April 2012. Web. 28 December 2012. $<$ http://desandro.github.io/3dtransforms/docs/carousel.html >

7. DeSandro, David. "Carousel 2 - dynamic - Intro to CSS 3D transforms > Docs". Intro to CSS 3D transforms. GitHub, April 2012. Web. 28 December 2012. $<$ http://desandro.github.io/3dtransforms/examples/carousel-02-dynamic.html>

8. Home page. Modernizr. Modernizr, 2 April 2013. Web. 28 December 2012. $<$ http://modernizr.com/> 
9. “CSS Background”. W3Schools. Refsnes Data, n.d. Web. 28 December 2012. <http://www.w3schools.com/css/css_background.asp>

10. “JavaScript Array Object". W3Schools. Refsnes Data, n.d. Web. 28 December 2012.<http://www.w3schools.com/jsref/jsref_obj_array.asp>

11. “CSS3 Transitions”. W3Schools. Refsnes Data, n.d. Web. 28 December 2012. <http://www.w3schools.com/css3/css3_transitions.asp>

12. "Window setTimeout() Method". W3Schools. Refsnes Data, n.d. Web. 5 March 2012. <http://www.w3schools.com/jsref/met_win_settimeout.asp>

13. Uhrhane, Eric, ed. "File Writer API". W3C Standards. W3C, 17 April 2012. Web. 7 April 2013. <http://www.w3.org/TR/file-writer-api/>

14. Lafon, Yves., and Smith, Michael, eds. Home page. W3C Web Apps Working Group. W3C, 25 April 2013. Web. 7 April 2013. <http://www.w3.org/2008/webapps/>

15. “Google Chrome Browser”. W3Schools. Refsnes Data, n.d. Web. 7 April 2013. <http://www.w3schools.com/browsers/browsers_chrome.asp>

16. Bidelman, Eric. "Exploring the FileSystem APIs". HTML5 Rocks Tutorials. HTML5 Rocks, 27 January 2012. Web. 7 April 2013. <http://www.html5rocks.com/en/tutorials/file/filesystem/>

17. “HTML Forms and Input”. W3Schools. Refsnes Data, n.d. Web. 7 April 2013. <http://www.w3schools.com/html/html_forms.asp >

18. “HTML DOM Tutorial”. W3Schools. Refsnes Data, n.d. Web. 7 April 2013. <http://www.w3schools.com/htmldom/> 
19. Document Object Model. W3C, n.d. Web. 7 April 2013. $<$ http://www.w3.org/DOM/>

20. “The Location Object”. W3Schools. Refsnes Data, n.d. Web. 7 April 2013. <http://www.w3schools.com/jsref/obj_location.asp>

21. “Package java.util”. Java Platform Standard Ed. 6. Oracle, n.d. Web. 17 April 2013. <http://docs.oracle.com/javase/6/docs/api/java/util/package-summary.html>

22. "Package java.io". Java Platform Standard Ed. 6. Oracle, n.d. Web. 17 April 2013. <http://docs.oracle.com/javase/6/docs/api/java/io/package-summary.html>

23. "Package javax.swing”. Java Platform Standard Ed. 6. Oracle, n.d. Web. 17 April 2013. <http://docs.oracle.com/javase/6/docs/api/javax/swing/packagesummary.html>

24. "Package javax.swing.filechooser". Java Platform Standard Ed. 6. Oracle, n.d. Web. 17 April 2013. <http://docs.oracle.com/javase/6/docs/api/javax/swing/filechooser/packagesummary.html> 


\section{APPENDIX C: Subject Demographics}

Table 13 Subject Demographics

\begin{tabular}{|c|c|c|c|c|}
\hline $\begin{array}{c}\text { Subject } \\
\#\end{array}$ & Gender & Age & Major & $\begin{array}{c}\text { Experience with 3D Menus on } \\
\text { Computers }\end{array}$ \\
\hline 1 & Female & 20 & Industrial Engineering & Little to no experience \\
\hline 2 & Female & 21 & Industrial Engineering & Little to no experience \\
\hline 3 & Female & 22 & $\begin{array}{c}\text { Liberal Arts and } \\
\text { Engineering Studies }\end{array}$ & Little to no experience \\
\hline 4 & Female & 23 & Mechanical Engineering & Little to no experience \\
\hline 5 & Female & 22 & Mechanical Engineering & Little to no experience \\
\hline 6 & Female & 23 & Industrial Engineering & Little to no experience \\
\hline 7 & Female & 20 & Industrial Engineering & Little to no experience \\
\hline 8 & Female & 24 & Aerospace Engineering & Little to no experience \\
\hline 9 & Female & 21 & Industrial Engineering & Little to no experience \\
\hline 10 & Female & 22 & General Engineering & Little to no experience \\
\hline 11 & Male & 21 & Biomedical Engineering & Little to no experience \\
\hline 12 & Male & 22 & Electrical Engineering & Little to no experience \\
\hline 13 & Male & 21 & Graphic Communications & Little to no experience \\
\hline 14 & Male & 20 & General Engineering & Little to no experience \\
\hline 15 & Male & 21 & Economics & Little to no experience \\
\hline 16 & Male & 19 & Industrial Engineering & Little to no experience \\
\hline 17 & Male & 20 & Architecture & Little to no experience \\
\hline 18 & Male & 18 & Industrial Engineering & Little to no experience \\
\hline 19 & Male & 23 & Architecture & Little to no experience \\
\hline 20 & Male & 21 & Industrial Engineering & Little to no experience \\
\hline
\end{tabular}




\section{APPENDIX D: Experiment Instructions}

Thank you for volunteering to participate in this experiment studying the effects of 2D and 3D visual displays on computers. The duration of this experiment is approximately 30 minutes. For your participation you will receive a $\$ 10$ iTunes gift card.

\section{Instructions:}

Throughout this experiment you will be shown different sets of images.

There are three types of images that you will be shown:

1. Abstract Objects

2. Country Flags

3. Art

These sets of images will be presented either in 2D or 3D, and will each contain seven images. Each image will be shown for a few seconds. After all seven images are presented, seven questions will be asked. There will be a question related to each one of the images in the order that the images were presented.

The table below details the nature of the questions you will be asked for the different types of images:

\begin{tabular}{|l|l|}
\hline \multicolumn{1}{|c|}{ Type of Image } & \multicolumn{1}{c|}{ Nature of Question } \\
\hline Abstract Objects & Location of Object in a 4 Quadrant Grid \\
\hline Flags & $\begin{array}{l}\text { Country Name, Color Scheme, Details on the } \\
\text { Flag }\end{array}$ \\
\hline Art & $\begin{array}{l}\text { Painting Name, Setting, Subject, Number of } \\
\text { People }\end{array}$ \\
\hline
\end{tabular}

Please pay attention to the details of the images, as the purpose of this experiment is to measure your retention of the information presented.

You will first perform six practice trials then will have an opportunity to ask questions. Once questions are answered, you will then proceed to six experimental trials.

Your personal information and individual performance results will remain confidential. At any point during the experiment if you do not wish to continue, you may choose to leave. 


\section{APPENDIX E: Informed Consent Form}

INFORMED CONSENT TO PARTICIPATE IN A RESEARCH PROJECT: The Effect of 2D and 3D Menus on Memory Retention in User Interface Design.

A research project on human computer interaction is being conducted by Angela Muscat in the Department of Industrial and Manufacturing Engineering at Cal Poly, San Luis Obispo The purpose of the study is to determine the effect of 2D and 3D visual displays on retention.

You are being asked to take part in this study by viewing sets of images and answering accompanying questions. Your participation will take approximately thirty minutes. Please be aware that you are not required to participate in this research and you may discontinue your participation at any time without loss of benefits.

There are no possible risks associated with participation in this study. Your confidentiality will be protected; all personal information and your individual performance results will remain confidential.

If you have questions regarding this study or would like to be informed of the results when the study is completed, please feel free to contact Angela Muscat at (206) 940-9197, amuscat@calpoly.edu. If you have questions or concerns regarding the manner in which the study is conducted, you may contact Dr. Steve Davis, Chair of the Cal Poly Human Subjects Committee, at (805) 756-2754, sdavis@ calpoly.edu, or Dr. Dean Wendt, Interim Dean of Research, at (805) 756-1508, dwendt@ calpoly.edu.

If you agree to voluntarily participate in this research project as described, please indicate your agreement by signing below. Please keep one copy of this form for your reference, and thank you for your participation in this research.

Signature of Volunteer

Date

Signature of Researcher

Date 
APPENDIX F: Raw Quantitative Data

Table 14 Raw Quantitative Data

\begin{tabular}{|c|c|c|c|c|c|c|}
\hline & \multicolumn{3}{|c|}{$2 \mathrm{D}$} & \multicolumn{3}{|c|}{$3 \mathrm{D}$} \\
\hline & $\begin{array}{l}\text { Abstract } \\
\text { Objects }\end{array}$ & Flags & Art & $\begin{array}{l}\text { Abstract } \\
\text { Objects }\end{array}$ & Flags & Art \\
\hline \multirow{10}{*}{ Female } & 3 & 4 & 5 & 7 & 5 & 6 \\
\hline & 7 & 2 & 6 & 4 & 3 & 6 \\
\hline & 6 & 3 & 7 & 6 & 4 & 5 \\
\hline & 3 & 2 & 6 & 2 & 4 & 6 \\
\hline & 6 & 1 & 7 & 6 & 1 & 5 \\
\hline & 6 & 3 & 5 & 5 & 4 & 4 \\
\hline & 7 & 6 & 4 & 5 & 5 & 7 \\
\hline & 6 & 7 & 4 & 7 & 4 & 6 \\
\hline & 7 & 5 & 7 & 7 & 4 & 7 \\
\hline & 7 & 5 & 5 & 6 & 3 & 7 \\
\hline \multirow{10}{*}{ Male } & 7 & 5 & 6 & 6 & 7 & 5 \\
\hline & 6 & 2 & 7 & 6 & 4 & 5 \\
\hline & 6 & 3 & 7 & 7 & 4 & 4 \\
\hline & 4 & 6 & 4 & 5 & 3 & 3 \\
\hline & 3 & 7 & 7 & 4 & 5 & 6 \\
\hline & 7 & 3 & 3 & 6 & 3 & 4 \\
\hline & 6 & 4 & 4 & 6 & 6 & 5 \\
\hline & 6 & 4 & 6 & 6 & 3 & 7 \\
\hline & 6 & 2 & 5 & 7 & 5 & 3 \\
\hline & 7 & 5 & 5 & 7 & 6 & 5 \\
\hline
\end{tabular}

Page 146 
APPENDIX G: Raw Questionnaire Data

Table 15 Raw Questionnaire Data

\begin{tabular}{|c|c|c|c|c|c|c|}
\hline & \multicolumn{3}{|c|}{$2 \mathrm{D}$} & \multicolumn{3}{|c|}{$3 \mathrm{D}$} \\
\hline & $\begin{array}{c}\text { Abstract } \\
\text { Objects }\end{array}$ & Flags & Art & $\begin{array}{c}\text { Abstract } \\
\text { Objects }\end{array}$ & Flags & Art \\
\hline \multirow{10}{*}{ Female } & 4 & 4 & 2 & 4 & 4 & 4 \\
\hline & 1 & 1 & 3 & 4 & 3 & 5 \\
\hline & 3 & 3 & 3 & 4 & 4 & 4 \\
\hline & 2 & 3 & 4 & 4 & 1 & 3 \\
\hline & 4 & 4 & 4 & 4 & 4 & 4 \\
\hline & 5 & 3 & 5 & 3 & 1 & 2 \\
\hline & 3 & 2 & 5 & 4 & 4 & 5 \\
\hline & 4 & 4 & 4 & 2 & 2 & 2 \\
\hline & 4 & 5 & 5 & 5 & 4 & 4 \\
\hline & 4 & 2 & 1 & 4 & 4 & 4 \\
\hline \multirow{10}{*}{ Male } & 5 & 5 & 5 & 5 & 5 & 5 \\
\hline & 3 & 3 & 3 & 4 & 4 & 4 \\
\hline & 4 & 4 & 4 & 3 & 3 & 3 \\
\hline & 4 & 3 & 4 & 4 & 3 & 4 \\
\hline & 4 & 3 & 3 & 1 & 4 & 5 \\
\hline & 3 & 3 & 2 & 5 & 4 & 4 \\
\hline & 3 & 2 & 3 & 4 & 3 & 4 \\
\hline & 5 & 1 & 3 & 5 & 3 & 3 \\
\hline & 3 & 1 & 2 & 4 & 3 & 4 \\
\hline & 2 & 2 & 2 & 4 & 4 & 4 \\
\hline
\end{tabular}

Page 147 
APPENDIX H: Subjects' Comments

Table 16 Subjects' Comments from Questionnaire

\begin{tabular}{|c|c|}
\hline $\begin{array}{l}\text { Subject } \\
\#\end{array}$ & Comments \\
\hline 1 & No comments \\
\hline 2 & $\begin{array}{l}\text { I was able to identify people and places in a 3D model the best. I had a lot } \\
\text { of difficulty with associating colors and symbols on flags with the name } \\
\text { of the country, both with 2D and 3D. Overall I would prefer 3D due to it } \\
\text { allowing you more time to remember and to repeat information. }\end{array}$ \\
\hline 3 & $3 \mathrm{D}$ was easier to remember than $2 \mathrm{D}$ \\
\hline 4 & $\begin{array}{l}\text { For the abstract images where we were asked to recall the quadrants, it } \\
\text { helped to see the 3D version because I could keep reminding myself of } \\
\text { the quadrant and thus create a sequence in my mind to remember the } \\
\text { quadrants. I felt as though the fact that the 3D display rotated the images } \\
\text { so that the viewer was actually seeing the back of the image was } \\
\text { confusing and did not help with a quick reminder of what the image was. } \\
\text { When there wasn't a sequence involved (the paintings and flags) I felt I } \\
\text { could focus more on the individual image being presented when it was the } \\
\text { 2D linear sequence. }\end{array}$ \\
\hline 5 & $\begin{array}{l}\text { I was able to remember more of the } 3 \mathrm{D} \text { images than the } 2 \mathrm{D} \text { images } \\
\text { shown. }\end{array}$ \\
\hline 6 & $\begin{array}{l}\text { I preferred the } 2 \mathrm{D} \text { displays rather than the } 3 \mathrm{D} \text { mainly because I was able } \\
\text { to see the previous slide and name while also looking at the current slide. }\end{array}$ \\
\hline 7 & $\begin{array}{l}\text { I think the 3D displays helped me retain more information because during } \\
\text { the rotation of the images, I could go back and compare different slides in } \\
\text { my mind. This made it easier to remember the content. Retaining the } \\
\text { images of the flags was very difficult because they are so similar to each } \\
\text { other, but even in this case, I found the 3D to be a better display. }\end{array}$ \\
\hline 8 & $\begin{array}{l}\text { A main difference between the } 2 \mathrm{D} \text { and the } 3 \mathrm{D} \text { was that the images for the } \\
\text { 3D were in continual view (but at some times details were unreadable or } \\
\text { blurred). It was distracting to have the images rotate behind the one that } \\
\text { you're trying to focus and remember. The 2D had the advantage of } \\
\text { focusing on the image, and briefing referring back to the previous or a } \\
\text { quick sneak peak on the upcoming one. The 2D carousel was set up in a } \\
\text { more linear fashion, which lends itself to be less distracting (for me). The } \\
\text { 3D carousel lends itself to more exposure of the image to the individual } \\
\text { but a lesser level of retention (for me..). It seems as if a 3D screen lends } \\
\text { itself better to quick reference to be used for brief quick selections; } \\
\text { whereas if you need to actually focus or pay attention to something the } \\
\text { 2D seems to be more suited for the job. Granted-- everything is usually } \\
\text { presented in a linear fashion, so maybe that's why a 2D image lends itself } \\
\text { to be more 'intuitive' for the user. }\end{array}$ \\
\hline
\end{tabular}




\begin{tabular}{|c|c|}
\hline 9 & $\begin{array}{l}\text { The } 3 \text {-D displays were distracting, I would try to look at all of them at } \\
\text { once to anticipate the next image, while the 2-D display only allowed me } \\
\text { to look one ahead and not get too distracted. }\end{array}$ \\
\hline 10 & $\begin{array}{l}\text { The 3D display was more effective because it allowed me to review the } \\
\text { previous images shown if I felt insecure about my memory retention in } \\
\text { any way. This mainly helped with the names of images rather than what } \\
\text { was contained in the images. 3D display was superior for memory } \\
\text { retention in relation to the flags and paintings but the } 2 \mathrm{D} \text { and } 3 \mathrm{D} \text { displays } \\
\text { seemed to have the same effect on memory retention for the quadrant } \\
\text { questions. }\end{array}$ \\
\hline 11 & $\begin{array}{l}\text { 3D display makes it easier to retain the images because it captivated me } \\
\text { more. On the } 2 \mathrm{D} \text { display I felt like a blanked out more. }\end{array}$ \\
\hline 12 & $2 \mathrm{~d}$ presentation in the shape of a wheel would be best. \\
\hline 13 & $\begin{array}{l}\text { The flags in general were hard. The 3D flags made it harder. I caught } \\
\text { myself trying to look back a few flags to try to remember, then forgot } \\
\text { what the flag I was on was. }\end{array}$ \\
\hline 14 & $\begin{array}{l}\text { 2D felt more capable of delivering a lot of information about a single } \\
\text { picture or flag while 3D did a better job of showing the general } \\
\text { characteristics of each image. }\end{array}$ \\
\hline 15 & $\begin{array}{l}\text { The shapes in quadrants was very diffcult and confusing in a 3D form. I } \\
\text { wasn't able to tell if the shapes were mirrored or I was seeing them from } \\
\text { the back as if they were transparent. }\end{array}$ \\
\hline 16 & $\begin{array}{l}\text { With 3D displays, I am able to see all the other task displays in the } \\
\text { background. }\end{array}$ \\
\hline 17 & $\begin{array}{l}\text { I'm not sure if I noticed much of a change in my mental processes with } \\
\text { the } 2 \mathrm{~d} \text { vs the } 3 \mathrm{~d} \text { displays. What helped me a little with the } 3 \mathrm{~d} \text { was that I } \\
\text { was able to look at all of the past pictures instead of just the three shown } \\
\text { for the } 2 \mathrm{~d} \text { display. }\end{array}$ \\
\hline 18 & $\begin{array}{l}\text { I thought the 3D displays were definitely more helpful than the 2D } \\
\text { displays. Although there was more information on the screen in the 3D } \\
\text { displays I was still able to retain more information than with the 2D } \\
\text { displays. }\end{array}$ \\
\hline 19 & $\begin{array}{l}\text { arrays in three dimensions seem to allow for more information on the } \\
\text { screen while retaining a hierarchy that allows focus on certain } \\
\text { information }\end{array}$ \\
\hline 20 & $\begin{array}{l}\text { Wanted to point out how with the } 3 \mathrm{D} \text { displays I could still look at every } \\
\text { picture, I think that contributed to my retention. }\end{array}$ \\
\hline
\end{tabular}

